\title{
Quantum Symmetries of the Twisted Tensor Products of $\mathrm{C}^{*}$-Algebras
}

\author{
Jyotishman Bhowmick ${ }^{1}$, Arnab Mandal ${ }^{2}$, Sutanu Roy ${ }^{2}$, Adam Skalski ${ }^{3}$ \\ ${ }^{1}$ Statistics and Mathematics Unit, Indian Statistical Institute, 203, B. T. Road, Kolkata 700108, India. \\ E-mail: jyotishmanb@gmail.com \\ 2 School of Mathematical Sciences, National Institute of Science Education and Research Bhubaneswar, \\ HBNI, Jatni 752050, India. E-mails: arnab@niser.ac.in; sutanu@ niser.ac.in \\ 3 Institute of Mathematics of the Polish Academy of Sciences, ul. Sniadeckich 8, 00-656 Warsaw, Poland. \\ E-mail: a.skalski@impan.pl
}

Received: 17 April 2018 / Accepted: 31 August 2018

Published online: 13 October 2018 - () The Author(s) 2018

\begin{abstract}
We consider the construction of twisted tensor products in the category of $\mathrm{C}^{*}$-algebras equipped with orthogonal filtrations and under certain assumptions on the form of the twist compute the corresponding quantum symmetry group, which turns out to be the generalized Drinfeld double of the quantum symmetry groups of the original filtrations. We show how these results apply to a wide class of crossed products of $C^{*}$ algebras by actions of discrete groups. We also discuss an example where the hypothesis of our main theorem is not satisfied and the quantum symmetry group is not a generalized Drinfeld double.
\end{abstract}

\section{Introduction}

The study of quantum symmetry groups (in the framework of compact quantum groups of Woronowicz [48]) has started from the seminal paper of Wang [46], who studied quantum permutation groups and quantum symmetry groups of finite-dimensional $\mathrm{C}^{*}$-algebras equipped with reference states. Soon after this, the theory of quantum symmetries was extended to finite metric spaces and finite graphs by Banica, Bichon and their collaborators (see $[3,4,13]$, and more recently $[23,41]$ ), who uncovered several interesting connections to combinatorics, representation theory and free probability $([5,37,43]$ and the references therein). The next breakthrough came through the work of Goswami and his coauthors $[9,20]$, who introduced the concept of quantum isometry groups associated to a given spectral triple á la Connes, viewed as a noncommutative differential manifold (for a general description of Goswami's theory we refer to a recent book [21], another introduction to the subject of quantum symmetry groups may be found in the lecture notes [1]). Among examples fitting in the Goswami's framework were the spectral triples associated with the group $\mathrm{C}^{*}$-algebras of discrete groups, whose quantum isometry groups were first studied in [12], and later analyzed for example in $[6,7,31]$.

Historically, the main source of examples of quantum groups was the deformation theory related to the quantum method of the inverse problem and the desire to study 
a quantum version of the Yang-Baxter equation [45]; and in fact already this early work gave rise to the construction of what is now called the Drinfeld double, which plays an important role in this paper. Later, when the theory became to be viewed as one of the instances of the noncommutative mathematics à la Connes [16], there was a hope that by analogy with the classical situation the quantum groups might arise as quantum symmetries of physical objects appearing in the quantum field theory. It is worth mentioning that Goswami's theory was in particular applied to compute the quantum isometry group of the finite spectral triple corresponding to the standard model in particle physics $[15,16]$, for which we refer to Chapter 9 of [21].

In fact the examples related to group $C^{*}$-algebras of discrete groups motivated Banica and Skalski to introduce in [8] a new framework of quantum symmetry groups based on orthogonal filtrations of unital $\mathrm{C}^{*}$-algebras, which will be the main focus of our paper. Before we pass to a more specific description, we should mention that Thibault de Chanvalon generalized in [18] this approach further to orthogonal filtrations of Hilbert $\mathrm{C}^{*}$-modules. The concept of an orthogonal filtration of a given unital $\mathrm{C}^{*}$-algebra $A$ with a reference state $\tau_{A}$ is essentially a family of mutually orthogonal (with respect to the scalar product coming from $\tau_{A}$ ) finite-dimensional subspaces spanning a dense subspace of $A$. The corresponding quantum symmetry group is the universal compact quantum group acting on $A$ in such a way that the individual subspaces are preserved. The article [8] proves that such a universal action always exists and discusses several examples. The problems related to the study of quantum symmetry groups in this setup are two-fold: first we need to construct a natural filtration on a $\mathrm{C}^{*}$-algebra, and then we want to compute the corresponding quantum symmetry group.

The starting point for this work was an observation that if a $\mathrm{C}^{*}$-algebra $A$ is equipped with an orthogonal filtration and we have an action of a discrete group $\Gamma$ on $A$, preserving the state $\tau_{A}$, then the corresponding crossed product admits a natural orthogonal filtration (Proposition 6.13), which we will denote $\widetilde{A} \rtimes_{\beta} \widetilde{B}$. Note here that the crossed product construction, generalizing that of a group $C^{*}$-algebra, on one hand yields a very rich and intensely studied source of examples of operator algebras, and on the other was originally motivated by the desire to model inside the same $\mathrm{C}^{*}$-algebra both the initial system, and the group acting on it in a way compatible with the action-in other words, making the action implemented by a unitary representation. The attempts to compute and analyze the resulting quantum symmetry groups have led, perhaps unexpectedly, to discovering deep connections between the quantum symmetry group construction and the notion of a generalized Drinfeld double of a pair of (locally) compact quantum groups linked through a bicharacter, as studied for example in $[2,39]$. This motivated us to extend the original question to the context of twisted tensor products of [35].

Let $A$ and $B$ be $C^{*}$-algebras equipped with reduced actions $\gamma_{A}$ and $\gamma_{B}$ of locally compact quantum groups $G$ and $H$, respectively. Then the twisted tensor product of $A$ and $B$, denoted $A \otimes_{\mathrm{V}_{1}} B$, is a $C^{*}$-algebra defined in terms of the maps $\gamma_{A}, \gamma_{B}$ and a bicharacter $\mathrm{V}_{1}$ belonging to the unitary multiplier algebra of $\mathrm{C}_{0}(\hat{G}) \otimes \mathrm{C}_{0}(\hat{H})$. For the trivial bicharacter $\mathrm{V}_{1}=1$, the $\mathrm{C}^{*}$-algebra $A \otimes_{\mathrm{V}_{1}} B$ is the minimal tensor product $A \otimes B$. When $A, B$ are unital $C^{*}$-algebras, $A \nabla_{\mathrm{V}_{1}} B$ is a unital $\mathrm{C}^{*}$-algebra.

Our main result is the following: suppose that $A$ and $B$ are two unital $C^{*}$-algebras equipped with orthogonal filtrations $\widetilde{A}$ and $\widetilde{B}$, respectively, that $\gamma_{A}, \gamma_{B}$ are filtration preserving reduced actions of compact quantum groups $G$ and $H$ on $A$ and $B$, and that $\mathrm{V}_{1} \in \mathcal{U}\left(\mathrm{C}_{0}(\hat{G}) \otimes \mathrm{C}_{0}(\hat{H})\right)$ is a bicharacter. Universality of $\operatorname{QISO}(\widetilde{A})$ and $\operatorname{QISO}(\widetilde{B})$ gives a unique bicharacter $\mathrm{V} \in \mathcal{U}\left(\mathrm{C}_{0}(\widehat{\mathrm{QISO}(\widetilde{A})}) \otimes \mathrm{C}_{0}(\widehat{\mathrm{QISO}(\widetilde{B})})\right)$ lifting $\mathrm{V}_{1}$. Then one can always construct a natural orthogonal filtration $\widetilde{A} \nabla_{\mathrm{V}_{1}} \widetilde{B}$ on $A \nabla_{\mathrm{V}_{1}} B$, and moreover, the 
resulting quantum symmetry group $\operatorname{QISO}\left(\widetilde{A} \otimes_{\mathrm{V}_{1}} \widetilde{B}\right)$ is a generalized Drinfeld double of $\operatorname{QISO}(\widetilde{A})$ and $\operatorname{QISO}(\widetilde{B})$ with respect to $\mathrm{V}$ (Theorems 5.1 and 5.4).

Next, we try to apply Theorem 5.4 to compute the quantum symmetry group of $A \rtimes_{\beta, \mathrm{r}} \Gamma$ in terms of the quantum symmetry groups of $\widetilde{A}$ and $\widetilde{B}$, where the latter is a natural filtration of $\mathrm{C}_{\mathrm{r}}^{*}(\Gamma)$. It turns out (Theorem 6.2) that if the action $\beta$ of $\Gamma$ on $A$ factors through the action of the quantum symmetry group of $\widetilde{A}$ then indeed, we can apply Theorem 5.1 to prove that the quantum symmetry group of $A \rtimes_{\beta, \mathrm{r}} \Gamma$ is a generalized Drinfeld double of the quantum symmetry groups of $\widetilde{A}$ and $\widetilde{B}$. Our result can be applied to a wide class of crossed products, including noncommutative torus, the Bunce-Deddens algebra, crossed products of Cuntz algebras studied by Katsura as well as certain crossed products related to compact quantum groups and their homogeneous spaces. We also exhibit an example where the hypothesis of Theorem 6.2 does not hold and the quantum symmetry group of the crossed product is not of the generalized Drinfeld double form.

In order to establish Theorem 5.4, we need a certain universal property of the universal $\mathrm{C}^{*}$-algebra associated with a Drinfeld double. Although in the context of quantum symmetry groups it suffices to work with compact/discrete quantum groups, the property we mention remains true in the general locally compact setting, being a natural framework for studying twisted tensor products and Drinfeld doubles; thus we choose to consider this level of generality in the first few sections of the paper. For locally compact quantum groups, we refer to $[27,28,47]$; we will in fact only use the $C^{*}$-algebraic aspects of the theory.

The plan of the article is as follows: in Sect. 2, after fixing the notations and conventions for locally compact quantum groups, we discuss the theories of twisted tensor product of $\mathrm{C}^{*}$-algebras and generalized Drinfeld doubles developed in [35,39], respectively. In Sect. 3 we study the 'universal $C^{*}$-algebra' associated to the generalized Drinfeld double, and prove two results about its action on twisted tensor products, namely Lemma 3.4 and Theorem 3.5. Section 4 is devoted to showing that if $A$ and $B$ are unital $\mathrm{C}^{*}$-algebras equipped with orthogonal filtrations and $\mathrm{V}$ is a bicharacter in the unitary multiplier algebra of $\mathrm{C}_{0}(\widehat{\mathrm{QISO}(\widetilde{A})}) \otimes \mathrm{C}_{0}(\widehat{\mathrm{QISO}(\widetilde{B})})$, then the twisted tensor product $A \bigotimes_{\mathrm{V}} B$ also admits a natural orthogonal filtration $\widetilde{A} \bigotimes_{\mathrm{V}} \widetilde{B}$ with respect to the twisted tensor product state. In Sect. 5 we prove the main result of this article which says that $\operatorname{QISO}\left(\widetilde{A} \otimes_{\mathrm{V}} \widetilde{B}\right)$ is canonically isomorphic to the generalized Drinfeld double of QISO $(\widetilde{A})$ and QISO $(\widetilde{B})$ with respect to the bicharacter V. Finally in Sect. 6 we show that the above theorem applies to reduced crossed products $A \rtimes_{\beta, r} \Gamma$ for group actions of a specific form (Theorem 6.2). We also discuss several natural examples in which the assumptions of Theorem 6.2 are satisfied. In Sect. 6.4, we show that the conclusion of Theorem 6.2 fails to hold for more general actions. Finally we discuss further possible extensions of such a framework to twisted crossed products and to crossed products by actions of discrete quantum groups.

\section{Preliminaries}

Let us fix some notations and conventions. For a normed linear space $A, A^{\prime}$ will denote the set of all bounded linear functionals on $A$. All Hilbert spaces and $\mathrm{C}^{*}$-algebras (which are not explicitly multiplier algebras) are assumed to be separable. For a $\mathrm{C}^{*}$-algebra $A$, let $\mathcal{M}(A)$ be its multiplier algebra and let $\mathcal{U}(A)$ be the group of unitary multipliers of $A$. For two norm closed subsets $X$ and $Y$ of a $C^{*}$-algebra $A$ and $T \in \mathcal{M}(A)$, let

$$
X T Y:=\{x T y \mid x \in X, y \in T\}^{\mathrm{CLS}}
$$


where CLS stands for the closed linear span.

Let $\mathfrak{C}^{*} \mathfrak{a l g}$ be the category of $\mathrm{C}^{*}$-algebras with nondegenerate *-homomorphisms $\pi: A \rightarrow \mathcal{M}(B)$ as morphisms from $A$ to $B$ (with the composition understood via strict extensions). Moreover, $\operatorname{Mor}(A, B)$ will denote this set of morphisms.

Let $\mathcal{H}$ be a Hilbert space. A representation of a $C^{*}$-algebra $A$ on $\mathcal{H}$ is a nondegenerate *-homomorphism $\pi: A \rightarrow \mathbb{B}(\mathcal{H})$. Since $\mathbb{B}(\mathcal{H})=\mathcal{M}(\mathbb{K}(\mathcal{H})$ ) (where $\mathbb{K}(\mathcal{H})$ denotes the algebra of compact operators on $\mathcal{H})$ and the nondegeneracy conditions $A \mathbb{K}(\mathcal{H})=\mathbb{K}(\mathcal{H})$ and $A \mathcal{H}=\mathcal{H}$ are equivalent, we have $\pi \in \operatorname{Mor}(A, \mathbb{K}(\mathcal{H}))$.

We write $\Sigma$ for the tensor flip $\mathcal{H} \otimes \mathcal{K} \rightarrow \mathcal{K} \otimes \mathcal{H}, x \otimes y \mapsto y \otimes x$, for two Hilbert spaces $\mathcal{H}$ and $\mathcal{K}$. We write $\sigma$ for the tensor flip isomorphism $A \otimes B \rightarrow B \otimes A$ for two $C^{*}$-algebras $A$ and $B$. Further we use the standard 'leg' notation for maps acting on tensor products.

\subsection{Quantum groups.}

Definition 2.1. A Hopf $\mathrm{C}^{*}$-algebra is a pair $\left(C, \Delta_{C}\right)$ consisting of a $\mathrm{C}^{*}$-algebra $C$ and an element $\Delta \in \operatorname{Mor}(C, C \otimes C)$ such that

(1) $\Delta_{C}$ is coassociative: $\left(\Delta_{C} \otimes \mathrm{id}_{C}\right) \circ \Delta_{C}=\left(\mathrm{id}_{C} \otimes \Delta_{C}\right) \circ \Delta_{C}$;

(2) $\Delta_{C}$ satisfies the cancellation conditions: $\Delta_{C}(C)\left(1_{C} \otimes C\right)=C \otimes C=(C \otimes$ $\left.1_{C}\right) \Delta_{C}(C)$.

Let $\left(D, \Delta_{D}\right)$ be a Hopf $C^{*}$-algebra. A Hopf ${ }^{*}$-homomorphism from $C$ to $D$ is an element $f \in \operatorname{Mor}(C, D)$ such that $(f \otimes f)\left(\Delta_{C}(c)\right)=\Delta_{D}(f(c))$ for all $c \in C$.

A compact quantum group or CQG, in short, is described by a Hopf $\mathrm{C}^{*}$-algebra $\left(C, \Delta_{C}\right)$ such that $C$ is unital (in which case $C$ is often called a Woronowicz algebra). Hopf *-homomorphisms between CQGs are called CQG morphisms.

Example 2.2. Every compact group $G$ can be viewed as a compact quantum group by setting $C=\mathrm{C}(G)$ and $\left(\Delta_{C} f\right)\left(g_{1}, g_{2}\right):=f\left(g_{1} g_{2}\right)$ for all $f \in \mathrm{C}(G), g_{1}, g_{2} \in G$. Also, every discrete group $\Gamma$ gives rise to a compact quantum group by fixing $C=\mathrm{C}_{\mathrm{r}}^{*}(\Gamma)$ and $\Delta_{C}\left(\lambda_{g}\right):=\lambda_{g} \otimes \lambda_{g}$, where $\lambda$ is the regular representation of $\Gamma$ on $L^{2}(\Gamma)$ and $g \in \Gamma$ is arbitrary; we could as well choose here the full group $C^{*}$-algebra $C^{*}(\Gamma)$, as will be discussed below.

Nonunital Hopf $\mathrm{C}^{*}$-algebras are noncommutative analogue of locally compact semigroups satisfying the cancellation property. The question of how one should define locally compact quantum groups was studied for many years, with the approach by multiplicative unitaries initiated by Baaj and Skandalis [2] and later developed in [47] by Woronowicz, and a generally accepted notion based on von Neumann algebraic techniques proposed by Kustermans and Vaes [29] (see also a later paper [33]). One should note here also an earlier von Neumann algebraic approach presented in [32], and also the fact that both articles [33] and [29] drew on the algebraic duality techniques of [44].

Thus a locally compact quantum group $G$ is a virtual object studied via its associated operator algebras, in particular the von Neumann algebra $\mathrm{L}^{\infty}(G)$ equipped with a comultiplication $\Delta: \mathrm{L}^{\infty}(G) \rightarrow \mathrm{L}^{\infty}(G) \bar{\otimes} \mathrm{L}^{\infty}(G)$ and the left and right Haar weights. We assume that $\mathrm{L}^{\infty}(G)$ is represented on $\mathrm{L}^{2}(G)$, the GNS-Hilbert space of the right Haar weight. The key fact connecting the approach of Kustermans and Vaes with these of $[2,47]$ is the existence of a distinguished unitary $\mathrm{W}^{G} \in \mathcal{U}\left(\mathrm{L}^{2}(G) \otimes \mathrm{L}^{2}(G)\right)$, whose properties we will now describe. 
Let $\mathcal{H}$ be a Hilbert space. Recall that a multiplicative unitary is an element $\mathrm{W} \in$ $\mathcal{U}(\mathcal{H} \otimes \mathcal{H})$ that satisfies the pentagon equation

$$
\mathrm{W}_{23} \mathrm{~W}_{12}=\mathrm{W}_{12} \mathrm{~W}_{13} \mathrm{~W}_{23} \quad \text { in } \mathcal{U}(\mathcal{H} \otimes \mathcal{H} \otimes \mathcal{H})
$$

Define $C:=\left\{\left(\omega \otimes \mathrm{id}_{\mathcal{H}}\right)(\mathrm{W}) \mid \omega \in \mathbb{B}(\mathcal{H})_{*}\right\}^{\mathrm{CLS}}$. When $\mathrm{W}$ is manageable (see [47, Theorem 1.5]), $C$ is a separable nondegenerate $C^{*}$-subalgebra of $\mathbb{B}(\mathcal{H})$. Moreover, the formula $\Delta_{C}(c):=\mathrm{W}(c \otimes 1) \mathrm{W}^{*}$ for $c \in C$ defines an element of $\operatorname{Mor}(C, C \otimes C)$ such that $\left(C, \Delta_{C}\right)$ is a Hopf $C^{*}$-algebra (see $\left.[42,47]\right)$, which is said to be generated by W.

In particular, when we return to the Kustermans-Vaes setup, we denote the $C^{*}$-algebra $C$ associated to $\mathrm{W}^{G}$ (with $\left.\mathcal{H}=\mathrm{L}^{2}(G)\right)$ as $\mathrm{C}_{0}(G)$. Note that $\mathrm{C}_{0}(G) \subset \mathrm{L}^{\infty}(G)$ and the respective coproducts are compatible and will both be denoted by $\Delta_{G}$ (or $\Delta$ if the context is clear).

The dual of a multiplicative unitary $\mathrm{W} \in \mathcal{U}(\mathcal{H} \otimes \mathcal{H})$ is given by the formula $\widehat{\mathrm{W}}:=$ $\Sigma \mathrm{W}^{*} \Sigma \in \mathcal{U}(\mathcal{H} \otimes \mathcal{H})$. Moreover, $\widehat{\mathrm{W}}$ is manageable whenever $\mathrm{W}$ is. It turns out that if we start from a locally compact quantum group $G$ we can associate to it another locally compact quantum group which we denote $\hat{G}$ and call the dual locally compact quantum group of $G$, so that $\mathrm{W}^{\hat{G}}=\widehat{\mathrm{W}}$. We naturally have $\mathrm{C}_{0}(\hat{G}):=\left\{\left(\omega \otimes \mathrm{id}_{\mathcal{H}}\right)(\widehat{\mathrm{W}}) \mid \omega \in\right.$ $\left.\mathbb{B}(\mathcal{H})_{*}\right\}^{\mathrm{CLS}}$ and $\hat{\Delta}(\hat{c}):=\widehat{\mathrm{W}}(\hat{c} \otimes 1) \widehat{\mathrm{W}}^{*}$ for all $\hat{c} \in \mathrm{C}_{0}(\hat{G})$. Since the dual of $\widehat{\mathrm{W}}$ is equal to $\mathrm{W}$, we obtain a canonical isomorphism $\hat{\hat{G}} \approx G$.

Example 2.3. Let $G$ be a locally compact group and $\mu$ be its right Haar measure. Then the operator $\mathrm{W}^{G} \in \mathcal{U}\left(\mathrm{L}^{2}(G, \mu) \otimes \mathrm{L}^{2}(G, \mu)\right)$ defined by $\left(\mathrm{W}^{G} f\right)\left(g_{1}, g_{2}\right)=f\left(g_{1} g_{2}, g_{2}\right)$, $f \in \mathrm{L}^{2}(G, \mu), g_{1}, g_{2} \in G$, is a (manageable) multiplicative unitary. The resulting locally compact quantum group is given simply by the algebra $\mathrm{C}_{0}(G)$ with the comultiplication determined by the formula $\left(\Delta_{G} f\right)\left(g_{1}, g_{2}\right)=f\left(g_{1} g_{2}\right)$ for all $f \in \mathrm{C}_{0}(G)$, $g_{1}, g_{2} \in G$. Further we have $\mathrm{C}_{0}(\hat{G})=\mathrm{C}_{\mathrm{r}}^{*}(G)$, and the dual comultiplication is determined by the formula $\hat{\Delta}_{G}\left(\lambda_{g}\right)=\lambda_{g} \otimes \lambda_{g}$ for all $g \in G$.

Let then $G$ be a locally compact quantum group in the sense described above. We then write $1_{G}$ for the identity element of $\mathcal{M}\left(\mathrm{C}_{0}(G)\right)$. By virtue of [48, Theorem 1.3] and [28, Theorem 3.16 and Proposition 3.18], every compact quantum group can be also viewed as a locally compact quantum group; for a compact quantum group $G$ we naturally write $\mathrm{C}(G)$ for the (unital) $\mathrm{C}^{*}$-algebra $\mathrm{C}_{0}(G)$. Discrete quantum groups are duals of CQGs.

Definition 2.4. Let $\left(C, \Delta_{C}\right)$ be a Hopf $C^{*}$-algebra and $D$ be a $C^{*}$-algebra.

(1) An element $U \in \mathcal{U}(D \otimes C)$ is said to be a right corepresentation of $C$ in $D$ if and only if $\left(\operatorname{id}_{D} \otimes \Delta_{C}\right)(\mathrm{U})=\mathrm{U}_{12} \mathrm{U}_{13}$ in $\mathcal{U}(D \otimes C \otimes C)$.

(2) Similarly, a left corepresentation of $C$ in $D$ is an element $\mathrm{U} \in \mathcal{U}(C \otimes D)$ satisfying $\left(\Delta_{C} \otimes \mathrm{id}_{D}\right)(\mathrm{U})=\mathrm{U}_{23} \mathrm{U}_{13}$ in $\mathcal{U}(C \otimes C \otimes D)$.

(3) In particular, if $D=\mathbb{K}(\mathcal{L})$ for some Hilbert space $\mathcal{L}$, then $U$ is said to be (right or left) corepresentation of $C$ on $\mathcal{L}$.

An element $\mathrm{U} \in \mathcal{U}(D \otimes C)$ is a right corepresentation of $C$ in $D$ if and only if $\hat{\mathrm{U}}:=$ $\sigma\left(\mathrm{U}^{*}\right) \in \mathcal{U}(C \otimes D)$ is a left corepresentation of $C$ in $D$. From now on we reserve the word "corepresentation" for right corepresentations. Moreover if $G$ is a locally compact quantum group then we call right corepresentations of $\mathrm{C}_{0}(G)$ simply (unitary, strongly continuous) representations of $G$. 
Consider a locally compact quantum group $G$ and the manageable multiplicative unitary $\mathrm{W}^{G}$. Then $\mathrm{W}^{G} \in \mathcal{U}\left(\mathrm{C}_{0}(\hat{G}) \otimes \mathrm{C}_{0}(G)\right) \subset \mathcal{U}\left(\mathrm{L}^{2}(G) \otimes \mathrm{L}^{2}(G)\right)$. Thus we can also view $\mathrm{W}^{G}$ as a unitary element of the abstract $\mathrm{C}^{*}$-algebra $\mathcal{M}\left(\mathrm{C}_{0}(\hat{G}) \otimes \mathrm{C}_{0}(G)\right)$, called the reduced bicharacter of $G$. Indeed, it satisfies the following bicharacter conditions (see Definition 2.8):

$$
\begin{array}{ll}
\left(\mathrm{id}_{\mathrm{C}_{0}(\hat{G})} \otimes \Delta_{G}\right)\left(\mathrm{W}^{G}\right)=\mathrm{W}_{12}^{G} \mathrm{~W}_{13}^{G} & \text { in } \mathcal{U}\left(\mathrm{C}_{0}(\hat{G}) \otimes \mathrm{C}_{0}(G) \otimes \mathrm{C}_{0}(G)\right) ; \\
\left(\Delta_{\hat{G}} \otimes \operatorname{id}_{\mathrm{C}_{0}(G)}\right)\left(\mathrm{W}^{G}\right)=\mathrm{W}_{23}^{G} \mathrm{~W}_{13}^{G} & \text { in } \mathcal{U}\left(\mathrm{C}_{0}(\hat{G}) \otimes \mathrm{C}_{0}(\hat{G}) \otimes \mathrm{C}_{0}(G)\right) .
\end{array}
$$

Therefore, $\mathrm{W}^{G}$ is a representation of $G$ in $\mathrm{C}_{0}(\hat{G})$ and also a (left) representation of $\hat{G}$ in $\mathrm{C}_{0}(G)$.

Finally, note that in fact the Haar weights will not play any significant role in this paper and we will concentrate on the $\mathrm{C}^{*}$-algebraic setup.

2.2. Universal algebras of locally compact quantum groups. Suppose $G$ is a locally compact group. If $G$ is not amenable (for example, $G=\mathbb{F}_{2}$ ), the convolution algebra of compactly supported continuous functions on $G$ can have more than one $\mathrm{C}^{*}$ algebraic completion. By completing the convolution algebra of $G$ in the norm topology of $\mathcal{B}\left(L^{2}(G)\right)$, we get the reduced group $C^{*}-$ algebra $\mathrm{C}_{\mathrm{r}}^{*}(G)$. On the other hand, the quantity

$$
\|f\|_{\mathrm{u}}:=\sup _{\pi}\left\{\|\pi(f)\|: \pi \text { is a } *-\text { representation of } \mathrm{C}_{c}(G)\right\}
$$

defines a pre- $\mathrm{C}^{*}$-algebraic norm on the convolution algebra $\mathrm{C}_{c}(G)$. The completion of $\mathrm{C}_{c}(G)$ with respect to $\|\cdot\|_{\mathrm{u}}$ defines a $C^{*}$ algebra known as the full group $\mathrm{C}^{*}$ algebra of $G$ and denoted by $\mathrm{C}^{*}(G)$. There always exists a canonical surjective $\mathrm{C}^{*}$-homomorphism from $\mathrm{C}^{*}(G)$ to $\mathrm{C}_{\mathrm{r}}^{*}(G)$. When $G$ is not amenable, this homomorphism is not one-to-one. For more details, we refer to [36]. This explains the need to associate with any locally compact quantum group, apart from the 'reduced algebra of functions', discussed in the last subsection, also its 'universal' counterpart, which we describe in what follows.

Let $G$ be a locally compact quantum group. By [27] (see also [42, Proposition 22 and Theorem 25]), the algebra $\mathrm{C}_{0}(G)$ admits also a universal version, denoted $\mathrm{C}_{0}^{\mathrm{u}}(G)$, characterized by the fact that there exists a canonical 1-1 correspondence between representations of $\hat{G}$ and $C^{*}$-algebraic representations of $C_{0}^{u}(G)$, implemented by the 'semi-universal' version of the multiplicative unitary $\mathrm{W}^{G}$, denoted $\mathrm{W}^{G}$. We have $\mathrm{W}^{G} \in \mathcal{M}\left(\mathrm{C}_{0}(\hat{G}) \otimes \mathrm{C}_{0}^{\mathrm{u}}(G)\right)$, and $\mathrm{W}^{G}$ is in fact a representation of $\hat{G}$ in $\mathrm{C}_{0}^{\mathrm{u}}(G)$. The correspondence mentioned above is of the following form: given any (left) representation $\mathrm{U} \in \mathcal{U}\left(\mathrm{C}_{0}(\hat{G}) \otimes D\right)$ of $\hat{G}$ in a $\mathrm{C}^{*}$-algebra $D$ there is a unique $\varphi \in \operatorname{Mor}\left(\mathrm{C}_{0}^{\mathrm{u}}(G), D\right)$ with

$$
(\mathrm{id} \otimes \varphi)\left(\mathrm{W}^{G}\right)=\mathrm{U} .
$$

The algebra $\mathrm{C}_{0}^{\mathrm{u}}(G)$ is equipped with the comultiplication $\Delta^{\mathrm{u}} \in \operatorname{Mor}\left(\mathrm{C}_{0}^{\mathrm{u}}(G), \mathrm{C}_{0}^{\mathrm{u}}(G) \otimes\right.$ $\mathrm{C}_{0}^{\mathrm{u}}(G)$ ) (as well as a 'bi-universal multiplicative unitary', see below) making $\mathrm{C}_{0}^{\mathrm{u}}(G) \mathrm{a}$ Hopf $C^{*}$-algebra (see [42, Proposition 31]). By certain abuse of notation we will write $\mathrm{id}_{G}$ for both $\mathrm{id}_{\mathrm{C}_{0}(G)}$ and $\mathrm{id}_{\mathrm{C}_{0}^{\mathrm{u}}(G)}$, with the specific meaning clear from the context. For clarity, we will also sometimes write $\Delta_{G}^{\mathrm{u}}$ for $\Delta^{\mathrm{u}}$.

Further, note that the universality of $\mathrm{W}^{G}$ gives a unique $\Lambda_{G} \in \operatorname{Mor}\left(\mathrm{C}_{0}^{\mathrm{u}}(G), \mathrm{C}_{0}(G)\right)$, known as the reducing morphism, satisfying the equations

$$
\left(\mathrm{id} \otimes \Lambda_{G}\right)\left(\mathrm{W}^{G}\right)=\mathrm{W}^{G}, \quad\left(\Lambda_{G} \otimes \Lambda_{G}\right) \circ \Delta^{\mathrm{u}}=\Delta \circ \Lambda_{G} .
$$


Another application of the universal property yields the existence of the character $e: \mathrm{C}_{0}^{\mathrm{u}}(G) \rightarrow \mathbb{C}$, called the counit, associated to the trivial representation of $\hat{G}$ :

$$
(\mathrm{id} \otimes e)(\mathrm{W})=1_{\mathrm{C}_{0}(\hat{G})},
$$

with the following property:

$$
(e \otimes \mathrm{id}) \circ \Delta^{\mathrm{u}}=(\mathrm{id} \otimes e) \circ \Delta^{\mathrm{u}}=\mathrm{id}_{\mathrm{C}_{0}^{\mathrm{u}}(G)} .
$$

Naturally the construction described above can be applied also to the dual locally compact quantum group $\hat{G}$, yielding the (Hopf)- $C^{*}$-algebra $\mathrm{C}_{0}^{\mathrm{u}}(\hat{G})$, the unitary $\mathbb{W}^{G} \in$ $\mathcal{M}\left(\mathrm{C}_{0}^{\mathrm{u}}(\hat{G}) \otimes \mathrm{C}_{0}(G)\right)$, reducing morphism $\Lambda_{\hat{G}}$, etc. In fact Kustermans showed the existence of the 'fully universal' multiplicative unitary $\mathbb{W}^{G} \in \mathcal{M}\left(\mathrm{C}_{0}^{\mathrm{u}}(\hat{G}) \otimes \mathrm{C}_{0}^{\mathrm{u}}(G)\right)$ such that

$$
\mathrm{W}^{G}=\left(\Lambda_{\hat{G}} \otimes \Lambda_{G}\right)\left(\mathrm{W}^{G}\right) .
$$

We call $G$ coamenable if the reducing morphism $\Lambda_{G}$ is an isomorphism. This is the case for $G$ classical or discrete. On the other hand the dual of a classical locally compact group $G$ is coamenable if and only if $G$ is amenable. Indeed, we have $\mathrm{C}_{0}^{\mathrm{u}}(\hat{G})=\mathrm{C}^{*}(G)$, with the comultiplication $\Delta_{\hat{G}}^{\mathrm{u}}\left(u_{g}\right)=u_{g} \otimes u_{g}$ for all $g \in G$, where $u_{g}$ denotes the image of $g$ under the universal representation. By analogy with the notation introduced before, for a compact quantum group $G$ we will write simply $\mathrm{C}^{u}(G)$ for $\mathrm{C}_{0}^{\mathrm{u}}(G)$. Each of the algebras $\mathrm{C}(G)$ and $\mathrm{C}^{u}(G)$ contain then a canonical dense Hopf *-subalgebra, $\operatorname{Pol}(G)$, and in fact $\operatorname{Pol}(G)$ may admit also other, so-called exotic completions to Hopf $\mathrm{C}^{*}$-algebras (see [30]).

2.3. Coactions, bicharacters and quantum group morphisms. In this short subsection we discuss quantum group actions and the notion of morphisms between locally compact quantum groups.

Definition 2.5. Let $\left(C, \Delta_{C}\right)$ be a Hopf $C^{*}$-algebra. A (right) coaction of $C$ on a $C^{*}$-algebra $A$ is an element $\gamma \in \operatorname{Mor}(A, A \otimes C)$ such that

(1) $\gamma$ is a comodule structure (in other words, it satisfies the action equation), that is,

$$
\left(\mathrm{id}_{A} \otimes \Delta_{C}\right) \circ \gamma=\left(\gamma \otimes \mathrm{id}_{C}\right) \circ \gamma
$$

(2) $\gamma$ satisfies the Podleś condition:

$$
\gamma(A)\left(1_{A} \otimes C\right)=A \otimes C .
$$

In the case where $\left(C, \Delta_{C}\right)=\left(\mathrm{C}_{0}(G), \Delta_{G}\right)$ for a locally compact quantum group $G$, we call $\gamma$ simply the (reduced) action of $G$ on $A$, and the pair $(A, \gamma)$ is called a $G$-C $C^{*}$-algebra. Sometimes one needs also to consider universal actions of $G$ (i.e. the coactions of the Hopf $\mathrm{C}^{*}$-algebra $\left.\mathrm{C}_{0}^{\mathrm{u}}(G)\right)$.

Let us record a consequence of the Podleś condition for *-homomorphisms.

Lemma 2.6. Let $A, B, C$ be $C^{*}$-algebras with $A \subseteq \mathcal{M}(D)$ and let $\gamma: A \rightarrow \mathcal{M}(D \otimes C)$ be $a^{*}$-homomorphism such that $\gamma(A)\left(1_{D} \otimes C\right)=A \otimes C$. Then $\gamma \in \operatorname{Mor}(A, A \otimes C)$. 
Proof. The Podleś condition implies that $\gamma(A)(A \otimes C)=\gamma(A)\left(1_{D} \otimes C\right)\left(A \otimes 1_{C}\right)=$ $(A \otimes C)\left(A \otimes 1_{C}\right)=A \otimes C$. Applying the adjoint to the last equality we also get $(A \otimes C) \gamma(A)=A \otimes C$. Thus, $\gamma \in \operatorname{Mor}(A, A \otimes C)$.

A covariant representation of the coaction $(A, \gamma)$ on a Hilbert space $\mathcal{H}$ is a pair $(\mathrm{U}, \varphi)$ consisting of a corepresentation $\mathrm{U} \in \mathcal{U}(\mathbb{K}(\mathcal{H}) \otimes C)$ of $C$ on $\mathcal{H}$ and a representation $\varphi \in \operatorname{Mor}(A, \mathbb{K}(\mathcal{H}))$ that satisfy the covariance condition

$$
\left(\varphi \otimes \operatorname{id}_{C}\right)(\gamma(a))=\mathrm{U}\left(\varphi(a) \otimes 1_{C}\right) \mathrm{U}^{*}, \quad a \in A .
$$

A covariant representation is called faithful if $\varphi$ is faithful. Faithful covariant representations always exist whenever $\gamma$ is injective (see [35, Example 4.5]).

In this article we are mainly going to work with compact quantum groups $G$ and unital $G$-C $C^{*}$-algebras $(A, \gamma)$ with a faithful state $\tau$ on $A$ such that $\gamma$ preserves $\tau$, i.e.

$$
\left(\tau \otimes \operatorname{id}_{G}\right)(\gamma(a))=\tau(a) 1_{G} \quad \text { for all } a \in A,
$$

Let us note that such actions are always injective.

Lemma 2.7. Let $(A, \gamma)$ be a unital $G-C^{*}$-algebra with a faithful state $\tau$ such that $\gamma$ preserves $\tau$. Then $\gamma$ is injective.

We will now define bicharacters associated to two given Hopf $\mathrm{C}^{*}$-algebras. This notion plays a fundamental role in describing morphisms between locally compact quantum groups.

Definition 2.8. Let $\left(C, \Delta_{C}\right)$ and $\left(D, \Delta_{D}\right)$ be Hopf $C^{*}$-algebras. An element $\mathrm{V} \in \mathcal{U}(C \otimes$ $D)$ is said to be a bicharacter if it satisfies the following properties

$$
\begin{array}{ll}
\left(\Delta_{C} \otimes \operatorname{id}_{D}\right)(\mathrm{V})=\mathrm{V}_{23} \mathrm{~V}_{13} & \text { in } \mathcal{U}(C \otimes C \otimes D), \\
\left(\operatorname{id}_{C} \otimes \Delta_{D}\right)(\mathrm{V})=\mathrm{V}_{12} \mathrm{~V}_{13} & \text { in } \mathcal{U}(C \otimes D \otimes D) .
\end{array}
$$

The notion of bicharacter for quantum groups is a generalization of that of bicharacters for groups. Indeed, let $G$ and be $H$ be locally compact abelian groups. An element $\mathrm{V} \in$ $\mathcal{U}\left(\mathrm{C}_{0}(\hat{G}) \otimes \mathrm{C}_{0}(\hat{H})\right)$ is simply a continuous map $\mathrm{V}: \hat{G} \times \hat{H} \rightarrow \mathbb{T}$ and the conditions (2.11) and (2.12) say that this map is a bicharacter in the classical sense.

Let $G$ and $H$ be locally compact quantum groups.

Example 2.9. A Hopf*-homomorphism $f \in \operatorname{Mor}\left(\mathrm{C}_{0}(G), \mathrm{C}_{0}(H)\right)$ induces a bicharacter $\mathrm{V}_{f} \in \mathcal{U}\left(\mathrm{C}_{0}(\hat{G}) \otimes \mathrm{C}_{0}(H)\right)$ defined by $\mathrm{V}_{f}:=\left(\operatorname{id}_{\hat{G}} \otimes f\right)\left(\mathrm{W}^{G}\right)$. The 'semi-universal' multiplicative unitaries $\mathrm{W} \in \mathcal{U}\left(\mathrm{C}_{0}^{\mathrm{u}}(\hat{G}) \otimes \mathrm{C}_{0}(G)\right)$ and $\mathrm{W} \in \mathcal{U}\left(\mathrm{C}_{0}(\hat{G}) \otimes \mathrm{C}_{0}^{\mathrm{u}}(G)\right)$ are both bicharacters.

Bicharacters in $\mathcal{U}\left(\mathrm{C}_{0}(\hat{G}) \otimes \mathrm{C}_{0}(H)\right)$ are interpreted as quantum group morphisms from $G$ to $H$. The article [34] contains a detailed study of such morphisms and provides several equivalent pictures. Since the notion of bicharacters is going to be crucially used in the article, let us recall some of the related definitions and results from that paper. For simplicity we will describe below quantum group morphisms from $G$ to $\hat{H}$.

A right quantum group homomorphism from $G$ to $\hat{H}$ is an element $\Delta_{R} \in \operatorname{Mor}\left(\mathrm{C}_{0}(G)\right.$, $\left.\mathrm{C}_{0}(G) \otimes \mathrm{C}_{0}(\hat{H})\right)$ with the following properties:

$\left(\Delta_{G} \otimes \mathrm{id}_{\hat{H}}\right) \circ \Delta_{R}=\left(\mathrm{id}_{G} \otimes \Delta_{R}\right) \circ \Delta_{G} \quad$ and $\quad\left(\mathrm{id}_{G} \otimes \Delta_{\hat{H}}\right) \circ \Delta_{R}=\left(\Delta_{R} \otimes \mathrm{id}_{\hat{H}}\right) \circ \Delta_{R}$. 
Theorem 2.10 [34]. Let $G$ and $H$ be locally compact quantum groups. There are natural bijections between the following sets:

(1) bicharacters $\mathrm{V} \in \mathcal{U}\left(\mathrm{C}_{0}(\hat{G}) \otimes \mathrm{C}_{0}(\hat{H})\right)$;

(2) bicharacters $\widehat{\mathrm{V}} \in \mathcal{U}\left(\mathrm{C}_{0}(\hat{H}) \otimes \mathrm{C}_{0}(\hat{G})\right)$;

(3) right quantum group homomorphisms $\Delta_{R} \in \operatorname{Mor}\left(\mathrm{C}_{0}(G), \mathrm{C}_{0}(G) \otimes \mathrm{C}_{0}(\hat{H})\right)$;

(4) Hopf*-homomorphisms $f \in \operatorname{Mor}\left(\mathrm{C}_{0}^{\mathrm{u}}(G), \mathrm{C}_{0}^{\mathrm{u}}(\hat{H})\right)$.

The first bijection maps a bicharacter $\mathrm{V}$ to its dual $\widehat{\mathrm{V}} \in \mathcal{U}\left(\mathrm{C}_{0}(\hat{H}) \otimes \mathrm{C}_{0}(\hat{G})\right)$ defined by

$$
\widehat{\mathrm{V}}:=\sigma\left(\mathrm{V}^{*}\right) .
$$

A bicharacter $\mathrm{V}$ and a right quantum group homomorphism $\Delta_{R}$ determine each other uniquely via

$$
\left(\operatorname{id}_{\hat{G}} \otimes \Delta_{R}\right)\left(\mathrm{W}^{G}\right)=\mathrm{W}_{12}^{G} \mathrm{~V}_{13} .
$$

A bicharacter $\mathrm{V} \in \mathcal{U}\left(\mathrm{C}_{0}(\hat{G}) \otimes \mathrm{C}_{0}(\hat{H})\right)$ and aHopf*-homomorphism $f \in \operatorname{Mor}\left(\mathrm{C}_{0}^{\mathrm{u}}(G)\right.$, $\left.\mathrm{C}_{0}^{\mathrm{u}}(\hat{H})\right)$ determine each other uniquely by

$$
\left(\operatorname{id}_{G} \otimes \Lambda_{\hat{H}^{\circ}} \circ\right)\left(\mathrm{W}^{G}\right)=\mathrm{V} .
$$

The dual bicharacter $\widehat{\mathrm{V}} \in \mathcal{U}\left(\mathrm{C}_{0}(\hat{H}) \otimes \mathrm{C}_{0}(\hat{G})\right)$ should be thought of as the dual quantum group morphism from $H$ to $\hat{G}$. It corresponds to the dual right quantum group homomorphism $\hat{\Delta}_{R} \in \operatorname{Mor}\left(\mathrm{C}_{0}(\hat{H}), \mathrm{C}_{0}(\hat{H}) \otimes \mathrm{C}_{0}(G)\right)$. Thus $\Delta_{R}$ and $\hat{\Delta}_{R}$ are in bijection as $\mathrm{V}$ and $\mathrm{V}$ are. Finally, the dual bicharacter describes a unique Hopf*-homomorphism $\hat{f} \in \operatorname{Mor}\left(\mathrm{C}_{0}^{\mathrm{u}}(H), \mathrm{C}_{0}^{\mathrm{u}}(\hat{G})\right)$. Thus $f$ and $\hat{f}$ determine each other uniquely by

$$
\left(\operatorname{id}_{G} \otimes \Lambda_{\hat{H}} \circ f\right)\left(\mathrm{W}^{G}\right)=\mathrm{V}=\sigma\left(\left(\operatorname{id}_{H} \otimes \Lambda_{\hat{G}} \circ \hat{f}\right)\left(\mathrm{W}^{H}\right)^{*}\right) .
$$

Let $\gamma^{\mathrm{u}}$ be a coaction of $\mathrm{C}_{0}^{\mathrm{u}}(G)$ on a $\mathrm{C}^{*}$-algebra $A$. It is said to be normal if the associated reduced action of $G$ on $A, \gamma:=\left(\operatorname{id}_{A} \otimes \Lambda_{G}\right) \circ \gamma^{\mathrm{u}}$, is injective. By virtue of [40, Theorem A.2] or [19], an injective coaction of $\mathrm{C}_{0}(G)$ on a $\mathrm{C}^{*}$-algebra $A$ lifts uniquely to a normal coaction of $\mathrm{C}_{0}^{\mathrm{u}}(G)$ on $A$.

Yet another equivalent description of quantum group homomorphisms [34, Section 6] shows that for an injective coaction $\gamma \in \operatorname{Mor}\left(A, A \otimes \mathrm{C}_{0}(G)\right)$ and a right quantum group homomorphism $\Delta_{R} \in \operatorname{Mor}\left(\mathrm{C}_{0}(G), \mathrm{C}_{0}(G) \otimes \mathrm{C}_{0}(\hat{H})\right)$ there is a unique injective coaction $\delta \in \operatorname{Mor}\left(A, A \otimes \mathrm{C}_{0}(\hat{H})\right)$ such that the following diagram commutes:

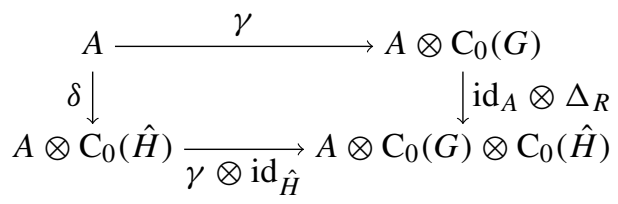

Let $\gamma^{\mathrm{u}}$ denote the associated normal coaction of $\mathrm{C}_{0}^{\mathrm{u}}(G)$ on $A$ and let $f \in \operatorname{Mor}\left(\mathrm{C}_{0}^{\mathrm{u}}(G), \mathrm{C}_{0}^{\mathrm{u}}(\hat{H})\right)$ be the Hopf*-homomorphism corresponding to $\Delta_{R}$. Then we say that $\delta$ is induced from $\gamma$ by $\Delta_{R}$ or $f$. The following lemma describes $\delta$ explicitly.

Lemma 2.11. The coaction $\delta$ in (2.17) is given by $\delta:=\left(\operatorname{id}_{A} \otimes \Lambda_{\hat{H}} \circ f\right) \circ \gamma^{\mathrm{u}}$. 
Proof. By the uniqueness of $\delta$, it is enough to show that $\delta$ satisfies the equation

$$
\left(\mathrm{id}_{A} \otimes \Delta_{R}\right) \circ \gamma=\left(\gamma \otimes \mathrm{id}_{\hat{H}}\right) \circ \delta .
$$

Let us recall [40, Equation 2.23]: $\Delta_{R} \circ \Lambda_{G}=\left(\Lambda_{G} \otimes \Lambda_{\hat{H}} \circ f\right) \circ \Delta_{G}^{\mathrm{u}}$. Using this equation and properties of $\gamma$ and $\gamma^{\mathrm{u}}$ we obtain

$$
\begin{aligned}
\left(\mathrm{id}_{A} \otimes \Delta_{R}\right) \circ \gamma=\left(\mathrm{id}_{A} \otimes \Delta_{R} \circ \Lambda_{G}\right) \circ \gamma^{\mathrm{u}} & =\left(\mathrm{id}_{A} \otimes\left(\Lambda_{G} \otimes \Lambda_{\hat{H}} \circ f\right) \circ \Delta_{G}^{\mathrm{u}}\right) \circ \gamma^{\mathrm{u}} \\
& =\left(\left(\operatorname{id}_{A} \otimes \Lambda_{G}\right) \circ \gamma^{\mathrm{u}} \otimes \Lambda_{\hat{H}} \circ f\right) \circ \gamma^{\mathrm{u}} \\
& =\left(\gamma \otimes \mathrm{id}_{\hat{H}}\right) \circ \delta .
\end{aligned}
$$

2.4. Twisted tensor product $\mathrm{C}^{*}$-algebras and the action of the Drinfeld double. In this subsection we recall the notion of quantum group twisted tensor product of $\mathrm{C}^{*}$-algebras as developed in [35] and the action of the generalized Drinfeld double on the twisted tensor product constructed in [39].

We start with the following data: let $G$ and $H$ be locally compact quantum groups, let $\left(A, \gamma_{A}\right),\left(B, \gamma_{B}\right)$, be a $G-C^{*}$-algebra, respectively a $H$-C $C^{*}$-algebra, such that $\gamma_{A}$ and $\gamma_{B}$ are injective maps and let $\mathrm{V} \in \mathcal{U}\left(\mathrm{C}_{0}(\hat{G}) \otimes \mathrm{C}_{0}(\hat{H})\right)$ be a bicharacter (viewed as a morphism from $G$ to $\hat{H}$ ).

By [35, Lemma 3.8], there exists a V-Heisenberg pair, i.e. a Hilbert space $\mathcal{H}$ and a pair of representations $\alpha \in \operatorname{Mor}\left(\mathrm{C}_{0}(G), \mathbb{K}(\mathcal{H})\right)$ and $\beta \in \operatorname{Mor}\left(\left(\mathrm{C}_{0}(H), \mathbb{K}(\mathcal{H})\right)\right.$ that satisfy the following condition:

$$
\mathrm{W}_{1 \alpha}^{G} \mathrm{~W}_{2 \beta}^{H}=\mathrm{W}_{2 \beta}^{H} \mathrm{~W}_{1 \alpha}^{G} \mathrm{~V}_{12} \quad \text { in } \mathcal{U}\left(\mathrm{C}_{0}(\hat{G}) \otimes \mathrm{C}_{0}(\hat{H}) \otimes \mathbb{K}(\mathcal{H})\right) .
$$

Here, $\mathrm{W}_{1 \alpha}^{G}:=\left(\left(\operatorname{id}_{\hat{G}} \otimes \alpha\right)\left(\mathrm{W}^{G}\right)\right)_{13}$ and $\mathrm{W}_{2 \beta}^{H}:=\left(\left(\operatorname{id}_{\hat{H}} \otimes \beta\right)\left(\mathrm{W}^{H}\right)\right)_{23}$. We recall [35, Example 3.2] to motivate the notion of V-Heisenberg pairs. Indeed, let $G=H=\mathbb{R}$ and consider a standard bicharacter $\mathrm{V} \in \mathcal{U}\left(\mathrm{C}_{0}(\mathbb{R}) \otimes \mathrm{C}_{0}(\mathbb{R})\right)$, i.e. the map $(s, t) \rightarrow \exp ($ ist $)$. Then the maps $\alpha$ and $\beta$ satisfying the condition (2.18) can be equivalently described as a pair of continuous one-parameter unitary groups $\left(U_{1}(s), U_{2}(t)\right)_{s, t \in \mathbb{R}}$ satisfying the canonical commutation relation in the Weyl form: $U_{1}(s) U_{2}(t)=\exp (i s t) U_{2}(t) U_{1}(s)$ for all $s, t \in \mathbb{R}$.

Define $j_{A} \in \operatorname{Mor}(A, A \otimes B \otimes \mathbb{K}(\mathcal{H}))$ and $j_{B} \in \operatorname{Mor}(B, A \otimes B \otimes \mathbb{K}(\mathcal{H}))$ by

$$
j_{A}(a):=\left(\left(\operatorname{id}_{A} \otimes \alpha\right)\left(\gamma_{A}(a)\right)\right)_{13}, \quad j_{B}(b):=\left(\left(\operatorname{id}_{B} \otimes \alpha\right)\left(\gamma_{B}(b)\right)\right)_{23}
$$

for all $a \in A, b \in B$.

Then we have the following theorem.

Theorem 2.12 ([35, Theorem 4.6]). The space $A \bigotimes_{\mathrm{V}} B:=j_{A}(A) j_{B}(B)$ is a $\mathrm{C}^{*}$-algebra, which does not depend (up to an isomorphism) on the choice of the V-Heisenberg pair $(\alpha, \beta)$.

Let $\mathrm{V}=1 \in \mathcal{U}\left(\mathrm{C}_{0}(\hat{G}) \otimes \mathrm{C}_{0}(\hat{H})\right)$ be the trivial bicharacter. The associated twisted tensor product of $A$ and $B$ is isomorphic to the minimal tensor product $A \otimes B$. Further, if $\Gamma$ is a discrete group acting on a unital $C^{*}$-algebra $A$ via an action $\beta$, then the reduced crossed product $A \rtimes_{\beta, \mathrm{r}} \Gamma$ is another particular example of the above construction. We formalize this observation in the theorem below. 
Proposition 2.13 ([35, Theorem 6.3]). Let $\Gamma$ be a discrete group, and assume that $\gamma_{A}$ : = $\beta \in \operatorname{Mor}\left(A, A \otimes \mathrm{C}_{0}(\Gamma)\right)$ is a coaction of $\mathrm{C}_{0}(\Gamma)$ on a $\mathrm{C}^{*}$-algebra $A$, that $\left(B, \gamma_{B}\right)=$ $\left(\mathrm{C}_{\mathrm{r}}^{*}(\Gamma), \hat{\Delta}_{\Gamma}\right)\left(\right.$ i.e. $\gamma_{B}$ is a canonical action of $\hat{\Gamma}$ on itself $)$ and that $\mathrm{V}=\mathrm{W}^{\Gamma} \in \mathcal{U}\left(\mathrm{C}_{\mathrm{r}}^{*}(\Gamma) \otimes\right.$ $\left.\mathrm{C}_{0}(\Gamma)\right)$ is the reduced bicharacter for $\Gamma$. Then there exists an isomorphism $\Psi: A \bigotimes_{\mathrm{V}} B \rightarrow$ $A \rtimes_{\beta, \mathrm{r}} \Gamma$ such that for all $a \in A, b \in B$,

$$
\Psi\left(j_{A}(a)\right)=\beta(a), \quad \Psi\left(j_{B}(b)\right)=1 \otimes b .
$$

Here we have used the canonical faithful representations of $\mathrm{C}_{0}(\Gamma)$ and $\mathrm{C}_{\mathrm{r}}^{*}(\Gamma)$ in $\mathbb{B}\left(\ell^{2}(\Gamma)\right)$ in the second legs.

The generalized Drinfeld double $\mathfrak{D}_{\mathrm{V}}$ of $G$ and $H$ with respect to the bicharacter $\mathrm{V}$ is a locally compact quantum group described (see [39, Theorem 5.1(ii)]) as follows:

$$
\begin{aligned}
\mathrm{C}_{0}\left(\mathfrak{D}_{\mathrm{V}}\right) & =\rho\left(\mathrm{C}_{0}(G)\right) \theta\left(\mathrm{C}_{0}(H)\right), \\
\Delta_{\mathfrak{D}_{\mathrm{V}}}(\rho(x) \theta(y)) & =(\rho \otimes \rho)\left(\Delta_{G}(x)\right)(\theta \otimes \theta)\left(\Delta_{H}(y)\right)
\end{aligned}
$$

for all $x \in \mathrm{C}_{0}(G)$ and $y \in \mathrm{C}_{0}(H)$. Here $\rho$ and $\theta$ form a pair of faithful representations of $\mathrm{C}_{0}(G)$ and $\mathrm{C}_{0}(H)$ on a Hilbert space $\mathcal{H}_{\mathcal{D}}$ that satisfies V-Drinfeld commutation relation:

$$
\mathrm{V}_{12} \mathrm{~W}_{1 \rho}^{G} \mathrm{~W}_{2 \theta}^{H}=\mathrm{W}_{2 \theta}^{H} \mathrm{~W}_{1 \rho}^{G} \mathrm{~V}_{12} \quad \text { in } \mathcal{U}\left(\mathrm{C}_{0}(\hat{G}) \otimes \mathrm{C}_{0}(\hat{H}) \otimes \mathbb{K}\left(\mathcal{H}_{\mathcal{D}}\right)\right)
$$

where $\mathrm{W}_{1 \rho}^{G}:=\left(\left(\operatorname{id}_{\hat{G}} \otimes \rho\right)\left(\mathrm{W}^{G}\right)\right)_{13}$ and $\mathrm{W}_{2 \theta}^{H}:=\left(\left(\operatorname{id}_{H} \otimes \theta\right)\left(\mathrm{W}^{H}\right)\right)_{23}$.

In fact, $\rho \in \operatorname{Mor}\left(\mathrm{C}_{0}(G), \mathrm{C}_{0}\left(\mathfrak{D}_{\mathrm{V}}\right)\right)$ and $\theta \in \operatorname{Mor}\left(\mathrm{C}_{0}(H), \mathrm{C}_{0}\left(\mathfrak{D}_{\mathrm{V}}\right)\right)$ are Hopf*-homomorphisms.

Now, [39, Lemma 6.3] shows that there is a canonical coaction $\gamma_{A} \bowtie \mathrm{V} \quad \gamma_{B} \in$ $\operatorname{Mor}\left(A \nabla_{\mathrm{V}} B, A \otimes_{\mathrm{V}} B \otimes \mathrm{C}_{0}\left(\mathfrak{D}_{\mathrm{V}}\right)\right)$ of $\mathrm{C}_{0}\left(\mathfrak{D}_{\mathrm{V}}\right)$ on $A \otimes_{\mathrm{V}} B$ defined by

$$
\gamma_{A} \bowtie \mathrm{V} \gamma_{B}\left(j_{A}(a)\right)=\left(j_{A} \otimes \rho\right)\left(\gamma_{A}(a)\right), \quad \gamma_{A} \bowtie \gamma_{B}\left(j_{B}(b)\right)=\left(j_{B} \otimes \theta\right)\left(\gamma_{B}(b)\right)
$$

for all $a \in A, b \in B$.

Lemma 2.14. Suppose $\gamma_{A}$ and $\gamma_{B}$ are injective maps. Then the map $\gamma_{A} \bowtie \mathrm{V} \gamma_{B}$ defined by (2.24) is also injective.

Proof. Without loss of generality we may assume that $\left(\mathrm{U}^{A}, \varphi_{A}\right)$ and $\left(\mathrm{U}^{B}, \varphi_{B}\right)$ are faithful covariant representations of $\left(A, \gamma_{A}\right)$ and $\left(B, \gamma_{B}\right)$ on the Hilbert spaces $\mathcal{H}_{A}$ and $\mathcal{H}_{B}$, respectively.

There is a faithful representation $\Pi: A \otimes_{\mathrm{V}} B \rightarrow \mathbb{B}\left(\mathcal{H}_{A} \otimes \mathcal{H}_{B}\right)$ such that $\Pi\left(j_{A}(a)\right)=$ $\varphi_{A}(a) \otimes 1_{\mathcal{H}_{B}}$ and $\Pi\left(j_{B}(b)\right)=Z\left(1_{\mathcal{H}_{A}} \otimes \varphi_{B}(b)\right) Z^{*}$, where $Z \in \mathcal{U}\left(\mathcal{H}_{A} \otimes \mathcal{H}_{B}\right)$ is the unique unitary that satisfies

$$
\mathrm{U}_{1 \alpha}^{A} \mathrm{U}_{2 \beta}^{B} Z_{12}=\mathrm{U}_{2 \beta}^{B} \mathrm{U}_{1 \alpha}^{A} \quad \text { in } \mathcal{U}\left(\mathcal{H}_{A} \otimes \mathcal{H}_{B} \otimes \mathcal{H}\right)
$$

for any V-Heisenberg pair $(\alpha, \beta)$ on $\mathcal{H}$ (see [35, Theorem 4.1 and 4.2]). Define a pair of representations $\tilde{\alpha}:=(\alpha \otimes \rho) \circ \Delta_{G}$ and $\tilde{\beta}(y):=(\beta \otimes \theta) \circ \Delta_{H}$ of $\mathrm{C}_{0}(G)$ and $\mathrm{C}_{0}(H)$ on $\mathcal{H} \otimes \mathcal{H}_{\mathcal{D}}$. By Definition 2.4.(1) for $\mathrm{U}^{A}$ and $\mathrm{U}^{B}$ and (2.25) we have

$$
\mathrm{U}_{1 \tilde{\alpha}}^{A} \mathrm{U}_{2 \tilde{\beta}}^{B} Z_{12}=\mathrm{U}_{1 \alpha}^{A} \mathrm{U}_{1 \rho}^{A} \mathrm{U}_{2 \beta}^{B} \mathrm{U}_{2 \theta}^{B} Z_{12}=\mathrm{U}_{1 \alpha}^{A} \mathrm{U}_{2 \beta}^{B} \mathrm{U}_{1 \rho}^{A} \mathrm{U}_{2 \theta}^{B} Z_{12}=\mathrm{U}_{2 \beta}^{B} \mathrm{U}_{1 \alpha}^{A} Z_{12}^{*} \mathrm{U}_{1 \rho}^{A} \mathrm{U}_{2 \theta}^{B} Z_{12}
$$


in $\mathcal{U}\left(\mathcal{H}_{A} \otimes \mathcal{H}_{B} \otimes \mathcal{H} \otimes \mathcal{H}_{\mathcal{D}}\right)$. Here $\alpha$ and $\beta$ are acting on the third leg and $\rho$ and $\theta$ are acting on the fourth leg. Similarly, we have

$$
\mathrm{U}_{2 \tilde{\beta}}^{B} \mathrm{U}_{1 \tilde{\alpha}}^{A}=\mathrm{U}_{2 \beta}^{B} \mathrm{U}_{2 \theta}^{B} \mathrm{U}_{1 \alpha}^{A} \mathrm{U}_{1 \rho}^{A}=\mathrm{U}_{2 \beta}^{B} \mathrm{U}_{1 \alpha}^{A} \mathrm{U}_{2 \theta}^{B} \mathrm{U}_{1 \rho}^{A}
$$

Now $(\tilde{\alpha}, \tilde{\beta})$ is a V-Heisenberg pair on $\mathcal{H} \otimes \mathcal{H}_{\mathcal{D}}$ (see the proof of [39, Lemma 6.3]). Therefore, we have the same commutation relation (2.25) if we replace $\alpha$ by $\tilde{\alpha}$ and $\beta$ by $\tilde{\beta}$. This gives the equality

$$
\mathrm{U}_{1 \rho}^{A} \mathrm{U}_{2 \theta}^{B} Z_{12}=Z_{12} \mathrm{U}_{2 \theta}^{B} \mathrm{U}_{1 \rho}^{A} \quad \text { in } \mathcal{U}\left(\mathcal{H}_{A} \otimes \mathcal{H}_{B} \otimes \mathcal{H}_{\mathcal{D}}\right)
$$

Using (2.26) and (2.24) we compute (for $a \in A, b \in B$ )

$$
\begin{aligned}
& \mathrm{U}_{1 \rho}^{A} \mathrm{U}_{2 \theta}^{B}\left(\Pi\left(j_{A}(a) j_{B}(b)\right) \otimes 1_{\mathfrak{D}_{\mathrm{V}}}\right)\left(\mathrm{U}_{2 \theta}^{B}\right)^{*}\left(\mathrm{U}_{1 \rho}^{A}\right)^{*} \\
& \quad=\mathrm{U}_{1 \rho}^{A} \mathrm{U}_{2 \theta}^{B}\left(\left(\varphi_{A}(a) \otimes 1_{\mathcal{H}_{B}} \otimes 1_{\mathfrak{D}_{\mathrm{V}}}\right) Z_{12}\left(1_{\mathcal{H}_{A}} \otimes \varphi_{B}(b) \otimes 1_{\mathfrak{D}_{\mathrm{V}}}\right)\right) Z_{12}^{*}\left(\mathrm{U}_{2 \theta}^{B}\right)^{*}\left(\mathrm{U}_{1 \rho}^{A}\right)^{*} \\
& \quad=\mathrm{U}_{1 \rho}^{A}\left(\varphi_{A}(a) \otimes 1_{\mathcal{H}_{B}} \otimes 1_{\mathfrak{D}_{\mathrm{V}}}\right) \mathrm{U}_{2 \theta}^{B} Z_{12}\left(1_{\mathcal{H}_{A}} \otimes \varphi_{B}(b) \otimes 1_{\mathfrak{D}_{\mathrm{V}}}\right)\left(\mathrm{U}_{1 \rho}^{A}\right)^{*}\left(\mathrm{U}_{2 \theta}^{B}\right)^{*} Z_{12}^{*} \\
& \quad=\mathrm{U}_{1 \rho}^{A}\left(\varphi_{A}(a) \otimes 1_{\mathcal{H}_{B}} \otimes 1_{\mathfrak{D}_{\mathrm{V}}}\right) \mathrm{U}_{2 \theta}^{B} Z_{12}\left(\mathrm{U}_{1 \rho}^{A}\right)^{*}\left(1_{\mathcal{H}_{A}} \otimes \varphi_{B}(b) \otimes 1_{\mathfrak{D}_{\mathrm{V}}}\right)\left(\mathrm{U}_{2 \theta}^{B}\right)^{*} Z_{12}^{*} \\
& =\mathrm{U}_{1 \rho}^{A}\left(\varphi_{A}(a) \otimes 1_{\mathcal{H}_{B}} \otimes 1_{\mathfrak{D}_{\mathrm{V}}}\right)\left(\mathrm{U}_{1 \rho}^{A}\right)^{*} Z_{12} \mathrm{U}_{2 \theta}^{B}\left(1_{\mathcal{H}_{A}} \otimes \varphi_{B}(b) \otimes 1_{\mathfrak{D}_{\mathrm{V}}}\right)\left(\mathrm{U}_{2 \theta}^{B}\right)^{*} Z_{12}^{*} \\
& =\left(\left(\varphi_{1} \otimes \rho\right)\left(\gamma_{A}(a)\right)\right)_{13} Z_{12}\left(\left(\varphi_{2} \otimes \theta\right)\left(\gamma_{B}(b)\right)\right)_{23} Z_{12}^{*} \\
& =\left(\Pi \otimes \mathrm{id}_{\mathfrak{D}_{\mathrm{V}}}\right)\left(\left(j_{A} \otimes \rho\right)\left(\gamma_{A}(a)\right)\left(j_{B} \otimes \theta\right)\left(\gamma_{B}(b)\right)\right) \\
& =\left(\Pi \otimes \mathrm{id}_{\mathfrak{D}_{\mathrm{V}}}\right)\left(\gamma_{A} \bowtie \gamma_{B}\left(j_{A}(a) j_{B}(b)\right)\right) .
\end{aligned}
$$

Since $\Pi$ is injective we conclude that $\gamma_{A} \bowtie \gamma_{B}$ is also injective.

Finally, note the special (trivial) case of the above construction. The generalized Drinfeld double of locally compact groups $G$ and $H$ associated to the trivial bicharacter $\mathrm{V}=1 \in \mathcal{U}\left(\mathrm{C}_{0}(\hat{G}) \otimes \mathrm{C}_{0}(\hat{H})\right)$ is just the cartesian product of the initial groups: $\mathrm{C}_{0}\left(\mathfrak{D}_{\mathrm{V}}\right)=$ $\mathrm{C}_{0}(G \times H)$. Moreover, $\mathrm{C}_{0}(G \times H)$ canonically coacts (component-wise) on $A \otimes B$ which is a particular case of (2.24). Furthermore, for any locally compact quantum group $G$ the generalized Drinfeld double of $G$ and $\hat{G}$ with respect to the reduced bicharacter $\mathrm{W}^{G} \in \mathcal{U}\left(\mathrm{C}_{0}(\hat{G}) \otimes \mathrm{C}_{0}(G)\right)$ coincides with the usual Drinfeld double of $G$.

\section{Universal C*-Algebras of Drinfeld Doubles and their Universal Property}

Let $G$ and $H$ be locally compact quantum groups and let $\mathrm{V} \in \mathcal{U}\left(\mathrm{C}_{0}(\hat{G}) \otimes \mathrm{C}_{0}(\hat{H})\right)$ be a bicharacter. Recall that the dual of the generalized Drinfeld double $\mathfrak{D}_{\mathrm{V}}$ is the quantum codouble $\widehat{\mathfrak{D}}_{\mathrm{V}}$ defined by

$$
\mathrm{C}_{0}\left(\widehat{\mathfrak{D}}_{\mathrm{V}}\right):=\mathrm{C}_{0}(\hat{H}) \otimes \mathrm{C}_{0}(\hat{G}),
$$

$\Delta_{\widehat{D}_{\mathrm{V}}}(\hat{y} \otimes \hat{x}):=\mathrm{V}_{23} \sigma_{23}\left(\Delta_{\hat{H}}(\hat{y}) \otimes \Delta_{\hat{G}}(\hat{x})\right) \mathrm{V}_{23}^{*} \quad$ for all $\hat{x} \in \mathrm{C}_{0}(\hat{G}), \hat{y} \in \mathrm{C}_{0}(\hat{H})$.

The goal of this section is to prove the existence of a coaction of $C_{0}^{\mathrm{u}}\left(\mathfrak{D}_{\mathrm{V}}\right)$ on $A \bigotimes_{\mathrm{V}} B$ (Lemma 3.4) satisfying a universal property formalized in Theorem 3.5. Let us start by proving the existence of a V-Drinfeld pair at the universal level. 
Proposition 3.1. There exists a unique pair of morphisms $\rho^{\mathrm{u}} \in \operatorname{Mor}\left(\mathrm{C}_{0}^{\mathrm{u}}(G), \mathrm{C}_{0}^{\mathrm{u}}\left(\mathfrak{D}_{\mathrm{V}}\right)\right)$ and $\theta^{\mathrm{u}} \in \operatorname{Mor}\left(\mathrm{C}_{0}^{\mathrm{u}}(H), \mathrm{C}_{0}^{\mathrm{u}}\left(\mathfrak{D}_{\mathrm{V}}\right)\right)$ satisfying the following commutation relation:

$$
\mathrm{V}_{12} \mathrm{~W}_{1 \rho^{\mathrm{u}}}^{G} \mathrm{~W}_{2 \theta^{\mathrm{u}}}^{H}=\mathrm{W}_{2 \theta^{\mathrm{u}}}^{H} \mathrm{~W}_{1 \rho^{\mathrm{u}}}^{G} \mathrm{~V}_{12} \quad \operatorname{in} \mathcal{U}\left(\mathrm{C}_{0}(\hat{G}) \otimes \mathrm{C}_{0}(\hat{H}) \otimes \mathrm{C}_{0}^{\mathrm{u}}\left(\mathfrak{D}_{\mathrm{V}}\right)\right) .
$$

Proof. Recall the universal representation $\mathrm{W}^{\mathfrak{D}_{\mathrm{V}}} \in \mathcal{U}\left(\mathrm{C}_{0}(\hat{H}) \otimes \mathrm{C}_{0}(\hat{G}) \otimes \mathrm{C}_{0}^{\mathrm{u}}\left(\mathfrak{D}_{\mathrm{V}}\right)\right)$ of $\widehat{\mathfrak{D}}_{\mathrm{V}}$ in $\mathrm{C}_{0}^{\mathrm{u}}\left(\mathfrak{D}_{\mathrm{V}}\right)$. By [39, Proposition 7.9], there exist representations $\mathrm{U}^{1} \in \mathcal{U}\left(\mathrm{C}_{0}(\hat{G}) \otimes\right.$ $\left.\mathrm{C}_{0}^{\mathrm{u}}\left(\mathfrak{D}_{\mathrm{V}}\right)\right)$ and $\mathrm{U}^{2} \in \mathcal{U}\left(\mathrm{C}_{0}(\hat{H}) \otimes \mathrm{C}_{0}^{\mathrm{u}}\left(\mathfrak{D}_{\mathrm{V}}\right)\right)$, respectively of $\hat{G}$ and $\hat{H}$ in $\mathrm{C}_{0}^{\mathrm{u}}\left(\mathfrak{D}_{\mathrm{V}}\right)$ such that

$$
\mathrm{W}^{\mathfrak{D} \mathrm{V}}=\mathrm{U}_{23}^{1} \mathrm{U}_{13}^{2} \text { and } \mathrm{V}_{12} \mathrm{U}_{13}^{1} \mathrm{U}_{23}^{2}=\mathrm{U}_{23}^{2} \mathrm{U}_{13}^{1} \mathrm{~V}_{12} \text {. }
$$

Universality of $\mathrm{W}^{G}$ and $\mathrm{W}^{H}$ gives unique $\mathrm{C}^{*}$-algebra morphisms $\rho^{\mathrm{u}} \in \operatorname{Mor}\left(\mathrm{C}_{0}^{\mathrm{u}}(G)\right.$, $\left.\mathrm{C}_{0}^{\mathrm{u}}\left(\mathfrak{D}_{\mathrm{V}}\right)\right)$ and $\theta^{\mathrm{u}} \in \operatorname{Mor}\left(\mathrm{C}_{0}^{\mathrm{u}}(H), \mathrm{C}_{0}^{\mathrm{u}}\left(\mathfrak{D}_{\mathrm{V}}\right)\right)$ such that $\left(\mathrm{id}_{\hat{G}} \otimes \rho^{\mathrm{u}}\right)\left(\mathrm{W}^{G}\right)=\mathrm{U}^{1}$ and $\left(\mathrm{id}_{\hat{H}} \otimes\right.$ $\left.\theta^{\mathrm{u}}\right)\left(\mathrm{W}^{H}\right)=\mathrm{U}^{2}$.

Therefore, we have

$$
\begin{aligned}
\mathrm{C}_{0}^{\mathrm{u}}\left(\mathfrak{D}_{\mathrm{V}}\right) & =\left\{\left(\omega_{1} \otimes \omega_{2} \otimes \mathrm{id}_{\mathfrak{D}_{\mathrm{V}}}\right)\left(\mathrm{W}^{\mathfrak{D}_{\mathrm{V}}}\right) \mid \omega_{1} \in \mathrm{C}_{0}(\hat{H})^{\prime}, \omega_{2} \in \mathrm{C}_{0}(\hat{G})^{\prime}\right\}^{\mathrm{CLS}} \\
& =\left\{\theta^{\mathrm{u}}\left(\left(\omega_{2} \otimes \mathrm{id}_{H}\right)\left(\mathrm{W}^{H}\right)\right) \rho^{\mathrm{u}}\left(\left(\omega_{1} \otimes \mathrm{id}_{G}\right)\left(\mathrm{W}^{G}\right)\right) \mid \omega_{1} \in \mathrm{C}_{0}(\hat{H})^{\prime}, \omega_{2} \in \mathrm{C}_{0}(\hat{G})^{\prime}\right\}^{\mathrm{CLS}} \\
& =\rho^{\mathrm{u}}\left(\mathrm{C}_{0}^{\mathrm{u}}(G)\right) \theta^{\mathrm{u}}\left(\mathrm{C}_{0}^{\mathrm{u}}(H)\right) .
\end{aligned}
$$

Since $\mathrm{W}^{G}, \mathrm{~W}^{H}, \mathrm{~W}^{\mathfrak{D}_{\mathrm{V}}}$ are bicharacters (see Example 2.9), we also obtain

$$
\begin{aligned}
& \left(\mathrm{id}_{\hat{H}} \otimes \mathrm{id}_{\hat{G}} \otimes \Delta_{\mathfrak{D}_{\mathrm{V}}}^{\mathrm{u}}\right)\left(\mathrm{W}^{\mathfrak{D}_{\mathrm{V}}}\right) \\
& \quad=\mathrm{W}_{123}^{\mathfrak{D}_{\mathrm{V}} \mathrm{W}_{124}^{\mathfrak{D}_{\mathrm{V}}}} \\
& \quad=\left(\mathrm{W}_{1 \rho^{\mathrm{u}}}^{G}\right)_{23}\left(\mathrm{~W}_{1 \theta^{\mathrm{u}}}^{H}\right)_{13}\left(\mathrm{~W}_{1 \rho^{\mathrm{u}}}^{G}\right)_{24}\left(\mathrm{~W}_{1 \theta^{\mathrm{u}}}^{H}\right)_{14} \\
& \quad=\left(\mathrm{W}_{1 \rho^{\mathrm{u}}}^{G}\right)_{23}\left(\mathrm{~W}_{1 \rho^{\mathrm{u}}}^{G}\right)_{24}\left(\mathrm{~W}_{1 \theta^{\mathrm{u}}}^{H}\right)_{13}\left(\mathrm{~W}_{1 \theta^{\mathrm{u}}}^{H}\right)_{14} \\
& \quad=\left(\left(\mathrm{id}_{\hat{G}} \otimes\left(\rho^{\mathrm{u}} \otimes \rho^{\mathrm{u}}\right) \circ \Delta_{G}^{\mathrm{u}}\right)\left(\mathrm{W}^{G}\right)\right)_{234}\left(\left(\mathrm{id}_{\hat{H}} \otimes\left(\theta^{\mathrm{u}} \otimes \theta^{\mathrm{u}}\right) \circ \Delta_{H}^{\mathrm{u}}\right)\left(\mathrm{W}^{H}\right)\right)_{134} .
\end{aligned}
$$

Taking the slice on the first and second leg by $\omega_{1} \otimes \omega_{2}$ for all $\omega_{1} \in \mathrm{C}_{0}(\hat{H})^{\prime}, \omega_{2} \in \mathrm{C}_{0}(\hat{G})^{\prime}$ and using the first equality in (3.2) we obtain

$$
\Delta_{\mathfrak{D}_{\mathrm{V}}}^{\mathrm{u}}\left(\rho^{\mathrm{u}}(x) \theta^{\mathrm{u}}(y)\right)=\left(\rho^{\mathrm{u}} \otimes \rho^{\mathrm{u}}\right)\left(\Delta_{G}^{\mathrm{u}}(x)\right)\left(\theta^{\mathrm{u}} \otimes \theta^{\mathrm{u}}\right)\left(\Delta_{H}^{\mathrm{u}}(y)\right)
$$

for all $x \in \mathrm{C}_{0}^{\mathrm{u}}(G), y \in \mathrm{C}_{0}^{\mathrm{u}}(H)$.

Definition 3.2. The pair of morphisms $\left(\rho^{\mathrm{u}}, \theta^{\mathrm{u}}\right)$ is called the universal $\mathrm{V}$-Drinfeld pair.

Lemma 3.3. The maps $\rho^{\mathrm{u}}$ and $\theta^{\mathrm{u}}$ are Hopf ${ }^{*}$-homomorphisms. Furthermore $\Lambda_{\mathfrak{D}_{\mathrm{V}}} \circ \rho^{\mathrm{u}}=$ $\rho \circ \Lambda_{G}$ and $\Lambda_{\mathfrak{D}_{\mathrm{V}}} \circ \theta^{\mathrm{u}}=\theta \circ \Lambda_{H}$.

Proof. The reduced bicharacter of $\mathfrak{D}_{\mathrm{V}}$ is $\mathrm{W}^{\mathfrak{D}_{\mathrm{V}}}=\mathrm{W}_{2 \theta}^{H} \mathrm{~W}_{1 \rho}^{G} \in \mathcal{U}\left(\mathrm{C}_{0}(\hat{H}) \otimes \mathrm{C}_{0}(\hat{G}) \otimes\right.$ $\mathrm{C}_{0}\left(\mathfrak{D}_{\mathrm{V}}\right)$ ) (see [39, Theorem 5.1(iii)]). Using the first equality in (3.2) we have

$$
\begin{aligned}
\mathrm{W}^{\mathfrak{D}_{\mathrm{V}}} & =\left(\mathrm{id}_{\hat{G}} \otimes \Lambda_{\mathfrak{D}_{\mathrm{V}}}\right)\left(\mathrm{W}^{\mathfrak{D}_{\mathrm{V}}}\right) \\
& \left.=\left(\left(\mathrm{id}_{\hat{G}} \otimes \theta\right)\left(\mathrm{W}^{H}\right)\right)_{23}\left(\mathrm{id}_{\hat{G}} \otimes \rho\right)\left(\mathrm{W}^{G}\right)\right)_{13} \\
& =\left(\operatorname{id}_{\hat{H}} \otimes \mathrm{id}_{\hat{G}} \otimes \Lambda_{\mathfrak{D}_{\mathrm{V}}}\right)\left(\mathrm{W}^{\mathfrak{D} \mathrm{V}}\right) \\
& =\left(\left(\mathrm{id}_{\hat{H}} \otimes \Lambda_{\mathfrak{D}_{\mathrm{V}}} \circ \theta^{\mathrm{u}}\right)\left(\mathrm{W}^{H}\right)\right)_{23}\left(\left(\mathrm{id}_{\hat{G}} \otimes \Lambda_{\mathfrak{D}_{\mathrm{V}}} \circ \rho^{\mathrm{u}}\right)\left(\mathrm{W}^{G}\right)\right)_{13}
\end{aligned}
$$


in $\mathcal{U}\left(\mathrm{C}_{0}(\hat{H}) \otimes \mathrm{C}_{0}(\hat{G}) \otimes \mathrm{C}_{0}\left(\mathfrak{D}_{\mathrm{V}}\right)\right)$. The last equation implies

$$
\begin{aligned}
& \left(\left(\operatorname{id}_{\hat{H}} \otimes \Lambda_{\mathfrak{D}_{\mathrm{V}}} \circ \theta^{\mathrm{u}}\right)\left(\mathrm{W}^{H}\right)\right)_{23}^{*}\left(\left(\mathrm{id}_{\hat{G}} \otimes \theta\right)\left(\mathrm{W}^{H}\right)\right)_{23} \\
& \left.\quad=\left(\left(\mathrm{id}_{\hat{G}} \otimes \Lambda_{\mathfrak{D}_{\mathrm{V}}} \circ \rho^{\mathrm{u}}\right)\left(\mathrm{W}^{G}\right)\right)_{13}\left(\mathrm{id}_{\hat{G}} \otimes \rho\right)\left(\mathrm{W}^{G}\right)\right)_{13}^{*} .
\end{aligned}
$$

Clearly, the first leg in the term of the left hand side and the second leg in the term of the right hand side of the above equation are trivial. Hence, both the terms are equal to $1_{\hat{H}} \otimes 1_{\hat{G}} \otimes u$ for some element $u \in \mathcal{U}\left(\mathrm{C}_{0}\left(\mathfrak{D}_{\mathrm{V}}\right)\right)$. In particular, we have

$$
\left(\operatorname{id}_{\hat{G}} \otimes \Lambda_{\mathfrak{D}_{\mathrm{V}}} \circ \rho^{\mathrm{u}}\right)\left(\mathrm{W}^{G}\right)=\left(1_{\hat{G}} \otimes u\right)\left(\mathrm{id}_{\hat{G}} \otimes \rho\right)\left(\mathrm{W}^{G}\right) \quad \text { in } \mathcal{U}\left(\mathrm{C}_{0}(\hat{G}) \otimes \mathrm{C}_{0}\left(\mathfrak{D}_{\mathrm{V}}\right)\right)
$$

Since $\mathrm{W}^{G}$ is the (universal) left representation of $\hat{G}$ in $\mathrm{C}_{0}^{\mathrm{u}}(G)$, the unitary $\left(\left(\mathrm{id}_{\hat{G}} \otimes\right.\right.$ $\left.\Lambda_{\mathfrak{D}_{\mathrm{V}}} \circ \rho^{\mathrm{u}}\right)\left(\mathrm{W}^{G}\right)$ is also a representation of $\hat{G}$ in $\mathrm{C}_{0}\left(\mathfrak{D}_{\mathrm{V}}\right)$. Also, $\left(\operatorname{id}_{\hat{G}} \otimes \rho\right)\left(\mathrm{W}^{G}\right)$ is a left representation of $\hat{G}$ in $\mathrm{C}_{0}\left(\mathfrak{D}_{\mathrm{V}}\right)$. These facts force $u=1_{\mathfrak{D}_{\mathrm{V}}}$. Now $\left(\operatorname{id}_{\hat{G}} \otimes \rho\right)\left(\mathrm{W}^{G}\right)$ is the bicharacter induced by the Hopf*-homomorphism $\rho$. Then (3.3) and (2.16) show that $\rho^{\mathrm{u}}$ is a Hopf ${ }^{*}$-homomorphism which is the unique universal lift of $\rho$. Slicing the first leg of the both sides of (3.3) with $\omega \in \mathrm{C}_{0}(\hat{G})^{\prime}$ we get $\Lambda_{\mathfrak{D}_{\mathrm{V}}} \circ \rho^{\mathrm{u}}=\rho \circ \Lambda_{G}$. The rest of the proof follows in a similar way.

3.1. Coaction on the twisted tensor products. As a prelude to the results proved in the next section, we show that there is a unique coaction $\gamma_{A}^{\mathrm{u}} \bowtie_{\mathrm{V}} \gamma_{B}^{\mathrm{u}} \in \operatorname{Mor}\left(A \bigotimes_{\mathrm{V}} B, A \bigotimes_{\mathrm{V}}\right.$ $\left.B \otimes \mathrm{C}_{0}^{\mathrm{u}}\left(\mathfrak{D}_{\mathrm{V}}\right)\right)$ satisfying some natural conditions.

Lemma 3.4. Let $\left(A, \gamma_{A}\right)$ and $\left(B, \gamma_{B}\right)$ be $G$ - and $H-C^{*}$-algebras such that $\gamma_{A}$ and $\gamma_{B}$ are injective. Let $\gamma_{A}^{\mathrm{u}}$ and $\gamma_{B}^{\mathrm{u}}$ be the respective universal normal coactions. Then there is a unique coaction $\gamma_{A}^{\mathrm{u}} \bowtie \gamma_{\mathrm{V}} \gamma_{B}^{\mathrm{u}} \in \operatorname{Mor}\left(A \bigotimes_{\mathrm{V}} B, A \bigotimes_{\mathrm{V}} B \otimes \mathrm{C}_{0}^{\mathrm{u}}\left(\mathfrak{D}_{\mathrm{V}}\right)\right)$ such that

$$
\gamma_{A}^{\mathrm{u}} \bowtie \mathrm{V} \gamma_{B}^{\mathrm{u}}\left(j_{A}(a)\right)=\left(j_{A} \otimes \rho^{\mathrm{u}}\right)\left(\gamma_{A}^{\mathrm{u}}(a)\right), \quad \gamma_{B}^{\mathrm{u}} \bowtie \mathrm{V} \gamma_{B}^{\mathrm{u}}\left(j_{B}(b)\right)=\left(j_{B} \otimes \theta^{\mathrm{u}}\right)\left(\gamma_{B}^{\mathrm{u}}(b)\right)
$$

for all $a \in A, b \in B$.

Proof. Since $\gamma_{A}$ and $\gamma_{B}$ are injective, the canonical coaction $\gamma_{A} \bowtie_{\mathrm{V}} \gamma_{B}$ defined by (2.24) is injective by Lemma 2.14. Hence, there is a unique normal coaction of $C_{0}^{u}\left(\mathfrak{D}_{\mathrm{V}}\right)$, denoted by $\gamma_{A}^{\mathrm{u}} \bowtie_{\mathrm{V}} \gamma_{B}^{\mathrm{u}}$, such that

$$
\left(\operatorname{id}_{A \bigotimes_{\mathrm{V} B}} \otimes \Lambda_{\mathfrak{D}_{\mathrm{V}}}\right) \circ \gamma_{A}^{\mathrm{u}} \bowtie_{\mathrm{V}} \gamma_{B}^{\mathrm{u}}=\gamma_{A} \bowtie_{\mathrm{V}} \gamma_{B}
$$

Therefore, by (2.24), for $a \in A$ we have

$$
\left(\operatorname{id}_{A \nabla_{\mathrm{V} B}} \otimes \Lambda_{\mathfrak{D}_{\mathrm{V}}}\right)\left(\gamma_{A}^{\mathrm{u}} \bowtie \mathrm{V} \gamma_{B}^{\mathrm{u}}\left(j_{A}(a)\right)\right)=\gamma_{A} \bowtie \gamma_{\mathrm{V}}\left(j_{A}(a)\right)=\left(j_{A} \otimes \rho\right)\left(\gamma_{A}(a)\right)
$$

where the last equality uses Lemma 3.3 and the definition of induced coactions.

Let $\Delta_{\mathfrak{D}_{\mathrm{V}}}^{\mathrm{r}, \mathrm{u}} \in \operatorname{Mor}\left(\mathrm{C}_{0}\left(\mathfrak{D}_{\mathrm{V}}\right), \mathrm{C}_{0}\left(\mathfrak{D}_{\mathrm{V}}\right) \otimes \mathrm{C}_{0}^{\mathrm{u}}\left(\mathfrak{D}_{\mathrm{V}}\right)\right)$ denote the universal lift of $\Delta_{\mathfrak{D}_{\mathrm{V}}}$ while viewed as coaction of $\mathrm{C}_{0}\left(\mathfrak{D}_{\mathrm{V}}\right)$ on $\mathrm{C}_{0}\left(\mathfrak{D}_{\mathrm{V}}\right)$. By virtue of [40, Proposition 4.8], $\Delta_{\mathfrak{D}_{\mathrm{V}}}^{\mathrm{r}, \mathrm{u}}$ satisfies the following condition

$$
\left(\Lambda_{\mathfrak{D}_{\mathrm{V}}} \otimes \mathrm{id}_{\mathfrak{D}_{\mathrm{V}}}\right) \circ \Delta_{\mathfrak{D}_{\mathrm{V}}}^{\mathrm{u}}=\Delta_{\mathfrak{D}_{\mathrm{V}}}^{\mathrm{r}, \mathrm{u}} \circ \Lambda_{\mathfrak{D}_{\mathrm{V}}}
$$


By (2.22) and repeated application of (3.5), (2.24) and Lemma 3.3 gives

$$
\begin{aligned}
& \left(\gamma_{A} \bowtie \mathrm{V} \gamma_{B} \otimes \operatorname{id}_{\mathfrak{D}_{\mathrm{V}}^{\mathrm{u}}}\right)\left(\gamma_{A}^{\mathrm{u}} \bowtie \mathrm{V} \gamma_{B}^{\mathrm{u}}\left(j_{A}(a)\right)\right) \\
& =\left(\mathrm{id}_{A \otimes B} \otimes \Lambda_{\mathfrak{D}_{\mathrm{V}}} \otimes \mathrm{id}_{\mathfrak{D}_{\mathrm{V}}}\right)\left(\left(\gamma_{A}^{\mathrm{u}} \bowtie \mathrm{V} \gamma_{B}^{\mathrm{u}} \otimes \mathrm{id}_{\mathfrak{D}_{\mathrm{V}}}\right)\left(\gamma_{A}^{\mathrm{u}} \bowtie_{\mathrm{V}} \gamma_{B}^{\mathrm{u}}\left(j_{A}(a)\right)\right)\right) \\
& =\left(\mathrm{id}_{A \otimes B} \otimes\left(\Lambda_{\mathfrak{D}_{\mathrm{V}}^{\mathrm{u}}} \otimes \mathrm{id}_{\mathfrak{D}_{\mathrm{V}}}\right) \circ \Delta_{\mathfrak{D}_{\mathrm{V}}}^{\mathrm{u}}\right)\left(\gamma_{A}^{\mathrm{u}} \bowtie \mathrm{V} \gamma_{B}^{\mathrm{u}}\left(j_{A}(a)\right)\right) \\
& =\left(\operatorname{id}_{A \otimes B} \otimes \Delta_{\mathfrak{D}_{\mathrm{V}}}^{\mathrm{r}, \mathrm{u}}\right)\left(\gamma_{A} \bowtie \mathrm{V} \gamma_{B}\left(j_{A}(a)\right)\right) \\
& =\left(\operatorname{id}_{A \otimes B} \otimes \Delta_{\mathfrak{D}_{\mathrm{V}}}^{\mathrm{r}, \mathrm{u}}\right)\left(\left(j_{A} \otimes \rho\right)\left(\gamma_{A}(a)\right)\right. \\
& =\left(\operatorname{id}_{A \otimes B} \otimes \Delta_{\mathfrak{D}_{\mathrm{V}}}^{\mathrm{r}, \mathrm{u}} \circ \Lambda_{\mathfrak{D}_{\mathrm{V}}}\right)\left(\left(j_{A} \otimes \rho^{\mathrm{u}}\right) \gamma_{A}^{\mathrm{u}}(a)\right) \\
& =\left(\mathrm{id}_{A \otimes B} \otimes\left(\Lambda_{\mathfrak{D}_{\mathrm{V}}} \otimes \mathrm{id}_{\mathfrak{D}_{\mathrm{V}}}\right) \circ \Delta_{\mathfrak{D}_{\mathrm{V}}}^{\mathrm{u}} \circ \rho^{\mathrm{u}}\right)\left(\left(j_{A} \otimes \operatorname{id}_{\mathrm{C}_{0}\left(G^{\mathrm{u}}\right)}\right) \gamma_{A}^{\mathrm{u}}(a)\right) \\
& =\left(j_{A} \otimes \Lambda_{\mathfrak{D}_{\mathrm{V}}} \circ \rho^{\mathrm{u}} \otimes \rho^{\mathrm{u}}\right)\left(\left(\mathrm{id}_{A} \otimes \Delta_{G^{\mathrm{u}}}\right)\left(\gamma_{A}^{\mathrm{u}}(a)\right)\right) \\
& =\left(\left(j_{A} \otimes \rho \circ \Lambda_{G}\right) \circ \gamma_{A}^{\mathrm{u}} \otimes \rho^{\mathrm{u}}\right)\left(\gamma_{A}^{\mathrm{u}}(a)\right) \\
& =\left(\left(j_{A} \otimes \rho\right) \gamma_{A} \otimes \rho^{\mathrm{u}}\right)\left(\gamma_{A}^{\mathrm{u}}(a)\right)=\left(\gamma_{A} \bowtie \mathrm{V} \gamma_{B} \otimes \mathrm{id}_{\mathfrak{D}_{\mathrm{V}}^{\mathrm{u}}}\right)\left(\left(j_{A} \otimes \rho^{\mathrm{u}}\right)\left(\gamma_{A}^{\mathrm{u}}(a)\right)\right) \text {. }
\end{aligned}
$$

Now, injectivity of $\gamma_{A} \bowtie \mathrm{V} \gamma_{B}$ gives $\gamma_{A}^{\mathrm{u}} \bowtie \mathrm{V} \gamma_{B}^{\mathrm{u}}\left(j_{A}(a)\right)=\left(j_{A} \otimes \rho^{\mathrm{u}}\right) \gamma_{A}^{\mathrm{u}}(a)$ for all $a \in A$. The second part of (3.4) can be shown similarly.

3.2. Universal property of $\mathrm{C}_{0}^{\mathrm{u}}\left(\mathfrak{D}_{\mathrm{V}}\right)$. Let $\left(\gamma_{A}^{\mathrm{u}}, A\right)$ be a coaction of $\mathrm{C}_{0}^{\mathrm{u}}(G)$ on a $\mathrm{C}^{*}$-algebra $A$; in other words $\gamma_{A}^{\mathrm{u}}$ is an action of $G$ on the universal level.

In the spirit of [22, Definition 4.2], we say that the action $\gamma_{A}^{\mathrm{u}}$ is faithful if the *-algebra generated by the set $\left\{\left(\omega_{1} \otimes \operatorname{id}_{G^{u}}\right)\left(\gamma_{A}^{\mathrm{u}}(a)\right) \mid \omega_{1} \in A^{\prime}, a \in A\right\}$ is strictly dense in $\mathcal{M}\left(\mathrm{C}_{0}^{\mathrm{u}}(G)\right)$. If $G$ is compact then we get the usual definition of faithfulness of the action $\gamma_{A}^{\mathrm{u}}$ : ${ }^{*}$-algebra generated by the set $\left\{\left(\omega_{1} \otimes \operatorname{id}_{G}\right)\left(\gamma_{A}^{\mathrm{u}}(a)\right) \mid \omega_{1} \in A^{\prime}, a \in A\right\}$ is norm dense in $\mathrm{C}^{\mathrm{u}}(G)$.

Theorem 3.5. Let $G, H$ be locally compact quantum groups and let $A, B$ be $\mathrm{C}^{*}$-algebras. Let $\left(A, \gamma_{A}^{\mathrm{u}}\right),\left(B, \gamma_{B}^{\mathrm{u}}\right)$, be an action of $G$ on $A$ on the universal level, respectively an action of $H$ on $B$ on the universal level. Assume that $\gamma_{A}^{\mathrm{u}}$ and $\gamma_{B}^{\mathrm{u}}$ are faithful and normal.

Suppose that $I$ is a locally compact quantum group and

(1) there is a coaction $\gamma \in \operatorname{Mor}\left(A \nabla_{\mathrm{V}} B, A \bigotimes_{\mathrm{V}} B \otimes \mathrm{C}_{0}^{\mathrm{u}}(I)\right)$ of $\mathrm{C}_{0}^{\mathrm{u}}(I)$ on $A \bigotimes_{\mathrm{V}} B$;

(2) there are Hopf ${ }^{*}$-homomorphisms $\rho_{1} \in \operatorname{Mor}\left(\mathrm{C}_{0}^{\mathrm{u}}(G), \mathrm{C}_{0}^{\mathrm{u}}(I)\right)$ and $\theta_{1} \in \operatorname{Mor}\left(\mathrm{C}_{0}^{\mathrm{u}}(H)\right.$, $\left.\mathrm{C}_{0}^{\mathrm{u}}(I)\right)$ such that

$$
\gamma \circ j_{A}=\left(j_{A} \otimes \rho_{1}\right) \circ \gamma_{A}^{\mathrm{u}}, \quad \gamma \circ j_{B}=\left(j_{B} \otimes \theta_{1}\right) \circ \gamma_{B}^{\mathrm{u}} .
$$

Then there is a unique Hopf ${ }^{*}$-homomorphism $\Psi \in \operatorname{Mor}\left(\mathrm{C}_{0}^{\mathrm{u}}\left(\mathfrak{D}_{\mathrm{V}}\right), \mathrm{C}_{0}^{\mathrm{u}}(I)\right)$ such that $\Psi$ 。 $\rho^{\mathrm{u}}=\rho_{1}$ and $\Psi \circ \theta^{\mathrm{u}}=\theta_{1}$. In particular, this implies that $\left(\mathrm{id}_{A \bigotimes_{\mathrm{V}} B} \otimes \Psi\right) \circ \gamma_{A}^{\mathrm{u}} \bowtie \gamma_{B}^{\mathrm{u}} \circ j_{A}=$ $\gamma \circ j_{A}$ and $\left(\mathrm{id}_{A \otimes_{\mathrm{V} B}} \otimes \Psi\right) \circ \gamma_{A}^{\mathrm{u}} \bowtie \gamma_{B}^{\mathrm{u}} \circ j_{B}=\gamma \circ j_{B}$.

Proof. Denote the induced coactions of $\mathrm{C}_{0}(G)$ and $\mathrm{C}_{0}(H)$ on $A$ and $B$ by $\gamma_{A}$ and $\gamma_{B}$, respectively. Since $\gamma_{A}^{\mathrm{u}}$ and $\gamma_{B}^{\mathrm{u}}$ are normal, $\gamma_{A}$ and $\gamma_{B}$ are injective.

Let $(\alpha, \beta)$ be a V-Heisenberg pair on a Hilbert space $\mathcal{H}$. Then using the definition of $j_{A}$ we compute $(a \in A)$

$$
\begin{aligned}
\gamma\left(j_{A}(a)\right)=\left(j_{A} \otimes \rho_{1}\right)\left(\gamma_{A}^{\mathrm{u}}(a)\right) & =\left(\left(\left(\mathrm{id}_{A} \otimes \alpha\right) \gamma_{A} \otimes \rho_{1}\right)\left(\gamma_{A}^{\mathrm{u}}(a)\right)\right)_{134} \\
& =\left(\left(\left(\mathrm{id}_{A} \otimes \alpha \circ \Lambda_{G}\right) \gamma_{A}^{\mathrm{u}} \otimes \rho_{1}\right)\left(\gamma_{A}^{\mathrm{u}}(a)\right)\right)_{134} \\
& =\left(\left(\left(\mathrm{id}_{A} \otimes \alpha \circ \Lambda_{G} \otimes \rho_{1}\right)\left(\mathrm{id}_{A} \otimes \Delta_{G}^{\mathrm{u}}\right)\left(\gamma_{A}^{\mathrm{u}}(a)\right)\right)_{134}\right.
\end{aligned}
$$


Similarly, we obtain

$$
\gamma\left(j_{B}(b)\right)=\left(\left(\left(\operatorname{id}_{B} \otimes \beta \circ \Lambda_{H} \otimes \theta_{1}\right)\left(\left(\operatorname{id}_{B} \otimes \Delta_{H}^{\mathrm{u}}\right)\left(\gamma_{B}^{\mathrm{u}}(b)\right)\right)\right)_{234} .\right.
$$

Let $(\bar{\alpha}, \bar{\beta})$ be a V-anti-Heisenberg pair on a Hilbert space $\mathcal{H}_{1}$ (see [35, Lemmas 3.6 and 3.8] for its existence):

$$
\mathrm{W}_{2 \bar{\beta}}^{H} \mathrm{~W}_{1 \bar{\alpha}}^{G}=\mathrm{V}_{12} \mathrm{~W}_{1 \bar{\alpha}}^{G} \mathrm{~W}_{2 \bar{\beta}}^{H} \quad \text { in } \mathcal{U}\left(\mathrm{C}_{0}(\hat{G}) \otimes \mathrm{C}_{0}(\hat{H}) \otimes \mathbb{K}\left(\mathcal{H}_{1}\right)\right) .
$$

By [40, Proposition 5.10], $j_{A}$ and $j_{B}$ satisfy the following commutation relation:

$$
\left[\left(j_{A} \otimes \bar{\alpha}\right)\left(\gamma_{A}(a)\right),\left(j_{B} \otimes \bar{\beta}\right)\left(\gamma_{B}(b)\right)\right]=0 \quad \text { for all } a \in A, b \in B .
$$

This implies that

$$
\left[\left(\gamma \circ j_{A} \otimes \bar{\alpha}\right)\left(\gamma_{A}(a)\right),\left(\gamma \circ j_{B} \otimes \bar{\beta}\right)\left(\gamma_{B}(b)\right)\right]=0 \quad \text { for all } a \in A, b \in B .
$$

This is in turn equivalent to

$$
\begin{aligned}
& {\left[\left(\left(\operatorname{id}_{A} \otimes \alpha \circ \Lambda_{G} \otimes \rho_{1} \otimes \Lambda_{G} \circ \bar{\alpha}\right)\left(\operatorname{id}_{A} \otimes\left(\operatorname{id}_{G} \otimes \Delta_{G^{\mathrm{u}}}\right) \circ \Delta_{G}^{\mathrm{u}}\right)\left(\gamma_{A}^{\mathrm{u}}(a)\right)\right)_{1345},\right.} \\
& \left.\left(\left(\mathrm{id}_{B} \otimes \beta \circ \Lambda_{H} \otimes \theta_{1} \otimes \Lambda_{H} \circ \bar{\beta}\right)\left(\mathrm{id}_{B} \otimes\left(\mathrm{id}_{H} \otimes \Delta_{H^{\mathrm{u}}}\right) \circ \Delta_{H}^{\mathrm{u}}\right)\left(\gamma_{B}^{\mathrm{u}}(b)\right)\right)_{2345}\right]=0 .
\end{aligned}
$$

Therefore, the following operators

$$
\begin{aligned}
& \alpha^{\prime}(x):=\left(\alpha \circ \Lambda_{G} \otimes \rho_{1} \otimes \Lambda_{G} \circ \bar{\alpha}\right)\left(\left(\operatorname{id}_{G} \otimes \Delta_{G}^{\mathrm{u}}\right)\left(\Delta_{G}^{\mathrm{u}}(x)\right)\right), \\
& \beta^{\prime}(y):=\left(\beta \circ \Lambda_{H} \otimes \theta_{1} \otimes \Lambda_{H} \circ \bar{\beta}\right)\left(\left(\operatorname{id}_{H} \otimes \Delta_{H}^{\mathrm{u}}\right)\left(\Delta_{H}^{\mathrm{u}}(y)\right)\right)
\end{aligned}
$$

commute for all $x=\left(\omega_{1} \otimes \operatorname{id}_{G}\right)\left(\gamma_{A}^{\mathrm{u}}(a)\right) \in \mathcal{M}\left(\mathrm{C}_{0}^{\mathrm{u}}(G)\right), y=\left(\omega_{2} \otimes \operatorname{id}_{H}\right)\left(\gamma_{B}^{\mathrm{u}}(b)\right) \in$ $\mathcal{M}\left(\mathrm{C}_{0}^{\mathrm{u}}(H)\right)$, where $\omega_{1} \in A^{\prime}, \omega_{2} \in B^{\prime}, a \in A, b \in B$. Now faithfulness of $\gamma_{A}^{\mathrm{u}}$ and $\gamma_{B}^{\mathrm{u}}$ shows that the above operators commute for all $x \in \mathcal{M}\left(\mathrm{C}_{0}^{\mathrm{u}}(G)\right)$ and $y \in \mathcal{M}\left(\mathrm{C}_{0}^{\mathrm{u}}(H)\right)$; hence, in particular for all $x \in \mathrm{C}_{0}^{\mathrm{u}}(G)$ and $y \in \mathrm{C}_{0}^{\mathrm{u}}(H)$.

Equivalently,

$$
\mathrm{W}_{1 \alpha^{\prime}}^{G} \mathrm{~W}_{2 \beta^{\prime}}^{H}=\mathrm{W}_{2 \beta^{\prime}}^{H} \mathrm{~W}_{1 \alpha^{\prime}}^{G} \quad \text { in } \mathcal{U}\left(\mathrm{C}_{0}(\hat{G}) \otimes \mathrm{C}_{0}(\hat{H}) \otimes \mathbb{K}(\mathcal{H}) \otimes \mathrm{C}_{0}^{\mathrm{u}}(I) \otimes \mathbb{K}\left(\mathcal{H}_{1}\right)\right) .
$$

Using the properties of $\mathrm{W}^{G}, \mathrm{~W}^{H}, \Lambda_{G}, \Lambda_{H}$ and the Eqs. (2.18), (3.6) we obtain:

$$
\begin{aligned}
\mathrm{W}_{1 \alpha^{\prime}}^{G} \mathrm{~W}_{2 \beta^{\prime}}^{H} & =\mathrm{W}_{1 \alpha}^{G} \mathrm{~W}_{1 \rho_{1}}^{G} \mathrm{~W}_{1 \bar{\alpha}}^{G} \mathrm{~W}_{2 \beta}^{H} \mathrm{~W}_{2 \theta_{1}}^{H} \mathrm{~W}_{2 \bar{\beta}}^{H} \\
& =\mathrm{W}_{1 \alpha}^{G} \mathrm{~W}_{2 \beta}^{H} \mathrm{~W}_{1 \rho_{1}}^{G} \mathrm{~W}_{2 \theta_{1}}^{H} \mathrm{~W}_{1 \bar{\alpha}}^{G} \mathrm{~W}_{2 \bar{\beta}}^{H} \\
& =\mathrm{W}_{2 \beta}^{H} \mathrm{~W}_{1 \alpha}^{G} \mathrm{~V}_{12} \mathrm{~W}_{1 \rho_{1}}^{G} \mathrm{~W}_{2 \theta_{1}}^{H} \mathrm{~V}_{12}^{*} \mathrm{~W}_{2 \bar{\beta}}^{H} \mathrm{~W}_{1 \bar{\alpha}}^{G} .
\end{aligned}
$$

In the computation above the morphisms $\alpha, \beta$ are acting on the third leg, $\rho_{1}, \theta_{1}$ are acting on the fourth leg and $\bar{\alpha}, \bar{\beta}$ are acting on the fifth leg, respectively.

Similarly, we get

$$
\mathrm{W}_{2 \beta^{\prime}} \mathrm{W}_{1 \alpha^{\prime}}^{G}=\mathrm{W}_{2 \beta}^{H} \mathrm{~W}_{1 \alpha}^{G} \mathrm{~W}_{2 \rho_{1}}^{H} \mathrm{~W}_{1 \rho_{1}}^{G} \mathrm{~W}_{2 \bar{\beta}}^{H} \mathrm{~W}_{1 \bar{\alpha}}^{G} .
$$

Then (3.7) gives

$$
\mathrm{V}_{12} \mathrm{~W}_{1 \rho_{1}}^{G} \mathrm{~W}_{2 \theta_{1}}^{H}=\mathrm{W}_{2 \rho_{1}}^{H} \mathrm{~W}_{1 \rho_{1}}^{G} \mathrm{~V}_{12} \quad \text { in } \mathcal{U}\left(\mathrm{C}_{0}(\hat{G}) \otimes \mathrm{C}_{0}(\hat{H}) \otimes \mathrm{C}_{0}^{\mathrm{u}}(I)\right) .
$$


By [39, Proposition 7.9], $\mathrm{W}_{1 \rho_{1}}^{G} \mathrm{~W}_{2 \theta_{1}}^{H}$ is a left representation of $\widehat{\mathfrak{D}}_{\mathrm{V}}$ in $\mathrm{C}_{0}^{\mathrm{u}}(I)$. Hence, there is a unique $\eta \in \operatorname{Mor}\left(\mathrm{C}_{0}^{\mathrm{u}}\left(\mathfrak{D}_{\mathrm{V}}\right), \mathrm{C}_{0}^{\mathrm{u}}(I)\right)$ such that $\left(\mathrm{id}_{\hat{G}} \otimes \operatorname{id}_{\hat{H}} \otimes \eta\right)\left(\mathrm{W}_{1 \rho^{\mathrm{u}}}^{G} \mathrm{~W}_{2 \theta^{\mathrm{u}}}^{H}\right)=$ $\mathrm{W}_{1 \rho_{1}}^{G} \mathrm{~W}_{2 \theta_{1}}^{H}$, which implies $\left(\left(\mathrm{id}_{\hat{G}} \otimes \eta\right)\left(\mathrm{W}_{1 \rho^{\mathrm{u}}}^{G}\right)\right)\left(\left(\mathrm{id}_{\hat{H}} \otimes \eta\right)\left(\mathrm{W}_{2 \theta^{\mathrm{u}}}^{H}\right)\right)=\mathrm{W}_{1 \rho_{1}}^{G} \mathrm{~W}_{2 \theta_{1}}^{H}$. Equivalently,

$$
\left(\mathrm{W}_{1 \rho_{1}}^{G}\right)^{*}\left(\left(\mathrm{id}_{\hat{G}} \otimes \eta\right)\left(\mathrm{W}_{1 \rho^{\mathrm{u}}}^{G}\right)\right)=\mathrm{W}_{2 \theta_{1}}^{H}\left(\left(\mathrm{id}_{\hat{H}} \otimes \eta\right)\left(\mathrm{W}_{2 \theta^{\mathrm{u}}}^{H}\right)\right)^{*}
$$

in $\mathcal{U}\left(\mathrm{C}_{0}(\hat{G}) \otimes \mathrm{C}_{0}(\hat{H}) \otimes \mathrm{C}_{0}^{\mathrm{u}}(I)\right)$. Hence, there is a unique $u \in \mathcal{U}\left(\mathrm{C}_{0}^{\mathrm{u}}(I)\right)$ such that $\left(\left(\mathrm{id}_{\hat{G}} \otimes\right.\right.$ $\left.\eta \circ \rho^{\mathrm{u}}\right)\left(\mathrm{W}^{G}\right)=\left(\mathrm{id}_{\hat{G}} \otimes \rho_{1}\right)\left(\mathrm{W}^{G}\right)(1 \otimes u)$ and $\left(\mathrm{id}_{H} \otimes \theta_{1}\right)\left(\mathrm{W}^{H}\right)=(1 \otimes u)\left(\left(\mathrm{id}_{\hat{H}} \otimes \eta \circ\right.\right.$ $\left.\theta^{\mathrm{u}}\right)\left(\mathrm{W}^{H}\right)$. Now $\left(\mathrm{id}_{H} \otimes \theta_{1}\right)\left(\mathrm{W}^{H}\right) \in \mathcal{U}\left(\mathrm{C}_{0}(\hat{G}) \otimes \mathrm{C}_{0}^{\mathrm{u}}\left(\mathfrak{D}_{\mathrm{V}}\right)\right)$ is a left representation of $\hat{G}$ in $\mathrm{C}_{0}^{\mathrm{u}}\left(\mathfrak{D}_{\mathrm{V}}\right)$. Repeating the argument used in the proof of Lemma 3.3 we can conclude that $u=1$ and this completes the proof.

\section{Orthogonal Filtrations of $C^{*}$-Algebras and their Quantum Symmetries}

The theory of quantum symmetry group of orthogonal filtrations first appeared in [8]. Later, M. Thibault de Chanvalon extended the theory to Hilbert modules in the paper [18]. In this section we recall the notions of orthogonal filtration of a unital $\mathrm{C}^{*}$-algebra and their quantum symmetry groups as developed in these two papers. Then, under suitable conditions, we prove the existence of a canonical filtration of twisted tensor products.

Definition 4.1 ([18], Definition 2.4). Let $A$ be a unital $C^{*}$-algebra and let $\tau_{A}$ be a faithful state on $A$. An orthogonal filtration for the pair $\left(A, \tau_{A}\right)$ is a sequence of finite dimensional subspaces $\left\{A_{i}\right\}_{i \geq 0}$ such that $A_{0}=\mathbb{C} 1_{A}$, Span $\cup_{i \geq 0} \underset{A_{i}}{A_{i}}$ is dense in $A$ and $\tau_{A}\left(a^{*} b\right)=0$ if $a \in A_{i}, b \in A_{j}$ and $i \neq j$. We will usually write $\widetilde{A}$ for the triple $\left(A, \tau_{A},\left\{A_{i}\right\}_{i \geq 0}\right)$ as above.

Remark 4.2. Thibault de Chanvalon's definition replaced subspaces $A_{i}$ by suitable Hilbert modules. However, if we view a unital $C^{*}$-algebra $A$ as a right Hilbert module over itself and we take $W=\mathbb{C} 1_{A}$ and $J$ to be the map $a \mapsto a^{*}$, then the above definition indeed coincides with the one given in Definition 2.4 of [18]. We should mention that in the original formulation of [8] it was additionally assumed that $\operatorname{Span} \cup_{i>0} A_{i}$ is a $*$-algebra; at the same time the indices $i$ were allowed to come from an arbitrary set. We will occasionally use the latter framework without further comment.

The following example will be crucially used throughout the rest of the article.

Example 4.3. Let $\Gamma$ be a finitely generated discrete group endowed with a proper length function $l$. Then the collection $B_{n}^{l}:=\operatorname{span}\left\{\lambda_{g} \mid l(g)=n\right\}, n \geq 0$, forms a filtration for the pair $\left(\mathrm{C}_{\mathrm{r}}^{*}(\Gamma), \tau_{\Gamma}\right)$ where $\tau_{\Gamma}$ is the canonical trace on $\mathrm{C}_{\mathrm{r}}^{*}(\Gamma)$.

\subsection{The quantum symmetry group of an orthogonal filtration.}

Theorem $4.4[8,18]$. Let $\left\{A_{i}\right\}_{i \geq 0}$ be an orthogonal filtration for a pair $\left(A, \tau_{A}\right)$ as above. Let $\mathcal{C}(\widetilde{A})$ be the category with objects as pairs $(G, \alpha)$ where $G$ is a compact quantum group, $\alpha$ is an action of $G$ on $A$ such that $\alpha\left(A_{i}\right) \subseteq A_{i} \otimes_{\text {alg }} \mathrm{C}(G)$ for each $i \geq 0$, and the morphisms being CQG morphisms intertwining the respective actions. Then there exists a universal initial object in the category $\mathcal{C}(\widetilde{A})$, called the quantum symmetry group of the filtration $\widetilde{A}$ and denoted by $\mathrm{QISO}(\widetilde{A})$. Moreover, the action of $\mathrm{QISO}(\widetilde{A})$ on $A$ is faithful (see Sect. 3.2 for the definition of faithfulness). 
Remark 4.5. As mentioned in Remark 4.2, the definition of an orthogonal filtration in [8] included an additional condition, namely that $\operatorname{Span}\left\{A_{i}: i \geq 0\right\}$ is a $*$-algebra. However, Thibault de Chanvalon showed [18] that the existence of the quantum symmetry group of an orthogonal filtration can be proved without assuming this extra condition.

Note that we assume throughout that the actions in our category are defined on the reduced level; in fact the construction of the quantum symmetry group in [8] gives naturally an action on the universal level (which then induces the reduced action). A certain care needs then to be taken when one interprets the intertwining relation with respect to the CQG morphisms (acting on the universal level), but this can be always dealt with, for example by exploiting the purely algebraic picture of the actions (see Lemma 4.8).

Remark 4.6. Given a pair $(G, \alpha) \in \mathcal{C}(\widetilde{A})$ we automatically deduce that the coaction $\alpha \in \operatorname{Mor}(A, A \otimes \mathrm{C}(G))$ is injective. This is because $\alpha$ preserves the faithful state $\tau_{A}$ as observed in [8], hence it is injective by Lemma 2.7 .

Remark 4.7. For a finitely generated countable group $\Gamma$ and a fixed word-length function $l$, consider the orthogonal filtration $\widetilde{B}:=\left(\mathrm{C}_{\mathrm{r}}^{*}(\Gamma), \tau_{\Gamma},\left\{B_{n}^{l}\right\}_{n \geq 0}\right)$ of Example 4.3. Then it can be easily seen that $(\hat{\Gamma}, \hat{\Delta})$ is an object of the category $\mathcal{C}(\widetilde{B})$; in particular we have a morphism from $\widehat{\Gamma}$ to $\operatorname{QISO}(\widetilde{B})$, represented by a Hopf *-homomorphism $\pi_{\Gamma} \in$ $\operatorname{Mor}\left(\mathrm{C}^{\mathrm{u}}(\mathrm{QISO}(\widetilde{B})), \mathrm{C}^{*}(\Gamma)\right)$. Moreover, in [12], it was proved that for $\Gamma=\mathbb{Z}_{n}(n \in$ $\mathbb{N}, n \neq 4) \mathrm{C}(\mathrm{QISO}(\widetilde{C})) \cong \mathrm{C}^{*}(\Gamma) \oplus \mathrm{C}^{*}(\Gamma)$.

As an immediate application of Theorem 4.4, one can make the following observations.

Lemma 4.8. Let $\widetilde{A}$ be as above and let $(G, \alpha)$ be an object in the category $\mathcal{C}(\widetilde{A})$. Then we have the following:

(1) if $\left\{a_{i j} \mid j=1,2, \ldots, \operatorname{dim}\left(A_{i}\right)\right\}$ is a basis of $A_{i}$, then there exist elements $q_{k j}^{i} \in$ $\operatorname{Pol}(G)\left(i \geq 0, k, j=1, \ldots, \operatorname{dim}\left(A_{i}\right)\right)$ such that

$$
\alpha\left(a_{i j}\right)=\sum_{k} a_{i k} \otimes q_{k j}^{i} \quad \text { for all } j, k=1,2, \ldots, \operatorname{dim}\left(A_{i}\right) .
$$

(2) The action $\alpha$ is faithful if and only if the $\mathrm{C}^{*}$-algebra generated by $\left\{q_{k j}^{i} \mid i \geq 0, j, k=\right.$ $\left.1,2, \ldots, \operatorname{dim}\left(A_{i}\right)\right\}$ is equal to $\mathrm{C}(G)$.

(3) If $\alpha$ is a faithful action, then the canonical morphism $($ in $\mathcal{C}(\widetilde{A}))$ from $\mathrm{C}^{\mathrm{u}}(\mathrm{QISO}(\widetilde{A}))$ to $\mathrm{C}(G)$ is surjective.

4.2. Orthogonal filtration of a twisted tensor product. Throughout this subsection we will work with the following notation: $\widetilde{A}:=\left(A, \tau_{A},\left\{A_{i}\right\}_{i \geq 0}\right)$ and $\widetilde{B}:=\left(B, \tau_{B},\left\{B_{j}\right\}_{j \geq 0}\right)$ will denote orthogonal filtrations of unital $C^{*}$-algebras $A$ and $B, \gamma_{A}$ and $\gamma_{B}$ will denote the canonical actions of $\operatorname{QISO}(\widetilde{A})$ on $A$ and $\operatorname{QISO}(\widetilde{B})$ on $B$, respectively, while $\mathrm{V} \in \mathcal{U}\left(\mathrm{C}_{0}(\widehat{\mathrm{QISO}(\widetilde{A})}) \otimes \mathrm{C}_{0}(\widehat{\mathrm{QISO}(\widetilde{B}})\right)$ will be a fixed bicharacter.

Let $(\alpha, \beta)$ be a V-Heisenberg pair on $\mathcal{H}$. We will work with a realization of $A \nabla_{\mathrm{V}} B$ inside $A \otimes B \otimes \mathbb{B}(\mathcal{H})$ defined via embeddings $j_{A}$ and $j_{B}$ described by (2.19).

Since $\gamma_{A}$ preserves $\tau_{A}$ and $\gamma_{B}$ preserves $\tau_{B}$, we can apply [35, Lemma 5.5] for completely positive maps to define a functional $\tau_{A} \otimes_{\mathrm{V}} \tau_{B}: A \otimes_{\mathrm{V}} B \rightarrow \mathbb{C}$ by

$$
\tau_{A} \bigotimes_{\mathrm{V}} \tau_{B}\left(j_{A}(a) j_{B}(b)\right)=\tau_{A}(a) \tau_{B}(b) \quad \text { for all } a \in A, b \in B .
$$


Proposition 4.9. The functional $\tau_{A} \bigotimes_{\mathrm{V}} \tau_{B}$ is a faithful state on $A \bigotimes_{\mathrm{V}} B$ and the triple $\widetilde{A} \bigotimes_{\mathrm{V}}$ $\widetilde{B}:=\left(A \bigotimes_{\mathrm{V}} B, \tau_{A} \bigotimes_{\mathrm{V}} \tau_{B},\left\{j_{A}\left(A_{i}\right) j_{B}\left(B_{j}\right)\right\}_{i, j \geq 0}\right)$ is an orthogonal filtration of $A \bigotimes_{\mathrm{V}} B$.

Proof. Define $\tau^{\prime}: A \otimes B \otimes \mathbb{B}(\mathcal{H}) \rightarrow \mathbb{B}(\mathcal{H})$ by $\tau^{\prime}:=\tau_{A} \otimes \tau_{B} \otimes \mathrm{id}_{\mathcal{H}}$. Then $\tau^{\prime}$ is unital and positive because $\tau_{A}$ and $\tau_{B}$ are states. By [35, Lemma 5.5], $\tau_{A} \bigotimes_{\mathrm{V}} \tau_{B}(\cdot) 1_{H}:=\left.\tau^{\prime}\right|_{A \bigotimes B}$. Since $\tau^{\prime}$ is faithful, so is its restriction $\tau_{A} \otimes_{\mathrm{V}} \tau_{B}$. Therefore, $\tau_{A} \otimes_{\mathrm{V}} \tau_{B}$ is a faithful state on $A \otimes_{\mathrm{V}} B$.

Let $S:=\operatorname{Span}\left\{j_{A}\left(A_{i}\right) j_{B}\left(B_{j}\right): i, j \geq 0\right\}$. Since the density of $S$ in $A \nabla_{\mathrm{V}} B$ is clear by the definition of $A \bigotimes_{\mathrm{V}} B$, we only need to prove that $\left\{j_{A}\left(A_{i}\right) j_{B}\left(B_{j}\right\}_{i, j \geq 0}\right.$ is orthogonal with respect to $\tau_{A} \bigotimes_{\mathrm{V}} \tau_{B}$. Indeed, for all $a_{i} \in A_{i}, b_{j} \in B_{j}, a_{k} \in A_{k}$ and $b_{l} \in B_{l}$, we have:

$$
\begin{aligned}
c:= & \tau_{A} \bigotimes_{\mathrm{V}} \tau_{B}\left(\left(j_{A}\left(a_{i}\right) j_{B}\left(b_{j}\right)\right)^{*} j_{A}\left(a_{k}\right) j_{B}\left(b_{l}\right)\right) \\
= & \tau_{A} \bigotimes_{\mathrm{V}} \tau_{B}\left(j_{B}\left(b_{j}^{*}\right) j_{A}\left(a_{i}^{*} a_{k}\right) j_{B}\left(b_{l}\right)\right) \\
= & \left(\tau_{A} \otimes \tau_{B} \otimes \operatorname{id}_{\mathcal{H}}\right)\left(\left(\left(\operatorname{id}_{B} \otimes \beta\right)\left(\gamma_{B}\left(b_{j}^{*}\right)\right)\right)_{23}\left(\left(\operatorname{id}_{A} \otimes \alpha\right)\left(\gamma_{A}\left(a_{i}^{*} a_{k}\right)\right)\right)_{13}\right. \\
& \left.\left(\left(\operatorname{id}_{B} \otimes \beta\right)\left(\gamma_{B}\left(b_{l}\right)\right)\right)_{23}\right) \\
= & \left.\left(\tau_{B} \otimes \operatorname{id}_{\mathcal{H}}\right)\left(\left(\operatorname{id}_{B} \otimes \beta\right)\left(\gamma_{B}\left(b_{j}^{*}\right)\right)\right)\left(\left(\tau_{A} \otimes \alpha\right)\left(\gamma_{A}\left(a_{i}^{*} a_{k}\right)\right)\right)\left(\left(\operatorname{id}_{B} \otimes \beta\right)\left(\gamma_{B}\left(b_{l}\right)\right)\right)\right) \\
= & \tau_{A}\left(a_{i}^{*} a_{k}\right)\left(\tau_{B} \otimes \beta\right)\left(\gamma_{B}\left(b_{j}^{*} b_{l}\right)\right)=\tau_{A}\left(a_{i}^{*} a_{k}\right) \tau_{B}\left(b_{j}^{*} b_{l}\right) 1_{\mathcal{B}(\mathcal{H}) .}
\end{aligned}
$$

Therefore, if $(i, j) \neq(k, l)$, then $c=0$ since $\tau_{A}\left(a_{i}^{*} a_{k}\right)=0$ if $i \neq k$ and $\tau_{B}\left(b_{j}^{*} b_{l}\right)=0$ if $j \neq l$. This proves that $\left\{j_{A}\left(A_{i}\right) j_{B}\left(B_{j}\right)\right\}_{i, j \geq 0}$ yields an orthogonal filtration with respect to $\tau_{A} \otimes \tau_{B}$.

Let then $\left(G, \gamma_{A}^{\prime}\right)$ and $\left(H, \gamma_{B}^{\prime}\right)$ be objects in $\mathcal{C}(\widetilde{A})$ and $\mathcal{C}(\widetilde{B})$, respectively. Suppose $\mathrm{V}_{\mathcal{L}} \in \mathcal{U}\left(\mathrm{C}_{0}(\hat{G}) \times \mathrm{C}_{0}(\hat{H})\right)$ is a bicharacter. Then universality of $\operatorname{QISO}(\tilde{A})$ and $\operatorname{QISO}(\widetilde{B})$ gives the existence Hopf ${ }^{*}$-homomorphisms $f_{1}: \mathrm{C}^{\mathrm{u}}(\mathrm{QISO}(\widetilde{A})) \rightarrow \mathrm{C}(G)$ and $f_{2}: \mathrm{C}^{\mathrm{u}}(\mathrm{QISO}(\widetilde{B})) \rightarrow \mathrm{C}(H)$ such that $\left(\mathrm{id}_{A} \otimes f_{1}\right) \circ \gamma_{A}^{\mathrm{u}}=\gamma_{A}^{\prime}$ and $\left(\operatorname{id}_{B} \otimes f_{2}\right) \circ \gamma_{B}^{\mathrm{u}}=\gamma_{B}^{\prime}$. These admit universal lifts and by Theorem 2.10 induce dual Hopf *-homomorphisms

$$
\left.\left.\hat{f_{1}}: \mathrm{C}_{0}(\hat{G}) \rightarrow \mathrm{C}_{0}(\widehat{\mathrm{QISO}(\widetilde{A}})\right) \quad \text { and } \quad \hat{f}_{2}: \mathrm{C}_{0}(\hat{H}) \rightarrow \mathrm{C}_{0}(\widehat{\mathrm{QISO}(\widetilde{B}})\right) .
$$

The latter maps allow us to define a bicharacter $\mathrm{V} \in \mathcal{U}\left(\mathrm{C}_{0}(\widehat{\mathrm{QISO}(\widetilde{A})}) \otimes \mathrm{C}_{0}(\widehat{\mathrm{QISO}(\widetilde{B}})\right)$ by the formula $\mathrm{V}=\left(\hat{f}_{1} \otimes \hat{f}_{2}\right)\left(\mathrm{V}_{1}\right)$.

Corollary 4.10. In the situation above, there is a faithful state $\tau_{A} \bigotimes_{\mathrm{V}_{1}} \tau_{B}$ on $A \bigotimes_{\mathrm{V}_{1}} B$ such that the triplet $\left(A \nabla_{\mathrm{V}_{1}} B, \tau_{A} \bigotimes_{\mathrm{V}_{1}} \tau_{B},\left\{j_{A}^{\prime}\left(A_{i}\right) j_{B}^{\prime}\left(B_{j}\right)\right\}_{i, j \geq 0}\right)$ is an orthogonal filtration of $A \otimes_{\mathrm{V}_{1}} B$, where $j_{A}^{\prime}$ and $j_{B}^{\prime}$ are embeddings of $A$ and $B$ into $A \nabla_{\mathrm{V}_{1}} B$.

Proof. Clearly, $\gamma_{A}^{\prime}$ and $\gamma_{B}^{\prime}$ are injective coactions because they preserve $\tau_{A}$ and $\tau_{B}$ respectively. Now Lemma 2.11 shows that $\gamma_{A}^{\prime}$ and $\gamma_{B}^{\prime}$ are induced by the Hopf*-homomorphism

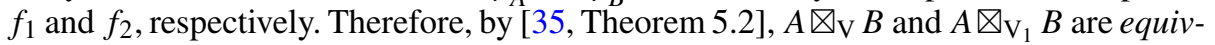
alent: there is an isomorphism $\Theta \in \operatorname{Mor}\left(A \bigotimes_{\mathrm{V}} B, A \bigotimes_{\mathrm{V}_{1}} B\right)$ such that

$$
\Theta \circ j_{A}=j_{A}^{\prime}, \quad \Theta \circ j_{B}=j_{B}^{\prime} .
$$

Then $\tau_{A} \bigotimes_{\mathrm{V}_{1}} \tau_{B}:=\left(\tau_{A} \bigotimes_{\mathrm{V}} \tau_{B}\right) \circ \Theta^{-1}$ defines a faithful state on $A \bigotimes_{\mathrm{V}_{1}} B$ and the double-indexed family $\left\{j_{A}^{\prime}\left(A_{i}\right) j_{B}^{\prime}\left(B_{j}\right)\right\}_{i, j \geq 0}$ defines an orthogonal filtration of $A \bigotimes_{\mathrm{V}_{1}} B$ with respect to $\tau_{A} \otimes_{\mathrm{V}_{1}} \tau_{B}$. 


\section{Quantum Symmetries of Twisted Tensor Product}

Let $\widetilde{A}:=\left(A, \tau_{A},\left\{A_{i}\right\}_{i \geq 0}\right)$ and $\widetilde{B}:=\left(B, \tau_{B},\left\{B_{j}\right\}_{j \geq 0}\right)$ be orthogonal filtrations of unital $C^{*}$-algebras $A$ and $B$. Let $\gamma_{A}$ and $\gamma_{B}$ denote the actions of $\operatorname{QISO}(\widetilde{A})$ and $\operatorname{QISO}(\widetilde{B})$ on $A$ and $B$, respectively. Let $\left(G, \gamma_{A}^{\prime}\right)$ and $\left(H, \gamma_{B}^{\prime}\right)$ be objects in $\mathcal{C}(\widetilde{A})$ and $\mathcal{C}(\widetilde{B})$ respectively, and suppose we have a bicharacter $\mathrm{V}_{1} \in \mathcal{U}\left(\mathrm{C}_{0}(\hat{G}) \otimes \mathrm{C}_{0}(\hat{H})\right)$. Let $\mathrm{V} \in$ $\left.\mathcal{U}\left(\mathrm{C}_{0}(\widehat{\mathrm{QISO}(\widetilde{A}})\right) \otimes \mathrm{C}_{0}(\widehat{\mathrm{QISO}(\widetilde{B}})\right)$ be the associated bicharacter as in Corollary 4.10. By Proposition 4.9, $\left\{j_{A}\left(A_{i}\right) j_{B}\left(B_{j}\right)\right\}_{i, j \geq 0}$ is an orthogonal filtration of $A \bigotimes_{\mathrm{V}} B$ with respect to the state $\tau_{A} \nabla_{\mathrm{V}} \tau_{B}$. The resulting triple will be denoted by $\widetilde{A} \nabla_{\mathrm{V}} \widetilde{B}$. Finally, let $\mathfrak{D}_{\mathrm{V}}$ denote the Drinfeld double of $\operatorname{QISO}(\widetilde{A})$ and $\operatorname{QISO}(\widetilde{B})$ with respect to the bicharacter V. The aim of this section is to prove Theorem 5.4 which states that $\mathrm{QISO}\left(\widetilde{A} \nabla_{\mathrm{V}_{1}} \widetilde{B}\right)$ is isomorphic to $\mathfrak{D}_{\mathrm{V}}$. It turns out that this conclusion can be easily derived from the following theorem:

Theorem 5.1. Let $\widetilde{A}, \widetilde{B}$ be orthogonal filtrations, and fix a reduced bicharacter $\mathrm{V} \in$ $\left.\left.\mathcal{U}\left(\mathrm{C}_{0}(\widehat{\mathrm{QISO}(\widetilde{A}})\right) \otimes \mathrm{C}_{0}(\widehat{\mathrm{QISO}(\widetilde{B}})\right)\right)$. The quantum symmetry group $\mathrm{QISO}\left(\widetilde{A} \otimes_{\mathrm{V}} \widetilde{B}\right)$, whose existence is guaranteed by Proposition 4.9, is isomorphic to $\mathfrak{D}_{\mathrm{V}}$, the Drinfeld double of $\mathrm{QISO}(\widetilde{A})$ and $\mathrm{QISO}(\widetilde{B})$ with respect to the bicharacter $\mathrm{V}$.

For the rest of the section, the symbol $\mathrm{V}$ will denote a fixed reduced bicharacter belonging to $\left.\left.\mathcal{U}\left(\mathrm{C}_{0}(\widehat{\mathrm{QSO}(\widetilde{A}})\right) \otimes \mathrm{C}_{0}(\widehat{\mathrm{QISO}(\widetilde{B}})\right)\right)$. As a preparation for proving Theorem 5.1, we will first prove some auxiliary results. Remark 4.6 shows that the coactions $\gamma_{A}$ and $\gamma_{B}$ are injective. By the same argument the actions $\gamma_{A}^{\mathrm{u}}$ and $\gamma_{B}^{\mathrm{u}}$ defined on the universal level are normal. Thus, by Lemma 3.4, there is a coaction $\gamma_{A}^{\mathrm{u}} \bowtie_{\mathrm{V}} \gamma_{B}^{\mathrm{u}}$ of $\mathrm{C}_{0}^{\mathrm{u}}\left(\mathfrak{D}_{\mathrm{V}}\right)$ on $A \nabla_{\mathrm{V}} B$ satisfying (3.4). This allows us to show the following fact.

Lemma 5.2. The pair $\left(\mathfrak{D}_{\mathrm{V}}, \gamma_{A}^{\mathrm{u}} \bowtie_{\mathrm{V}} \gamma_{B}^{\mathrm{u}}\right)$ is an object in the category $\mathcal{C}\left(\widetilde{A} \nabla_{\mathrm{V}} \widetilde{B}\right)$. Moreover, $\gamma_{A}^{\mathrm{u}} \bowtie_{\mathrm{V}} \gamma_{B}^{\mathrm{u}}$ is a faithful coaction.

Proof. Let $i \geq 0$, and let $\left\{v_{i, x} \mid x=1,2, \ldots, \operatorname{dim}\left(A_{i}\right)\right\}$ be a basis of $A_{i}$. By Lemma 4.8, we have elements $\left\{q_{k l}^{i} \mid k, l=1,2, \ldots, \operatorname{dim}\left(A_{i}\right)\right\} \in \operatorname{Pol}(\mathrm{QISO}(\widetilde{A})) \subset \mathrm{C}^{\mathrm{u}}(\mathrm{QISO}(\widetilde{A}))$ such that for each $k=1, \ldots, \operatorname{dim}\left(A_{i}\right)$

$$
\gamma_{A}^{\mathrm{u}}\left(v_{i, k}\right)=\sum_{l=1}^{\operatorname{dim}\left(A_{i}\right)} v_{i, l} \otimes q_{l k}^{i} .
$$

Moreover, by virtue of (3.4),

$$
\gamma_{A}^{\mathrm{u}} \bowtie \mathrm{V} \gamma_{B}^{\mathrm{u}}\left(j_{A}\left(v_{i, k}\right)\right)=\sum_{l=1}^{\operatorname{dim}\left(A_{i}\right)} j_{A}\left(v_{i, l}\right) \otimes \rho^{\mathrm{u}}\left(q_{l k}^{i}\right) .
$$

Similarly, if $j \geq 0$ and $\left\{w_{j, m} \mid m=1,2, \ldots, \operatorname{dim}\left(B_{j}\right)\right\}$ is a basis of $B_{j}$, then we have elements $\left\{r_{m n}^{j} \mid m, n=1,2, \ldots, \operatorname{dim}\left(B_{j}\right)\right\} \in \mathrm{C}^{\mathrm{u}}(\mathrm{QISO}(\widetilde{B}))$ such that for $m=$ $1,2, \ldots, \operatorname{dim}\left(B_{j}\right)$

$$
\gamma_{A}^{\mathrm{u}} \bowtie \mathrm{V} \gamma_{B}^{\mathrm{u}}\left(j_{B}\left(w_{j, m}\right)\right)=\sum_{n=1}^{\operatorname{dim}\left(B_{j}\right)} j_{B}\left(w_{j, n}\right) \otimes \theta^{\mathrm{u}}\left(r_{n m}^{j}\right) .
$$


Therefore, we can conclude that

$$
\gamma_{A}^{\mathrm{u}} \bowtie_{\mathrm{V}} \gamma_{B}^{\mathrm{u}}\left(j_{A}\left(v_{i, k}\right) j_{B}\left(w_{j, m}\right)\right)=\sum_{l, n} j_{A}\left(v_{i, l}\right) j_{B}\left(w_{j, n}\right) \otimes \rho^{\mathrm{u}}\left(q_{l k}^{i}\right) \theta^{\mathrm{u}}\left(r_{n m}^{j}\right) .
$$

Thus, the map $\gamma_{A}^{\mathrm{u}} \bowtie \mathrm{V} \gamma_{B}^{\mathrm{u}}$ preserves the subspace $j_{A}\left(A_{i}\right) j_{B}\left(B_{j}\right)$ for each $i, j \geq 0$. This proves the first assertion.

Now we prove that the action $\gamma_{A}^{\mathrm{u}} \bowtie \mathrm{V} \gamma_{B}^{\mathrm{u}}$ is faithful. By Lemma 4.8 (2) and by the equality (5.1) it is enough to show that the $C^{*}$-algebra generated by the set $\left\{\rho^{\mathrm{u}}\left(q_{l, k}^{i}\right)\right.$ $\left.\theta^{\mathrm{u}}\left(r_{n, m}^{j}\right) \mid i, j \geq 0, l, k=1, \ldots, \operatorname{dim}\left(A_{i}\right), n, m=1, \ldots, \operatorname{dim}\left(B_{j}\right)\right\}$ is equal to $\mathrm{C}^{\mathrm{u}}\left(\mathfrak{D}_{\mathrm{V}}\right)$.

Since $\gamma_{A}^{\mathrm{u}}$ and $\gamma_{B}^{\mathrm{u}}$ are faithful coactions, we have:

$$
\begin{aligned}
& C^{*}\left\{q_{l k}^{i} \mid i \geq 0, l, k=1, \ldots, \operatorname{dim}\left(A_{i}\right)\right\}=\mathrm{C}^{\mathrm{u}}(\operatorname{QISO}(\widetilde{A})), \\
& C^{*}\left\{r_{n m}^{j} \mid j \geq 0, n, m=1, \ldots, \operatorname{dim}\left(B_{j}\right)\right\}=\mathrm{C}^{\mathrm{u}}(\mathrm{QISO}(\widetilde{B})) .
\end{aligned}
$$

$\operatorname{As} \mathrm{C}^{\mathrm{u}}\left(\mathfrak{D}_{\mathrm{V}}\right)=\rho^{\mathrm{u}}\left(\mathrm{C}^{\mathrm{u}}(\mathrm{QISO}(\widetilde{A}))\right) \theta^{\mathrm{u}}\left(\mathrm{C}^{\mathrm{u}}(\mathrm{QISO}(\widetilde{B}))\right)$, the proof is completed.

Next we prove the following auxiliary result.

Lemma 5.3. Let $\gamma^{\mathrm{u}}$ denote the coaction of $\mathrm{C}^{\mathrm{u}}\left(\mathrm{Q} \operatorname{ISO}\left(\widetilde{A} \otimes_{\mathrm{V}} \widetilde{B}\right)\right)$ on $A \bigotimes_{\mathrm{V}} B$. Then

$$
\begin{aligned}
& \left(\tau_{A} \bigotimes_{\mathrm{V}} \operatorname{id}_{B} \otimes \operatorname{id}_{\operatorname{QISO}\left(\widetilde{A} \bigotimes_{\mathrm{V}} \widetilde{B}\right)}\right)\left(\gamma^{\mathrm{u}}\left(j_{A}(a) j_{B}(b)\right)\right)=\tau_{A}(a) \gamma^{\mathrm{u}}\left(j_{B}(b)\right), \\
& \left(\operatorname{id}_{A} \bigotimes_{\mathrm{V}} \tau_{B} \otimes \operatorname{id}_{\mathrm{QISO}\left(\widetilde{A} \bigotimes_{\mathrm{V}} \widetilde{B}\right)}\right)\left(\gamma^{\mathrm{u}}\left(j_{A}(a) j_{B}(b)\right)\right)=\tau_{B}(b) \gamma^{\mathrm{u}}\left(j_{A}(a)\right)
\end{aligned}
$$

for all $a \in A$ and $b \in B$.

Proof. By [35, Lemma 5.5], concerning equivariant completely positive maps, we have

$$
\tau_{A} \bigotimes_{\mathrm{V}} \operatorname{id}_{B}\left(j_{A}(a) j_{B}(b)\right)=\tau_{A}(a) j_{B}(b), \quad \operatorname{id}_{A} \nabla_{\mathrm{V}} \tau_{B}\left(\left(j_{A}(a) j_{B}(b)\right)=\tau_{B}(b) j_{A}(a)\right.
$$

for all $a \in A, b \in B$.

By Lemmas 5.2 and 4.8 (3) it follows that there is a unique surjective Hopf*-homomorphism $q: \mathrm{C}^{\mathrm{u}}\left(\mathrm{QISO}\left(\widetilde{A} \otimes_{\mathrm{V}} \widetilde{B}\right)\right) \rightarrow \mathrm{C}^{\mathrm{u}}\left(\mathfrak{D}_{\mathrm{V}}\right)$ such that

$$
\begin{aligned}
& \left(\mathrm{id}_{A \bigotimes_{\mathrm{V}} B} \otimes q\right) \circ \gamma^{\mathrm{u}} \circ j_{A}=\gamma_{A}^{\mathrm{u}} \bowtie \gamma_{B}^{\mathrm{u}} \circ j_{A}=\left(j_{A} \otimes \rho^{\mathrm{u}}\right) \circ \gamma_{A}^{\mathrm{u}}, \\
& \left(\mathrm{id}_{A \bigotimes_{\mathrm{V}} B} \otimes q\right) \circ \gamma^{\mathrm{u}} \circ j_{B}=\gamma_{A}^{\mathrm{u}} \bowtie \gamma_{B}^{\mathrm{u}} \circ j_{B}=\left(j_{B} \otimes \theta^{\mathrm{u}}\right) \circ \gamma_{B}^{\mathrm{u}} .
\end{aligned}
$$

Notice that the morphism $\gamma_{A}^{\mathrm{u}} \bowtie_{\mathrm{V}} \gamma_{B}^{\mathrm{u}}$ is injective as it preserves the faithful state $\tau_{A} \otimes_{\mathrm{V}} \tau_{B}$. Using (5.4), (5.5) and (5.6) we get

$$
\begin{aligned}
& \left(\tau_{A} \otimes_{\mathrm{V}} \operatorname{id}_{B} \otimes q\right)\left(\gamma^{\mathrm{u}}\left(j_{A}(a) j_{B}(b)\right)\right) \\
& \quad=\left(\tau_{A} \otimes_{\mathrm{V}} \operatorname{id}_{B} \otimes \mathrm{id}_{\mathrm{QISO}\left(\tilde{A} \otimes_{\mathrm{V}} \widetilde{B}\right)\left(\left(j_{A} \otimes \rho^{\mathrm{u}}\right)\left(\gamma_{A}^{\mathrm{u}}(a)\right)\left(j_{B} \otimes \theta^{\mathrm{u}}\right)\left(\gamma_{B}^{\mathrm{u}}(b)\right)\right)}\right. \\
& \quad=\left(\tau_{A} \otimes \rho^{\mathrm{u}}\right)\left(\gamma_{A}^{\mathrm{u}}(a)\right)\left(j_{B} \otimes \theta^{\mathrm{u}}\right)\left(\gamma_{B}^{\mathrm{u}}(b)\right) \\
& \quad=\tau_{A}(a)\left(j_{B} \otimes \theta^{\mathrm{u}}\right)\left(\gamma_{B}^{\mathrm{u}}(b)\right)=\left(\operatorname{id}_{A \bigotimes_{\mathrm{V}} B} \otimes q\right)\left(\tau_{A}(a) \gamma^{\mathrm{u}}\left(j_{B}(b)\right)\right) .
\end{aligned}
$$

Note that we have shown above that

$$
\begin{aligned}
& \left(\operatorname{id}_{A \bigotimes_{\mathrm{V} B}} \otimes q\right)\left(\left(\tau_{A} \bigotimes_{\mathrm{V}} \mathrm{id}_{B} \otimes \mathrm{id}\right)\left(\gamma^{\mathrm{u}}\left(j_{A}(a) j_{B}(b)\right)\right)\right) \\
& \quad=\left(\operatorname{id}_{A \bigotimes_{\mathrm{V}} B} \otimes q\right)\left(\tau_{A}(a) \gamma^{\mathrm{u}}\left(j_{B}(b)\right)\right) .
\end{aligned}
$$


The equation $(\mathrm{id} \otimes q) \circ \gamma^{\mathrm{u}}=\gamma_{A}^{\mathrm{u}} \bowtie \gamma_{B}^{\mathrm{u}}$ implies that $\mathrm{id} \otimes q$ is injective on $\operatorname{Ran}\left(\gamma^{\mathrm{u}}\right)$. Thus it follows that (5.2) holds, if only we can show that $\left(\tau_{A} \nabla_{\mathrm{V}} \mathrm{id}_{B} \otimes \mathrm{id}\right)\left(\gamma^{\mathrm{u}}\left(j_{A}(a) j_{B}(b)\right)\right)$ belongs to the image of $\gamma^{\mathrm{u}}$. For that (by density) we may assume that $a \in A_{i}, b \in$ $B_{j}$ for some $i, j \geq 0$. Since by definition the coaction $\gamma^{\mathrm{u}}$ preserves the subspaces $j_{A}\left(A_{i}\right) j_{B}\left(B_{j}\right)$ for all $i, j \geq 0$, it has to preserve the subspaces $j_{A}\left(A_{i}\right)$ and $j_{B}\left(B_{j}\right)$ and hence $\gamma^{\mathrm{u}}\left(j_{A}(a)\right)=\sum_{k=1}^{n} j_{A}\left(a_{k}\right) \otimes x_{k}, \gamma^{\mathrm{u}}\left(j_{B}(b)\right)=\sum_{k=1}^{n} j_{B}\left(b_{k}\right) \otimes y_{k}$ for some $n \in \mathbb{N}$, $a_{1}, \ldots, a_{n} \in A_{i}, b_{1}, \ldots, b_{n} \in B_{j}$ and $x_{1}, \ldots, x_{n}, y_{1}, \ldots, y_{n} \in \mathrm{C}^{\mathrm{u}}\left(\mathrm{QISO}\left(\widetilde{A} \nabla_{\mathrm{V}} \widetilde{B}\right)\right)$. Thus

$$
\begin{aligned}
\left(\tau_{A}\right. & \left.\otimes_{\mathrm{V}} \operatorname{id}_{B} \otimes \mathrm{id}\right)\left(\gamma^{\mathrm{u}}\left(j_{A}(a) j_{B}(b)\right)\right) \\
& =\sum_{j, k=1}^{n}\left(\tau_{A} \otimes_{\mathrm{V}} \operatorname{id}_{B} \otimes \mathrm{id}\right)\left(j_{A}\left(a_{j}\right) j_{B}\left(b_{k}\right) \otimes x_{j} y_{k}\right) \\
& =\sum_{j, k=1}^{n} j_{A}\left(\tau_{A}\left(a_{j}\right)\right) j_{B}\left(b_{k}\right) \otimes x_{j} y_{k}=\left(\sum_{j=1}^{n} j_{A}\left(\tau_{A}\left(a_{j}\right)\right) j_{B}(1) \otimes x_{j}\right) \gamma^{\mathrm{u}}\left(j_{B}(b)\right) \\
& =\left(\tau_{A} \otimes_{\mathrm{V}} \tau_{B} \otimes \mathrm{id}\right)\left(\gamma^{\mathrm{u}}\left(j_{A}(a) j_{B}(1)\right)\right) \gamma^{\mathrm{u}}\left(j_{B}(b)\right) \\
& =\left(\tau_{A} \otimes_{\mathrm{V}} \tau_{B}\left(j_{A}(a) j_{B}(1)\right)\right) \gamma^{\mathrm{u}}\left(j_{B}(b)\right)=\tau_{A}(a) \gamma^{\mathrm{u}}\left(j_{B}(b)\right),
\end{aligned}
$$

where in the second equality we used [35, Lemma 5.5]. This shows the desired containment and completes the proof of (5.2).

Similarly, we can show that (5.3) holds.

We are now ready to prove Theorem 5.1.

Proof of Theorem 5.1. From the Podleś condition for $\gamma^{\mathrm{u}}$ we get

$$
\gamma^{\mathrm{u}}(A \otimes B)\left(1_{A \bigotimes_{\mathrm{V}} B} \otimes \mathrm{C}^{\mathrm{u}}\left(\mathrm{QISO}\left(\widetilde{A} \otimes_{\mathrm{V}} \widetilde{B}\right)\right)\right)=A \otimes B \otimes \mathrm{C}^{\mathrm{u}}\left(\mathrm{Q} \operatorname{ISO}\left(\widetilde{A} \otimes_{\mathrm{V}} \widetilde{B}\right)\right)
$$

Applying $\tau_{A} \bigotimes_{\mathrm{V}} \operatorname{id}_{B} \otimes \operatorname{id}_{\mathrm{QISO}\left(\widetilde{A} \bigotimes_{\mathrm{V}} \widetilde{B}\right)}$ to the both sides of the above equality and using (5.2) and (5.4) gives

$$
\gamma^{\mathrm{u}}\left(j_{B}(B)\right)\left(1_{j_{B}(B)} \otimes \mathrm{C}^{\mathrm{u}}\left(\mathrm{QISO}\left(\tilde{A} \nabla_{\mathrm{V}} \widetilde{B}\right)\right)\right)=j_{B}(B) \otimes \mathrm{C}^{\mathrm{u}}\left(\mathrm{QISO}\left(\widetilde{A} \nabla_{\mathrm{V}} \widetilde{B}\right)\right) .
$$

Thus, $\gamma^{\mathrm{u}}\left(j_{B}(B)\right) \subseteq j_{B}(B) \otimes \mathrm{C}^{\mathrm{u}}\left(\mathrm{QISO}\left(\tilde{A} \otimes_{\mathrm{V}} \widetilde{B}\right)\right)$. Therefore, $\tilde{\gamma}_{B}:=\left(j_{B}^{-1} \otimes\right.$ $\left.\operatorname{id}_{\mathrm{QISO}\left(\widetilde{A} \mathbb{\mathrm { V }}_{\mathrm{V}} \widetilde{B}\right)}\right) \circ \gamma^{\mathrm{u}} \circ j_{B}$ defines a coaction of $\mathrm{C}^{\mathrm{u}}\left(\mathrm{Q} \operatorname{ISO}\left(\widetilde{A} \bigotimes_{\mathrm{V}} \widetilde{B}\right)\right)$ on $B$. Moreover, $\left(\mathrm{QISO}\left(\widetilde{A} \nabla_{\mathrm{V}} \widetilde{B}\right), \tilde{\gamma}_{B}\right)$ is an object in $\mathcal{C}(\widetilde{B})$ and so by Theorem 4.4 , there is a Hopf*-homomorphism $\theta_{1}: \mathrm{C}^{\mathrm{u}}(\mathrm{Q} I S O(\widetilde{B})) \rightarrow \mathrm{C}^{\mathrm{u}}\left(\mathrm{QISO}\left(\widetilde{A} \nabla_{\mathrm{V}} \widetilde{B}\right)\right)$ such that $\left(\operatorname{id}_{B} \otimes \theta_{1}\right) \circ \gamma_{B}^{\mathrm{u}}=\tilde{\gamma}_{B}$. This yields the following equality:

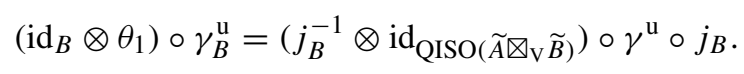

Hence,

$$
\left(j_{B} \otimes \theta_{1}\right) \circ \gamma_{B}^{\mathrm{u}}=\gamma^{\mathrm{u}} \circ j_{B} .
$$

Similarly, we can show that there is a coaction $\tilde{\gamma}_{A}$ of $\mathrm{C}^{\mathrm{u}}\left(\underset{\mathcal{A}}{(\mathcal{A} O}\left(\widetilde{A} \otimes_{\mathrm{V}} \widetilde{B}\right)\right)$ on $A$ and a Hopf *-homomorphism $\rho_{1}: \mathrm{C}^{\mathrm{u}}(\mathrm{QISO}(\widetilde{A})) \rightarrow \mathrm{C}^{\mathrm{u}}\left(\mathrm{QISO}\left(\widetilde{A} \otimes_{\mathrm{V}} \widetilde{B}\right)\right)$ such that

$$
\left(j_{A} \otimes \rho_{1}\right) \circ \gamma_{A}^{\mathrm{u}}=\gamma^{\mathrm{u}} \circ j_{A} .
$$


By the universal property of $\mathrm{C}^{\mathrm{u}}\left(\mathfrak{D}_{\mathrm{V}}\right)$ proved in Theorem 3.5 , there is a unique Hopf ${ }^{*}$-homomorphism $\Psi: \mathrm{C}^{\mathrm{u}}\left(\mathfrak{D}_{\mathrm{V}}\right) \rightarrow \mathrm{C}^{\mathrm{u}}\left(\mathrm{QISO}\left(\widetilde{A} \otimes_{\mathrm{V}} \widetilde{B}\right)\right)$ such that $\Psi \circ \rho^{\mathrm{u}}=\rho_{1}, \Psi \circ \theta^{\mathrm{u}}=$ $\theta_{1}$ and

$$
\begin{aligned}
& \left(\operatorname{id}_{A \bigotimes_{\mathrm{V}} B} \otimes \Psi\right) \circ \gamma_{A}^{\mathrm{u}} \bowtie \gamma_{B}^{\mathrm{u}} \circ j_{A}=\gamma^{\mathrm{u}} \circ j_{A} \\
& \left(\operatorname{id}_{A \bigotimes_{\mathrm{V}} B} \otimes \Psi\right) \circ \gamma_{A}^{\mathrm{u}} \bowtie \gamma_{B}^{\mathrm{u}} \circ j_{B}=\gamma^{\mathrm{u}} \circ j_{B} .
\end{aligned}
$$

Using (5.8), (5.10) and (5.5) we have

$$
\left(\mathrm{id}_{A \bigotimes_{\mathrm{V} B}} \otimes \Psi \circ q\right) \circ \gamma^{\mathrm{u}} \circ j_{A}=\left(j_{A} \otimes \Psi \circ \rho^{\mathrm{u}}\right) \circ \gamma_{A}^{\mathrm{u}}=\left(j_{A} \otimes \rho_{1}\right) \circ \gamma_{A}^{\mathrm{u}}=\gamma^{\mathrm{u}} \circ j_{A}
$$

Similarly, we can show that $\left(\operatorname{id}_{A \bigotimes_{\mathrm{V} B}} \otimes \Psi \circ q\right) \circ \gamma^{\mathrm{u}} \circ j_{B}=\gamma^{\mathrm{u}} \circ j_{B}$. Therefore, for all $\omega \in\left(A \otimes_{\mathrm{V}} B\right)^{\prime}$ we have

$$
(\Psi \circ q)\left(\left(\omega \otimes \operatorname{id}_{\mathrm{QISO}\left(\widetilde{A} \otimes_{\mathrm{V}} \widetilde{B}\right)}\right)\left(\gamma^{\mathrm{u}}(x)\right)\right)=\left(\omega \otimes \operatorname{id}_{\mathrm{QISO}\left(\widetilde{A} \otimes_{\mathrm{V}} \widetilde{B}\right)}\right)\left(\gamma^{\mathrm{u}}(x)\right)
$$

for all $x \in A \bigotimes_{\mathrm{V}} B$. Faithfulness of $\gamma^{\mathrm{u}}$ gives $\Psi \circ q(c)=c$ for all $c \in \mathrm{C}^{\mathrm{u}}\left(\mathrm{QISO}\left(\widetilde{A} \otimes_{\mathrm{V}} \widetilde{B}\right)\right)$.

A similar computation gives

$$
\left(\operatorname{id}_{A \bigotimes_{\mathrm{V}} B} \otimes q \circ \Psi\right)\left(\gamma_{A}^{\mathrm{u}} \bowtie \gamma_{B}^{\mathrm{u}}\left(j_{A}(a) j_{B}(b)\right)\right)=\gamma_{A}^{\mathrm{u}} \bowtie \gamma_{B}^{\mathrm{u}}\left(j_{A}(a) j_{B}(b)\right)
$$

for all $a \in A, b \in B$. Finally, by talking slices with $\omega \in\left(A \nabla_{\mathrm{V}} B\right)^{\prime}$ on the first leg of the the both sides in the above equation and using faithfulness of $\gamma_{A}^{\mathrm{u}} \bowtie \gamma_{B}^{\mathrm{u}}$ we obtain $(q \circ \Psi)(d)=d$ for all $d \in \mathrm{C}^{\mathrm{u}}\left(\mathfrak{D}_{\mathrm{V}}\right)$.

Thus, we are in a position to prove the main result of this article:

Theorem 5.4. Let $\left(G, \gamma_{A}^{\prime}\right)$ and $\left(H, \gamma_{B}^{\prime}\right)$ be objects in $\mathcal{C}(\widetilde{A})$ and $\mathcal{C}(\widetilde{B})$ respectively, and suppose we have a bicharacter $\mathrm{V}_{1} \in \mathcal{U}\left(\mathrm{C}_{0}(\hat{G}) \otimes \mathrm{C}_{0}(\hat{H})\right)$. Let $\mathrm{V} \in \mathcal{U}\left(\mathrm{C}_{0}(\widehat{\mathrm{QISO}(\widetilde{A}})\right) \otimes$ $\mathrm{C}_{0}(\mathrm{QISO}(\widetilde{B}))$ be the associated bicharacter as in Corollary 4.10. Then the quantum symmetry group $\mathrm{QISO}\left(\widetilde{A} \otimes_{\mathrm{V}_{1}} \widetilde{B}\right)$ is isomorphic to $\mathfrak{D}_{\mathrm{V}}$.

Proof. The result is an immediate consequence of Theorem 5.1 and of Corollary 4.10 and its proof.

In particular, we can choose $\left.\mathrm{V}=1 \in \mathcal{U}\left(\mathrm{C}_{0}(\widehat{\mathrm{QISO}(\widetilde{A}})\right) \otimes \mathrm{C}_{0}(\widehat{\mathrm{QISO}(\widetilde{B}})\right)$. Then $A \nabla_{\mathrm{V}}$ $B \cong A \otimes B$. Also, by virtue of [39, Example 5.10] the reduced Drinfeld double of $\operatorname{QISO}(\widetilde{A})$ and $\mathrm{QISO}(\widetilde{B})$ with respect to $\mathrm{V}$ is $\mathrm{QISO}(\widetilde{A}) \otimes \operatorname{QISO}(\widetilde{B})$. Thus, denoting the filtration of $A \otimes B$ coming from Proposition 4.9 by $\widetilde{A} \otimes \widetilde{B}$ and using the standard Cartesian product construction for compact quantum groups (so that $\mathrm{C}^{\mathrm{u}}(G \times H)=$ $\left.\mathrm{C}^{\mathrm{u}}(G) \otimes_{\max } \mathrm{C}^{\mathrm{u}}(H)\right)$ we obtain the following corollary.

Corollary 5.5. The quantum symmetry group $\mathrm{QISO}(\widetilde{A} \otimes \widetilde{B})$ is isomorphic to $\mathrm{QISO}(\widetilde{A}) \times$ $\operatorname{QISO}(\widetilde{B})$.

In the next example, we apply Theorem 5.4 to describe the quantum symmetry group of a class of Rieffel deformations of unital $\mathrm{C}^{*}$-algebras by actions of compact groups $[25,38]$. These are examples which are not necessarily of the crossed product type; the next section is devoted to the examples arising as reduced crossed products. 
Example 5.6. Let $A$ and $B$ be unital $C^{*}$-algebras equipped with orthogonal filtrations. Assume that $G$ and $H$ are compact abelian groups acting respectively on $A$ and on $B$ in the filtration preserving way (so that they are objects of respective categories). Moreover, let $\chi: \hat{G} \times \hat{H} \rightarrow \mathbb{T}$ be a bicharacter. The coactions $\alpha_{A}: A \rightarrow A \otimes \mathrm{C}(G)$ and $\alpha_{B}: B \rightarrow B \otimes \mathrm{C}(H)$ define a canonical coaction $\gamma$ of $\mathrm{C}(K):=\mathrm{C}(G \times H)$ on $E:=A \otimes B$. Furthermore $\chi$ defines a bicharacter $\psi$ on $\hat{K}$ via the formula

$$
\psi: \hat{K} \times \hat{K} \rightarrow \mathbb{T}, \psi\left(\left(g_{1}, h_{1}\right),\left(g_{2}, h_{2}\right)\right)=\chi\left(g_{2}, h_{1}\right)^{-1}, \quad g_{1}, g_{2}, h_{1}, h_{2} \in \hat{K} .
$$

Since $\psi$ is a bicharacter, it defines a 2 -cocycle on the group $\hat{K}$. The Rieffel deformation of the data $(E, \gamma, \psi)$ yields a new unital $C^{*}$-algebra $E_{\psi}$.

By Theorem 6.2 of [35], $E_{\psi}$ is isomorphic to $A \bigotimes_{\psi} B$. Therefore, we can apply Theorem 5.4 to compute the quantum symmetry group of $E_{\psi}$. Concrete examples can be obtained in the following way, using the notions appearing in the next section: take $A$ and $B$ to be any two $C^{*}$ algebras appearing in Examples 6.6, 6.7, 6.8, 6.9 and $G=H=\mathbb{T}^{n}$. Then the homomorphism $f_{\lambda}$ of Proposition 6.5 defines the required bicharacter.

\section{The Case of the Reduced Crossed Products}

In this section we will apply the general results obtained before for quantum symmetry groups of twisted tensor products to the case of crossed products by discrete group actions.

6.1. Quantum symmetries of reduced crossed products. Throughout this subsection, we will adopt the following notations and conventions. The triple $\widetilde{A}:=\left(A, \tau_{A},\left\{A_{i}\right\}_{i \geq 0}\right)$ will denote an orthogonal filtration of a unital $C^{*}$-algebra $A$. Further, $\Gamma$ will denote a discrete countable group with a neutral element $e$ and a proper length function $l: \Gamma \rightarrow \mathbb{N}_{0}$, so that the $\mathrm{C}^{*}$-algebra $B:=\mathrm{C}_{\mathrm{r}}^{*}(\Gamma)$ has the orthogonal filtration $\widetilde{B}:=\left(\mathrm{C}_{\mathrm{r}}^{*}(\Gamma), \tau_{\Gamma},\left\{B_{n}^{l}\right\}_{n \geq 0}\right)$ as in Example 4.3. Symbols $\gamma_{A}^{\mathrm{u}}$ and $\gamma_{A}$ will denote respectively the universal and reduced version of the action of $\operatorname{QISO}(\widetilde{A})$ on $A$, and $\mathrm{W}^{\Gamma}$ will denote the reduced bicharacter associated to $\Gamma$ (see Theorem 2.13). Given any action $\beta$ of $\Gamma$ on $A$ (classically viewed as a homomorphism from $\Gamma$ to $\operatorname{Aut}(A)$, but here interpreted as a coaction of $\mathrm{C}_{0}(\Gamma)$, that is a morphism $\beta \in \operatorname{Mor}\left(A, A \otimes \mathrm{C}_{0}(\Gamma)\right)$ satisfying the Eq. (2.8)), we will denote the resulting reduced crossed product, contained in $\mathcal{M}\left(A \otimes \mathbb{K}\left(\ell^{2}(\Gamma)\right)\right)$, as $A \rtimes_{\beta, \mathrm{r}} \Gamma$. As customary, we will write then $a \lambda_{g}$ for $\beta(a)\left(1 \otimes \lambda_{g}\right)$, where $a \in A, g \in \Gamma$. Moreover we will write $\tau:=\tau_{A} \circ \tau^{\prime} \in S\left(A \rtimes_{\beta, \mathrm{r}} \Gamma\right)$, where $\tau^{\prime}$ is the canonical conditional expectation from $A \rtimes_{\beta, \mathrm{r}} \Gamma$ onto $A$ defined by the continuous linear extension of the prescription $\tau^{\prime}\left(\sum_{g} a_{g} \lambda_{g}\right)=a_{e}$. Finally, given the data as above define for each $i, j \geq 0$

$$
A_{i j}:=\operatorname{span}\left\{a_{i} \lambda_{g_{j}} \mid a_{i} \in A_{i}, l\left(g_{j}\right)=j\right\} .
$$

Note first the following easy lemma, extending Theorem 2.13.

Lemma 6.1. Let $\widetilde{A}, \widetilde{B}$ be as above and fix an action $\beta$ of $\Gamma$ on $A$. The isomorphism $\Psi: A \bigotimes_{\mathrm{V}}$ $B \rightarrow A \rtimes_{\beta, \mathrm{r}} \Gamma$, discussed in Theorem 2.13 has the following properties:

$$
\tau=\left(\tau_{A} \bigotimes_{\mathrm{W}^{\Gamma}} \tau_{\Gamma}\right) \circ \Psi
$$

and for each $i, j \geq 0$ we have

$$
\Psi\left(j_{A}\left(A_{i}\right) j_{B}\left(B_{j}^{l}\right)\right)=A_{i j} .
$$


Proof. Easy computation.

The above lemma shows that we have a natural candidate for an orthogonal filtration of the reduced crossed product. This raises two natural questions: first, when does the family $\left\{A_{i j}: i, j \geq 0\right\}$ indeed form an orthogonal filtration, and second, when can we determine the respective quantum symmetry group. The next theorem involves a condition which identifies the family $\left\{A_{i j}: i, j \geq 0\right\}$ as an orthogonal filtration arising via the construction described in Sect. 4.2, and further allows us to apply Theorem 5.1 to compute the quantum symmetry group in question. Later, in Proposition 6.13, we will see another, more general situation, under which $\left\{A_{i j}: i, j \geq 0\right\}$ still forms an orthogonal filtration. Then of course the second question will have to be addressed separately.

Theorem 6.2. Let $\widetilde{A}, \widetilde{B}$ be as above. Suppose that the map $\pi \in \operatorname{Mor}\left(\mathrm{C}^{\mathrm{u}}(\mathrm{QISO}(\widetilde{A}))\right.$, $\left.\mathrm{C}_{0}(\Gamma)\right)$ is a Hopf *-homomorphism, describing quantum group morphism from $\Gamma$ to $\operatorname{QISO}(\widetilde{A})$, and define the action $\beta \in \operatorname{Mor}\left(A, A \otimes \mathrm{C}_{0}(\Gamma)\right)$ as

$$
\beta:=(\mathrm{id} \otimes \pi) \circ \gamma_{A}^{\mathrm{u}} .
$$

Then the triplet $\left(A \rtimes_{\beta, \mathrm{r}} \Gamma, \tau,\left(A_{i j}\right)_{i, j \geq 0}\right)$ is an orthogonal filtration, denoted by $\widetilde{A} \rtimes_{\beta} \widetilde{B}$. Recall the Hopf ${ }^{*}$-homomorphism $\pi_{\Gamma} \in \operatorname{Mor}\left(\mathrm{C}(\mathrm{QISO}(\widetilde{B})), \mathrm{C}^{*}(\Gamma)\right)$ mentioned in Remark 4.7 and define a bicharacter $\left.\mathrm{V} \in \mathcal{U}\left(\mathrm{C}_{0}(\widehat{\mathrm{QISO}(\widetilde{A})}) \otimes \mathrm{C}_{0}(\widehat{\mathrm{QISO}(\widetilde{B}})\right)\right)$ as

$$
\mathrm{V}:=\left(\widehat{\pi} \otimes \widehat{\pi_{\Gamma}}\right)\left(\mathbb{W}^{\Gamma}\right),
$$

where $\widehat{\pi}, \widehat{\pi_{\Gamma}}$ denote the respective dual morphisms.

Then $\operatorname{QISO}\left(\widetilde{A} \rtimes_{\beta} \widetilde{B}\right) \cong \mathfrak{D}_{\mathrm{V}}$, where $\mathfrak{D}_{\mathrm{V}}$ is the Drinfeld double of $\mathrm{QISO}(\widetilde{A})$ and $\operatorname{QISO}(\widetilde{B})$ determined by $\mathrm{V}$.

Proof. The proof proceeds via identifying the sets $A_{i j}$ with those constructed via Proposition 4.9.

By virtue of Lemma 2.11 , the coactions $\beta$ and $\hat{\Delta}_{\Gamma}$ are induced by $\pi$ and $\pi_{\Gamma}$, respectively. Note that $\beta$ and $\hat{\Delta}_{\Gamma}$ are injective. Thus we can use [35, Theorem 5.2] to deduce there is an isomorphism $\Theta: A \otimes_{\mathrm{V}} \mathrm{C}_{\mathrm{r}}^{*}(\Gamma) \rightarrow A \bigotimes_{\mathrm{W}^{\Gamma}} \mathrm{C}_{\mathrm{r}}^{*}(\Gamma)$ such that

$$
\Theta\left(j_{A}^{\prime}\right)=j_{A}, \quad \Theta\left(j_{B}^{\prime}\right)=j_{B},
$$

where $j_{A}^{\prime}$ and $j_{B}^{\prime}$ are embeddings of $A$ and $\mathrm{C}_{\mathrm{r}}^{*}(\Gamma)$ into $A \bigotimes_{\mathrm{V}} \mathrm{C}_{\mathrm{r}}^{*}(\Gamma)$. An argument similar to that used in the proof of Corollary 4.10 shows that the isomorphism $\Theta$ maps $\tau_{A} \otimes_{\mathrm{V}} \tau_{\Gamma}$ and $\left\{j_{A}^{\prime}\left(A_{i}\right) j_{B}^{\prime}\left(B_{j}\right)\right\}_{i, j \geq 0}$ to $\tau_{A} \bigotimes_{\mathrm{W}^{\Gamma}} \tau_{\Gamma}$ and $\left\{j_{A}\left(A_{i}\right) j_{B}\left(B_{j}\right)\right\}_{i, j \geq 0}$, respectively. Then, using Lemma 6.1, we obtain that $\Psi \circ \Theta$ maps $\tau_{A} \bigotimes_{\mathrm{V}} \tau_{\Gamma}$ and $\left\{j_{A}^{\prime}\left(A_{i}\right) j_{B}^{\prime}\left(B_{j}\right)\right\}_{i, j \geq 0}$ to $\tau$ and $\left\{A_{i j}\right\}_{i, j \geq 0}$, respectively. The map $\Psi \circ \Theta$ is an isomorphism and the triplet $\left(A \bigotimes_{\mathrm{V}} B, \tau_{A} \bigotimes_{\mathrm{V}} \tau_{\Gamma},\left\{j_{A}^{\prime}\left(A_{i}\right) j_{B}^{\prime}\left(B_{j}\right)\right\}_{i, j \geq 0}\right)$ is an orthogonal filtration by Proposition 4.9. Hence, the triplet $\left(A \rtimes_{\beta, \mathrm{r}} \Gamma, \tau,\left(A_{i j}\right)_{i, j \geq 0}\right)$ is also an orthogonal filtration of $A \rtimes_{\beta, \mathrm{r}} \Gamma$.

Then Theorem 5.1 allows us to conclude the proof.

We quickly note that the theorem applies of course to the case of the trivial action.

Corollary 6.3. Let $\widetilde{A}, \widetilde{B}$ be the filtrations introduced above. Let $\beta$ denote the trivial action of $\Gamma$ on $A$. Then $\operatorname{QISO}(\widetilde{A} \otimes \widetilde{B})$ is isomorphic to $\operatorname{QISO}(\widetilde{A}) \times \operatorname{QISO}(\widetilde{B})$.

Proof. It suffices to recall that for a trivial action $\beta, A \rtimes_{\beta, \mathrm{r}} \Gamma$ is isomorphic with $A \otimes \mathrm{C}_{\mathrm{r}}^{*}(\Gamma)$ and apply Corollary 5.5. 
Remark 6.4. Note that the action defined by the formula (6.2) preserves the state $\tau_{A}$. As we will see below, this preservation alone guarantees that the family $\left\{A_{i j}: i, j \geq 0\right\}$ forms an orthogonal filtration. The example presented in the last part of this section will show however that if we only assume that $\beta$ preserves $\tau_{A}$, then in general the quantum symmetry group need not be of the form discussed above. More specifically we will prove that the quantum symmetry group $\operatorname{QISO}\left(\widetilde{A} \rtimes_{\beta} \widetilde{B}\right)$ need not be a Drinfeld double of $\operatorname{QISO}(\widetilde{A})$ and $\operatorname{QISO}(\widetilde{B})$ with respect to any bicharacter.

6.2. Examples. In this subsection we present several examples illustrating the scope of Theorem 6.2 and also discuss the cases in which it does not apply. We begin with the following observation, presenting a general situation where one can apply Theorem 6.2.

Proposition 6.5. Suppose that $\tilde{A}:=\left(A, \tau_{A},\left(\underset{\widetilde{A}}{A_{i}}\right)_{i \geq 0}\right)$ is an orthogonal filtration and denote as usual by $\gamma_{A}^{\mathrm{u}}$ the coaction of $\mathrm{C}^{\mathrm{u}}(\mathrm{QISO}(\widetilde{A}))$ on $A$. Suppose that $n \in \mathbb{N}$ and we have a quantum group morphism from $\mathbb{T}^{n}$ to $\mathrm{QISO}(\widetilde{A})$, described by a Hopf*-homomorphism $\pi \in \operatorname{Mor}\left(\mathrm{C}^{\mathrm{u}}(\mathrm{QISO}(\widetilde{A})), \mathrm{C}\left(\mathbb{T}^{n}\right)\right)$. Further let $\lambda:=\left(\lambda_{1}, \lambda_{2}, \ldots, \lambda_{n}\right) \in \mathbb{T}^{n}$ and define $a$ homomorphism $f_{\lambda}: \mathbb{Z}^{n} \rightarrow \mathbb{T}^{n}$ by the formula $f_{\lambda}\left(m_{1}, m_{2}, \ldots, m_{n}\right)=\lambda_{1}^{m_{1}} \cdots \lambda_{n}^{m_{n}}$ and let $f_{\lambda}^{*}: \mathrm{C}\left(\mathbb{T}^{n}\right) \rightarrow \mathrm{C}_{0}\left(\mathbb{Z}^{n}\right)$ denote the associated Hopf ${ }^{*}$-homomorphism.

Then the formula $\beta:=\left(\mathrm{id} \otimes f_{\lambda}^{*} \circ \pi\right) \circ \gamma_{A}^{\mathrm{u}}$ defines an action $\beta$ of $\Gamma:=\mathbb{Z}^{n}$ on $A$ and the $\mathrm{C}^{*}$-algebra $A \rtimes_{\beta, \mathrm{r}} \mathbb{Z}^{n}$ satisfies the conditions of Theorem 6.2.

Proposition 6.5 can be directly applied to the following class of examples.

Example 6.6. Let $M$ be a compact Riemannian manifold. Assume that $\mathbb{T}^{n}$ is a subgroup of the maximal torus of the isometry group $\operatorname{ISO}(M)$ for some $n \geq 1$. Consider the orthogonal filtration $\left(E, \tau,\left\{V_{i}\right\}_{i}, J, W\right)$ on $\mathrm{C}(M)$ coming from the Hodge-Dirac operator $d+d^{*}$ (see Example 2.5.(1) of [18]). It is well known that (Remark 2.13 of [18]) ISO $(M)$ is an object of the category $\mathcal{C}\left(E, \tau,\left\{V_{i}\right\}_{i}, J, W\right)$ and thus we have a quantum group morphism from $\mathbb{T}^{n}$ to $\operatorname{QISO}\left(E, \tau,\left\{V_{i}\right\}_{i}, J, W\right)$. Now we can apply Proposition 6.5.

In particular, if we take $M=\mathbb{T}^{n}$, the resulting crossed product is the $2 n$-dimensional noncommutative torus.

Example 6.7. For $q \in(0,1)$ and $G$ a compact semisimple Lie group, let $A:=\mathrm{C}\left(G_{q}\right)$ denote the reduced version of the $q$-deformation of $G$. It is well known that $G_{q}$ is coamenable. Consider the (reduced, ergodic) action of $G_{q}$ on itself, i.e. the coproduct $\Delta: A \rightarrow A \otimes A$. Then the faithful Haar state $\tau_{A}$ of $A$ is the unique invariant state for the action $\Delta$. For an irreducible representation $\pi$ of $G_{q}$ denote by $A_{\pi}$ the linear span of its matrix coefficients. By [8, Theorem 3.6], we have an orthogonal filtration $\widetilde{A}:=\left(A, \tau_{A},\left\{A_{\pi}\right\}_{\pi \in \operatorname{Irr}\left(G_{q}\right)}\right)$ such that $\left(\mathrm{C}\left(G_{q}\right), \Delta\right)$ is an object of $\mathcal{C}(\widetilde{A})$. Therefore, we have a Hopf *-homomorphism $f: \mathrm{C}^{\mathrm{u}}(\mathrm{QISO}(\tilde{A})) \rightarrow \mathrm{C}\left(G_{q}\right)$. Let us recall that the maximal toral subgroup $\mathbb{T}^{n}$ of $G$ is still a quantum subgroup of $G_{q}$, so that we have a Hopf *-homomorphism $g: \mathrm{C}\left(G_{q}\right) \rightarrow \mathrm{C}\left(\mathbb{T}^{n}\right)$. Thus we obtain a Hopf ${ }^{*}$-homomorphism from $\mathrm{C}^{\mathrm{u}}(\mathrm{QISO}(\widetilde{A}))$ to $\mathrm{C}\left(\mathbb{T}^{n}\right)$ and we end up in the framework of Proposition 6.5 .

Example 6.8. The situation described above can be generalized to quantum homogeneous spaces of $G_{q}$ (we continue using the same notations as above). Let $H$ be a quantum subgroup of $G_{q}$, given by the surjective Hopf *-homomorphism $\chi: \mathrm{C}\left(G_{q}\right) \rightarrow \mathrm{C}(H)$. Let $\mathrm{C}\left(G_{q} / H\right):=\left\{a \in \mathrm{C}\left(G_{q}\right) \mid(\chi \otimes \mathrm{id})(\Delta(a))=1 \otimes a\right\}$. We then have a reduced ergodic coaction $\left.\Delta\right|_{\mathrm{C}\left(G_{q} / H\right)}: \mathrm{C}\left(G_{q} / H\right) \rightarrow \mathrm{C}\left(G_{q} / H\right) \otimes \mathrm{C}\left(G_{q}\right)$, and an orthogonal filtration of $\mathrm{C}\left(G_{q} / H\right)$ resulting from an application of Theorem 3.6 of [8]. The analogous 
argument to that in Example 6.7 shows that the conditions of Proposition 6.5 hold also here.

Example 6.9. This example deals with the orthogonal filtrations of Cuntz algebras constructed in Proposition 4.5 in [24]. Let $N \in \mathbb{N}$, and let $\mathcal{O}_{N}$ be the associated Cuntz algebra. We will denote the canonical generators of $\mathcal{O}_{N}$ by the symbols $S_{1}, \ldots, S_{N}$. For a multi-index $\boldsymbol{\mu}=\left(\mu_{1}, \mu_{2}, \ldots, \mu_{k}\right)$, where $k \in \mathbb{N}$ and $\mu_{i} \in\{1,2, \ldots, N\}$, we write $S_{\boldsymbol{\mu}}$ for $S_{\mu_{1}} \cdots S_{\mu_{k}}$ and $|\boldsymbol{\mu}|$ for $\sum_{i=1}^{k} \mu_{i}$. We have the gauge action $\gamma$ of $\mathbb{T}$ on $\mathcal{O}_{N}$ defined by the equation $\gamma_{z}\left(S_{i}\right)=z S_{i}$ where $z \in \mathbb{T}, i=1, \ldots, N$. Then the fixed point algebra $\mathcal{O}_{N}^{\gamma}$ has a natural filtration $\left\{W_{k}\right\}_{k \geq 0}$. The authors of [24] construct further an orthogonal filtration $\left\{V_{k, m}\right\}_{k, m \geq 0}$ of $\mathcal{O}_{N}$ with respect to the canonical state $\omega$. It can be easily seen that the filtration is given by the following formulas $(k \geq 0)$ :

$$
\begin{aligned}
V_{k, 0} & =W_{k}, V_{k, m}=\operatorname{Span}\left\{S_{\mu} x: x \in W_{k},|\mu|=m\right\} \text { for } m>0, \\
V_{k, m} & =\operatorname{Span}\left\{x S_{\mu}^{*}: x \in W_{k},|\mu|=-m\right\} \text { for } m<0
\end{aligned}
$$

Suppose we have an action of $\mathbb{T}^{n}$ on $\mathcal{O}_{N}$ which acts on each $S_{i}$ merely by scalar multiplication. We claim that Proposition 6.5 applies to such actions. Indeed, it is easy to see that $\mathbb{T}^{n}$ acts on $\mathcal{O}_{N}$ by quantum symmetries. Therefore, we have a quantum group morphism from $\mathbb{T}^{n}$ to QISO $\left(\widetilde{\mathcal{O}_{N}}\right)$ and we can apply Proposition 6.5 to obtain an action of $\mathbb{Z}^{n}$ on $\mathcal{O}_{N}$ satisfying the conditions in Theorem 6.2.

The first example of such a group action is of course the gauge action $\gamma$ of $\mathbb{T}$ defined above. More generally, following Katsura's prescription in Definition 2.1 of [26], we have actions of $\mathbb{T}^{n}$ on $\mathcal{O}_{N}$ defined by

$$
\beta_{z}^{\chi}\left(S_{i}\right)=\chi(z) S_{i}, \quad i=1, \ldots, N, z \in \mathbb{T}^{n},
$$

$\chi$ being a fixed character of $\mathbb{T}^{n}$ (so an element of $\mathbb{Z}^{n}$ ).

Then we can apply Proposition 6.5 as mentioned above.

6.3. The example of the Bunce-Deddens algebra. This subsection deals with the quantum symmetries of the Bunce-Deddens algebra. Let us recall that the Bunce-Deddens algebra is isomorphic to the crossed product $A \rtimes_{\beta} \mathbb{Z}$, where $A$ is the commutative AF algebra of continuous functions on the middle-third Cantor set and $\beta$ is the odometer action on $A$. More precisely, $A$ is an AF algebra arising as the limit of the unital embeddings

$$
\mathbb{C}^{2} \longrightarrow \mathbb{C}^{2} \otimes \mathbb{C}^{2} \longrightarrow \mathbb{C}^{2} \otimes \mathbb{C}^{2} \otimes \mathbb{C}^{2} \longrightarrow \cdots
$$

Let us recall a multi-index notation for a basis of $\mathcal{A}_{n}$ (the $n$-th element of the above sequence), as introduced in [10]. For each $n \in \mathbb{N}, \mathcal{J}_{n}$ will denote the set $\left\{i_{1} i_{2} \cdots i_{n}\right.$ : $i_{j} \in\{1,2\}$ for $\left.j=1, \ldots, n\right\}$. Multi-indices in $\mathcal{J}:=\bigcup_{n \in \mathbb{N}} \mathcal{J}_{n}$ will be denoted by capital letters $I, J, \ldots$ and we let the canonical basis of the algebra $\mathcal{A}_{n}$ built of minimal projections be indexed by elements of $\mathcal{J}_{n}$. Hence, the basis vectors of $\mathcal{A}_{n}$ will be denoted by $e_{I}$, where $I$ belongs to $\mathcal{J}_{n}$.

Then the natural embeddings $i_{n}: \mathcal{A}_{n} \rightarrow \mathcal{A}_{n+1}$ can be described by the formula

$$
i_{n}\left(e_{I}\right)=e_{I 1}+e_{I 2}, \quad I \in \mathcal{J}_{n},
$$

where we use the standard concatenation of multi-indices. 
The quantum isometry group (in the sense of [9]) of the $\mathrm{C}^{*}$-algebra $A$ was studied in [10]. For more details, we refer to Chapter 5 of [21]. Indeed, we can fix a spectral triple on $A$ coming from the family constructed by Christensen and Ivan [14]. It turns out (Theorem 3.1 of [10]) that the relevant quantum isometry group of $A$ (with respect to that triple), which we will denote $\mathbb{S}_{\infty}$, is the projective limit of the quantum isometry groups $\mathbb{S}_{n}$ of the finite dimensional commutative $C^{*}$-algebras $\mathcal{A}_{n}$, equipped with suitable spectral triples (or, equivalently, viewed as algebras of functions on respective finite Bratteli diagrams, see [10]). More precisely, the algebras $\mathrm{C}^{\mathrm{u}}\left(\mathbb{S}_{n}\right)$ are inductively defined by the formulas

$$
\mathrm{C}^{\mathrm{u}}\left(\mathbb{S}_{1}\right)=\mathrm{C}\left(\mathbb{Z}_{2}\right), \quad \mathrm{C}^{\mathrm{u}}\left(\mathbb{S}_{n+1}\right)=\mathrm{C}^{\mathrm{u}}\left(\mathbb{S}_{n}\right) * \mathrm{C}^{\mathrm{u}}\left(\mathbb{S}_{n}\right) \oplus \mathrm{C}^{\mathrm{u}}\left(\mathbb{S}_{n}\right) * \mathrm{C}^{\mathrm{u}}\left(\mathbb{S}_{n}\right), \quad n \in \mathbb{N} .
$$

Let us introduce some more notation, following [10]. The algebra $\mathrm{C}^{\mathrm{u}}\left(\mathbb{S}_{n+1}\right)$ is generated by elements $\left\{a_{K l, I j}: K, I \in \mathcal{J}_{n}, l, j=1,2\right\}$, and the coaction $\gamma_{n+1}^{\mathrm{u}}$ of $\mathrm{C}^{\mathrm{u}}\left(\mathbb{S}_{n+1}\right)$ on $\mathcal{A}_{n+1}$ is given by the following formula:

$$
\gamma_{n+1}^{\mathrm{u}}\left(e_{I j}\right)=\sum_{K \in \mathcal{J}_{n}, l=1,2} e_{K l} \otimes a_{K l, I j}, \quad I \in \mathcal{J}_{n}, j \in\{1,2\} .
$$

The sequence $\left(\mathrm{C}^{\mathrm{u}}\left(\mathbb{S}_{n}\right)\right)_{n \in \mathbb{N}}$ defines an inductive system with connecting Hopf *-homomorphisms $\phi_{n, m}: \mathrm{C}^{\mathrm{u}}\left(\mathbb{S}_{n}\right) \rightarrow \mathrm{C}^{\mathrm{u}}\left(\mathbb{S}_{m}\right)$ (if $n \leq m$ ) satisfying the equation

$$
\phi_{n k}=\phi_{m k} \circ \phi_{n m}, \quad n<m<k .
$$

Further, one can check that for all $n \in \mathbb{N}$ we have

$$
\left(i_{n} \otimes \phi_{n, n+1}\right) \circ \gamma_{n}^{\mathrm{u}}=\gamma_{n+1}^{\mathrm{u}} \circ i_{n} .
$$

Combining (6.3) and (6.5), we see that for all $n \in \mathbb{N}, I, J \in \mathcal{J}_{n}$,

$$
\phi_{n, n+1}\left(a_{J, I}\right)=a_{J 1, I 1}+a_{J 1, I 2}=a_{J 2, I 1}+a_{J 2, I 2} .
$$

Thus, we have a compact quantum group $\mathbb{S}_{\infty}$ arising as a projective limit of this system, a compact quantum group morphism from $\mathbb{S}_{\infty}$ to $\mathbb{S}_{n}$ represented by a Hopf ${ }^{*}$-homomorphism $\phi_{n, \infty} \in \operatorname{Mor}\left(\mathrm{C}^{\mathrm{u}}\left(\mathbb{S}_{n}\right), \mathrm{C}^{\mathrm{u}}\left(\mathbb{S}_{\infty}\right)\right)$ and a canonical coaction $\gamma^{\mathrm{u}} \in \operatorname{Mor}(A$, $\left.A \otimes \mathrm{C}^{\mathrm{u}}\left(\mathbb{S}_{\infty}\right)\right)$.

Let $\omega_{\xi}$ denote the canonical trace on $A$. As explained in Subsection 3.3 of [8], $A$ can be equipped with an orthogonal filtration $\left(A, \omega_{\xi},\left\{\mathcal{A}_{n} \backslash \mathcal{A}_{n-1}\right\}_{n \in \mathbb{N}}\right)$. As a result, by Theorem 3.2 of [8], the quantum symmetry group of $\left(A, \omega_{\xi},\left\{\mathcal{A}_{n} \backslash \mathcal{A}_{n-1}\right\}_{n}\right)$ is isomorphic to $\mathbb{S}_{\infty}$.

Now, let us recall the odometer action $\beta$ on $A$. In fact the action $\beta$ arises as an inductive limit of actions $\beta_{n}$ of $\Gamma_{n}:=\mathbb{Z}_{2^{n}}$ on $\mathcal{A}_{n}$. Let $\mathbf{I}_{n}$ denote the element $(111 \cdots 1) \in \mathcal{J}_{n}$.

We first define inductively homomorphisms $\sigma_{n}: \Gamma_{n} \rightarrow \operatorname{Aut}\left(\mathcal{J}_{n}\right), n \in \mathbb{N}$. We do it as follows, for simplicity writing $\sigma_{n}^{i}$ for $\sigma_{n}(i)$ : first the map $\sigma_{1}: \mathcal{J}_{1} \rightarrow \mathcal{J}_{1}$ is defined as

$$
\sigma_{1}(1)=2, \sigma_{1}(2)=1 \text {. }
$$

In the inductive step, for each $j=1,2$ and $0 \leq i \leq 2^{n}-1$, the map $\sigma_{n+1}^{i}: \mathcal{J}_{n+1} \rightarrow \mathcal{J}_{n+1}$ is defined by

$$
\begin{aligned}
& \sigma_{n+1}^{i}\left(\sigma_{n}^{k}\left(\mathbf{I}_{n}\right) j\right)=\sigma_{n}^{k+i}\left(\mathbf{I}_{n}\right) j \quad \text { if } \quad 0 \leq k+i \leq 2^{n}-1, \quad j=1,2, \\
& \sigma_{n+1}^{i}\left(\sigma_{n}^{k}\left(\mathbf{I}_{n}\right) j\right)=\sigma_{n}^{k+i}\left(\mathbf{I}_{n}\right)(j+1) \quad \text { if } \quad k+i \geq 2^{n}, \quad j=1,2,
\end{aligned}
$$


where $j+1$ is defined mod 2 and we have used the concatenation of indices. It is clear that $\mathcal{J}_{n}=\left\{\sigma_{n}^{i}\left(\mathbf{I}_{n}\right): i=0,1,2, \ldots, 2^{n}-1\right\}$.

Let $\left\{\delta_{j}: j=0,1,2 \ldots, 2^{n-1}\right\}$ denote the standard basis of the finite-dimensional (commutative) algebra $\mathrm{C}\left(\Gamma_{n}\right)$. Then for each $n \in \mathbb{N}$ the coaction $\beta_{n} \in \operatorname{Mor}\left(\mathcal{A}_{n}, \mathcal{A}_{n} \otimes\right.$ $\left.\mathrm{C}\left(\Gamma_{n}\right)\right)$ is defined as

$$
\beta_{n}\left(e_{\sigma_{n}^{i}\left(\mathbf{I}_{n}\right)}\right)=\sum_{j=0}^{2^{n}-1} e_{\sigma_{n}^{j}\left(\mathbf{I}_{n}\right)} \otimes \delta_{j-i} .
$$

We then easily check that we have an inductive system $\left(\mathrm{C}\left(\Gamma_{n}\right), \beta_{n}\right)_{n \in \mathbb{N}}$ of coactions, with connecting morphisms $\psi_{n, m} \in \operatorname{Mor}\left(\mathrm{C}\left(\Gamma_{n}\right), \mathrm{C}\left(\Gamma_{m}\right)\right.$ ) (for $n<m$ ) such that for all $n, m, k \in \mathbb{N}, n<m<k, \psi_{n, k}=\psi_{m, k} \circ \psi_{n, m}$, and a Hopf *-homomorphism $\psi_{n, \infty} \in \operatorname{Mor}\left(\mathrm{C}\left(\Gamma_{n}\right), \mathrm{C}_{0}(\mathbb{Z})\right)$. In particular for $n \in \mathbb{N}$ the map $\psi_{n, n+1}$ is given by

$$
\psi_{n, n+1}\left(\delta_{j}^{\Gamma_{n}}\right)=\delta_{j}^{\Gamma_{n+1}}+\delta_{2^{n}+j}^{\Gamma_{n+1}}, \quad j=0, \ldots, 2^{n}-1 .
$$

Thus, finally we can define the odometer action using the universal property of the inductive limit, so that for all $n \in \mathbb{N}$

$$
\beta \circ \phi_{n, \infty}:=\left(\mathrm{id} \otimes \psi_{n, \infty}\right) \circ \beta_{n} .
$$

In what follows, we will replace the symbols $\sigma_{n}^{i}$ and $\mathbf{I}_{n}$ by $\sigma^{i}$ and $\mathbf{I}$, respectively, unless there is any risk of confusion.

Thus, we have an orthogonal filtration $\widetilde{A}:=\left(A, \omega_{\xi},\left\{\mathcal{A}_{n} \backslash \mathcal{A}_{n-1}\right\}_{n \in \mathbb{N}}\right)$ of a unital $\mathrm{C}^{*}$-algebra $A$ and an action $\beta$ of $\mathbb{Z}$ on $A$. In order to apply Theorem 6.2, we need a Hopf $*$-homomorphism $\pi \in \operatorname{Mor}\left(\mathrm{C}^{\mathrm{u}}(\mathrm{QISO}(\widetilde{A})), C_{0}(\mathbb{Z})\right)$ such that $\beta=(\mathrm{id} \otimes \pi) \circ \gamma_{A}^{\mathrm{u}}$.

In order to define $\pi$, we begin by defining a quantum group homomorphism from $\mathrm{C}^{\mathrm{u}}\left(\mathbb{S}_{n}\right)$ to $\mathrm{C}\left(\Gamma_{n}\right)$.

Lemma 6.10. Let $n \in \mathbb{N}$. Define $\pi_{n} \in \operatorname{Mor}\left(\mathrm{C}^{\mathrm{u}}\left(\mathbb{S}_{n}\right), \mathrm{C}\left(\Gamma_{n}\right)\right)$ by the formula

$$
\pi_{n}\left(a_{\sigma^{i}(\mathbf{I}), \sigma^{j}(\mathbf{I})}\right)=\delta_{i-j}, \quad i, j=1, \ldots, 2^{n} .
$$

Then $\pi_{n}$ is a Hopf ${ }^{*}$-homomorphism and

$$
\left(\mathrm{id} \otimes \psi_{n, \infty} \circ \pi_{n}\right) \circ \gamma_{n}^{\mathrm{u}}=\beta \circ \phi_{n, \infty} .
$$

Proof. To prove that $\pi_{n}$ is a Hopf *-homomorphism, we need to recall the universal property of the quantum group $\mathbb{S}_{n}$. Let us begin by recalling that both $\mathbb{S}_{n+1}$ and $\Gamma_{n+1}$ are quantum subgroups of the quantum permutation group $S_{2^{n+1}}^{+}$. Indeed, this follows from [46] since both $\mathbb{S}_{n+1}$ and $\Gamma_{n+1}$ are compact quantum groups acting faithfully on $A_{n+1} \approx \mathbb{C}^{2^{n+1}}$. In particular, the elements $\left\{a_{I J}: I, J \in \mathcal{J}_{n+1}\right\}$ satisfy the magic unitary conditions of the canonical generators of $\mathrm{C}\left(S_{2^{n+1}}^{+}\right)$. The only extra conditions on $a_{I J}$ are dictated by the following equalities (see equation (2.1) of [10]):

$$
\gamma_{n+1}^{\mathrm{u}}\left(i_{n}\left(A_{n}\right)\right) \subseteq i_{n}\left(A_{n}\right) \otimes \mathrm{C}^{\mathrm{u}}\left(\mathbb{S}_{n+1}\right) .
$$

Therefore, if $\delta$ is an action of a compact quantum group $H$ on $A_{n+1}$ satisfying the condition

$$
\delta\left(i_{n}\left(A_{n}\right)\right) \subseteq i_{n}\left(A_{n}\right) \otimes \mathrm{C}^{\mathrm{u}}(H),
$$

then we have a quantum group morphism from $H$ to $\mathbb{S}_{n}$ given by a map $\Phi \in \operatorname{Mor}\left(\mathrm{C}^{\mathrm{u}}\left(\mathbb{S}_{n}\right)\right.$, $\left.\mathrm{C}^{\mathrm{u}}(H)\right)$ such that $(\mathrm{id} \otimes \Phi) \circ \gamma_{n+1}^{\mathrm{u}}=\delta$. We claim that the action $\beta_{n+1}$ of $\Gamma_{n+1}$ on 
$A_{n+1}$ satisfies the displayed condition (6.12). Indeed, by (6.3), (6.7) and (6.8), for all $0 \leq i \leq 2^{n}-1$ we have

$$
\begin{aligned}
\beta_{n+1} & \left(i_{n}\left(e_{\sigma^{i}\left(\mathbf{I}_{n}\right)}\right)\right) \\
= & \beta_{n+1}\left(e_{\sigma^{i}(\mathbf{I}) 1}\right)+\beta_{n+1}\left(e_{\sigma^{i}(\mathbf{I}) 2}\right) \\
= & \sum_{j=0}^{2^{n}-1}\left[e_{\sigma^{j}(\mathbf{I}) 1} \otimes \delta_{j-i}+e_{\sigma^{j}(\mathbf{I}) 2} \otimes \delta_{j-i+2^{n}}+e_{\sigma^{j}(\mathbf{I}) 2} \otimes \delta_{j-i}+e_{\sigma^{j}(\mathbf{I}) 1} \otimes \delta_{j-i+2^{n}}\right] \\
= & \sum_{j=0}^{2^{n}-1}\left(e_{\sigma^{j}(\mathbf{I}) 1}+e_{\sigma^{j}(\mathbf{I}) 2}\right) \otimes\left(\delta_{j-i}+\delta_{j-i+2^{n}}\right) \\
= & \sum_{j=0}^{2^{n}-1} i_{n}\left(e_{\sigma^{j}(\mathbf{I})}\right) \otimes\left(\delta_{j-i}+\delta_{j-i+2^{n}}\right),
\end{aligned}
$$

which proves our claim.

Finally, since (id $\left.\otimes \pi_{n}\right) \circ \gamma_{n}^{\mathrm{u}}=\beta_{n}$ holds, we have

$$
\left(\mathrm{id} \otimes \psi_{n, \infty} \circ \pi_{n}\right) \circ \gamma_{n}^{\mathrm{u}}=\left(\mathrm{id} \otimes \psi_{n, \infty}\right) \circ \beta_{n}=\beta \circ \phi_{n, \infty} .
$$

We naturally need also some compatibility conditions for the morphisms introduced in the last lemma.

Lemma 6.11. For each $n \in \mathbb{N}$ the following equality holds:

$$
\psi_{n, \infty} \circ \pi_{n}=\psi_{n+1, \infty} \circ \pi_{n+1} \circ \phi_{n, n+1} .
$$

Proof. We fix $n \in \mathbb{N}, j, k \in\left\{0, \ldots, 2^{n}-1\right\}$ and compute

$$
\begin{aligned}
& \left(\psi_{n+1, \infty} \circ \pi_{n+1} \circ \phi_{n, n+1}\right)\left(a_{\sigma^{j}(\mathbf{I}), \sigma^{k}(\mathbf{I})}\right) \\
& \quad=\left(\psi_{n+1, \infty} \circ \pi_{n+1}\right)\left(a_{\sigma^{j}(\mathbf{I}) 1, \sigma^{k}(\mathbf{I}) 1}+a_{\sigma^{j}(\mathbf{I}) 1, \sigma^{k}(\mathbf{I}) 2}\right) \\
& \quad=\psi_{n+1, \infty}\left(\delta_{(j-k)}+\delta_{\left(2^{n}+j-k\right)}\right) \\
& \quad=\left(\psi_{n+1, \infty} \circ \beta_{n, n+1}\right)\left(\delta_{j-k}\right) \\
& \quad=\psi_{n, \infty}\left(\delta_{j-k}\right) \\
& \quad=\left(\psi_{n, \infty} \circ \pi_{n}\right)\left(a_{\sigma^{j}(\mathbf{I}), \sigma^{k}(\mathbf{I})}\right)
\end{aligned}
$$

(by Lemma 6.10).

We are ready for the final statement, which implies that the approach of Theorem 6.2 can be used to produce an orthogonal filtration of the Bunce-Deddens algebra.

Proposition 6.12. There exists a Hopf ${ }^{*}$-homomorphism $\eta \in \operatorname{Mor}\left(\mathrm{C}^{\mathrm{u}}\left(\mathbb{S}_{\infty}\right), \mathrm{C}_{0}(\mathbb{Z})\right)$ such that

$$
(\mathrm{id} \otimes \eta) \circ \gamma^{\mathrm{u}}=\beta \text {. }
$$

Proof. For each $n \in \mathbb{N}$ put $\eta_{n}:=\psi_{n, \infty} \circ \pi_{n}: \operatorname{Mor}\left(\mathrm{C}^{\mathrm{u}}\left(\mathbb{S}_{n}\right), \mathrm{C}_{0}(\mathbb{Z})\right)$. Then the existence of the $\mathrm{C}^{*}$-homomorphism $\eta$ follows from standard properties of inductive limits once we apply Lemma 6.11. Since each $\eta_{n}$ is a Hopf*-homomorphism, it can be easily checked that so is $\eta$. Finally, the equation (id $\otimes \eta$ ) $\circ \gamma^{\mathrm{u}}=\beta$ follows from (6.11). 
6.4. A counterexample. Finally, as announced earlier, we show that the conditions of Theorem 6.2 can be weakened if we are only interested in the existence of an orthogonal filtration, but are actually necessary to obtain the form of the quantum symmetry group appearing in that theorem. Let us start by pointing out that a reduced crossed product can have an orthogonal filtration under more general conditions than those assumed in Theorem 6.2.

Proposition 6.13. Let $\widetilde{A}:=\left(A, \tau_{A},\left(A_{i}\right)_{i \geq 0}\right)$ be an orthogonal filtration. Suppose $\Gamma$ is a finitely generated discrete group having an action $\beta$ on $A$ such that $\tau_{A}\left(\beta_{g}(a)\right)=\tau_{A}(a)$ for all $a$ in $A, g \in \Gamma$. Then the triplet $\widetilde{A} \rtimes_{\beta} \widetilde{B}:=\left(A \rtimes_{\beta, \mathrm{r}} \Gamma, \tau,\left(A_{i j}\right)_{i, j \geq 0}\right)$, with $A_{i j}$ given via (6.1) defines an orthogonal filtration of the $\mathrm{C}^{*}$-algebra $A \rtimes_{\beta, \mathrm{r}} \Gamma$.

Proof. First observe that $A_{00}=\mathbb{C} .1$ and $\operatorname{Span}\left(\cup_{i, j \geq 0} A_{i j}\right)$ is a dense $*_{\text {-subspace of the }}$ $\mathrm{C}^{*}$-algebra $A \rtimes_{\beta, \mathrm{r}} \Gamma$. Moreover, it can be easily checked that $\tau$ is faithful on $A \rtimes_{\beta, \mathrm{r}} \Gamma$ using the faithfulness of $\tau_{A}$ and the canonical faithful conditional expectation $\tau^{\prime}: A \rtimes_{\beta, \mathrm{r}} \Gamma \rightarrow$ A.

Now let $i, j, p, q \geq 0,(i, j) \neq(p, q)$ and consider $a \in A_{i j}$ and $b \in A_{p q}$. We need to prove that $\tau\left(a^{*} b\right)=0$. By linearity it suffices to assume that $a=a_{i} \lambda_{\gamma_{1}}$ and $b=b_{p} \lambda_{\gamma_{2}}$ with $a_{i} \in A_{i}, b_{p} \in A_{p}, l\left(\gamma_{1}\right)=j$ and $l\left(\gamma_{2}\right)=q$. We then obtain

$$
\begin{aligned}
\tau\left(a^{*} b\right) & =\tau\left(\lambda_{\gamma_{1}^{-1}} a_{i}^{*} b_{p} \lambda_{\gamma_{2}}\right)=\tau\left(\beta_{\gamma_{1}^{-1}}\left(a_{i}^{*} b_{p}\right) \lambda_{\gamma_{1}^{-1} \gamma_{2}}\right) \\
& =\delta_{\gamma_{1}, \gamma_{2}} \tau_{A}\left(\beta_{\gamma_{1}^{-1}}\left(a_{i}^{*} b_{p}\right)\right)=\delta_{\gamma_{1}, \gamma_{2}} \tau_{A}\left(a_{i}^{*} b_{p}\right)=\delta_{j q} \delta_{i p} \delta_{\gamma_{1}, \gamma_{2}} \tau_{A}\left(a_{i}^{*} b_{p}\right)=0 .
\end{aligned}
$$

Thus the triplet $\left(A \rtimes_{\beta, \mathrm{r}} \Gamma, \tau,\left(A_{i j}\right)_{i, j \geq 0}\right)$ defines an orthogonal filtration of the $\mathrm{C}^{*}$-algebra $A \rtimes_{\beta, \mathrm{r}} \Gamma$.

Remark 6.14. A sufficient condition for the condition $\tau_{A}\left(\beta_{g}(a)\right)=\tau_{A}(a)$ to hold is that $\beta_{g}\left(A_{i}\right) \subseteq A_{i}$ for all $g \in \Gamma, i \geq 0$. However, this is not a necessary condition as the next example shows.

For the rest of the section we will consider an example where $A=\mathrm{C}^{*}\left(\mathbb{Z}_{9}\right)$, and $\Gamma=\mathbb{Z}_{3}$. The elements of each of these cyclic groups will be denoted by $\overline{0}, \overline{1}, \overline{2}$, and so on. We fix the (symmetric) generating sets on $\mathbb{Z}_{3}$ and $\mathbb{Z}_{9}$, respectively $\{\overline{1}, \overline{2}\}$ and $\{\overline{1}, \overline{8}\}$, so that each of the $\mathrm{C}^{*}$-algebras in question is equipped with the orthogonal filtration given by the word-length function associated with the corresponding generating set, as in Example 4.3. Let $\phi$ be an automorphism of $\mathbb{Z}_{9}$ of order 3, given by the formula $\phi(n)=4 n$ for $n \in \mathbb{Z}_{9}$. It induces an action of $\mathbb{Z}_{3}$ on $A=\mathrm{C}^{*}\left(\mathbb{Z}_{9}\right)$, described by the morphism $\beta \in \operatorname{Mor}\left(A, A \otimes \mathrm{C}\left(\mathbb{Z}_{3}\right)\right)$ via the usual formula $\left(n \in \mathbb{Z}_{9}\right)$ :

$$
\beta\left(\lambda_{n}\right)=\lambda_{n} \otimes \delta_{\overline{0}}+\lambda_{\phi(n)} \otimes \delta_{\overline{1}}+\lambda_{\phi^{2}(n)} \otimes \delta_{\overline{2}}
$$

It is easy to verify that $\beta$ preserves the trace $\tau$ (so that Proposition 6.13 applies), and at the same time considering say $n=1$ we see that $\beta$ does not preserve the individual subspaces in the filtration we defined on $\mathrm{C}^{*}\left(\mathbb{Z}_{9}\right)$.

Proposition 6.15. Consider the orthogonal filtration $\widetilde{A} \rtimes_{\beta} \widetilde{B}$ on the algebra $\mathrm{C}^{*}\left(\mathbb{Z}_{9}\right) \rtimes_{\beta}$ $\mathbb{Z}_{3}$ defined by the family $\left\{A_{i j}: i, j \geq 0\right\}$ (as in Proposition 6.13). Then $\operatorname{QISO}\left(\widetilde{A} \rtimes_{\beta}\right.$ $\widetilde{B})$ is not isomorphic to the generalized Drinfeld's double of $\operatorname{QISO}(\widetilde{A})$ and $\operatorname{QISO}(\widetilde{B})$ with respect to any bicharacter. In particular there is no Hopf ${ }^{*}$-homomorphism $\pi \in$ $\operatorname{Mor}\left(\mathrm{C}\left(\mathrm{QISO}(\widetilde{A}), \mathrm{C}\left(\mathbb{Z}_{3}\right)\right)\right.$ such that $(\mathrm{id} \otimes \pi) \circ \gamma_{A}^{\mathrm{u}}=\beta$. 
Proof. As discussed before, the action $\beta$ comes from an action of $\mathbb{Z}_{3}$ on $\mathbb{Z}_{9}$ by automorphisms, which we denote by the same letter. We will use the identification $C^{*}\left(\mathbb{Z}_{9}\right) \rtimes_{\beta}$ $\mathbb{Z}_{3} \cong \mathrm{C}^{*}\left(\mathbb{Z}_{9} \rtimes_{\beta} \mathbb{Z}_{3}\right)$, under which the state $\tau$ can be identified with the canonical tracial state on $C^{*}\left(\mathbb{Z}_{9} \rtimes_{\beta} \mathbb{Z}_{3}\right)$. We can use the standard (symmetric) generating set to define the word-length function $l: \mathbb{Z}_{9} \rtimes_{\beta} \mathbb{Z}_{3} \rightarrow \mathbb{N}_{0}$ and for $n \geq 0$ put $U_{n}=\operatorname{Span}\left\{\lambda_{t}: t \in \mathbb{Z}_{9} \rtimes \mathbb{Z}_{3}, l(t)=n\right\}$. Then $\left(\mathrm{C}^{*}\left(\mathbb{Z}_{9} \rtimes_{\beta} \mathbb{Z}_{3}\right), \tau_{A},\left\{U_{n}\right\}_{n \geq 0}\right)$ is yet another orthogonal filtration of $\mathrm{C}^{*}\left(\mathbb{Z}_{9}\right) \rtimes_{\beta} \mathbb{Z}_{3}$. Moreover, $\left\{A_{i j}\right\}_{i, j \geq 0}$ is a sub-filtration of $\left\{U_{n}\right\}_{n \geq 0}$ in the sense of [8] so that by Corollary 2.11 of that paper, $\operatorname{QISO}\left(\widetilde{A} \rtimes_{\beta} \widetilde{B}\right)$ is a quantum subgroup of $\operatorname{QISO}\left(\mathrm{C}^{*}\left(\mathbb{Z}_{9} \rtimes_{\beta} \mathbb{Z}_{3}\right), \tau,\left\{U_{n}\right\}_{n \geq 0}\right)$. Now by the first computation in Section 5 of [31], $\mathrm{C}\left(\mathrm{QISO}\left(\mathrm{C}^{*}\left(\mathbb{Z}_{9} \rtimes_{\beta} \mathbb{Z}_{3}\right), \tau,\left\{U_{n}\right\}_{n \geq 0}\right)\right)$ is isomorphic to $\mathrm{C}^{*}\left(\mathbb{Z}_{9} \rtimes_{\beta} \mathbb{Z}_{3}\right) \oplus \mathrm{C}^{*}\left(\mathbb{Z}_{9} \rtimes_{\beta} \mathbb{Z}_{3}\right)$, so it has the vector space dimension equal $27+27=54$. Therefore, the vector space dimension of $\mathrm{C}\left(\mathrm{QISO}\left(\widetilde{A} \rtimes_{\beta} \widetilde{B}\right)\right)$ is no greater than 54 .

On the other hand the Hopf $C^{*}$-algebra of each generalized Drinfeld's double of $\operatorname{QISO}(\widetilde{A}))$ and $\mathrm{QISO}\left(\widetilde{\left.\mathrm{C}^{*}\left(\mathbb{Z}_{3}\right)\right)}\right.$ as a vector space is isomorphic to the tensor product of $\mathrm{C}\left(\mathrm{QISO}\left(\widehat{\mathrm{C}^{*}\left(\mathbb{Z}_{9}\right)}\right)\right)$ and $\mathrm{C}\left(\mathrm{QISO}\left(\widetilde{\mathrm{C}^{*}\left(\mathbb{Z}_{3}\right)}\right)\right)$. Since by Remark 4.7, $\mathrm{C}\left(\mathrm{QISO}\left(\widetilde{\mathrm{C}^{*}\left(\mathbb{Z}_{n}\right)}\right)\right) \cong$ $\mathrm{C}^{*}\left(\mathbb{Z}_{n}\right) \oplus \mathrm{C}^{*}\left(\mathbb{Z}_{n}\right)$ for $n \neq 4$, the vector space dimension of $\mathrm{C}\left(\mathrm{QISO}\left(\mathrm{C}^{*}\left(\mathbb{Z}_{9}\right)\right)\right) \otimes$ $\mathrm{C}\left(\mathrm{QISO}\left(\mathrm{C}^{*}\left(\mathbb{Z}_{3}\right)\right)\right)$ equals $(9+9)(3+3)=108$. This completes the proof of the main part of the proposition.

The last statement follows now from Theorem 6.2 (but can be also shown directly).

6.5. Further perspectives. Finally we outline two possible extensions of the results of previous subsections, namely, the cases of twisted crossed products and (twisted) crossed products by discrete quantum groups.

Let us recall the notion of twisted crossed products (see [11] and references therein). Let $\Gamma$ be a discrete group and $\Omega: \Gamma \times \Gamma \rightarrow S^{1}$ be a 2-cocycle on $\Gamma$, i.e, a map that satisfies the equation:

$$
\Omega(g, h) \Omega(g h, k)=\Omega(g, h k) \Omega(h, k), \quad g, h, k \in \Gamma .
$$

Let us then write $\tilde{\Omega}(h, k)=\Omega\left(k^{-1}, h^{-1}\right), h, k \in \Gamma$. For each $g \in \Gamma$ define $\lambda_{g}^{\Omega}$ and $\rho_{g}^{\tilde{\Omega}}$ to be the 'twisted' left and right shift operators on $\ell^{2}(\Gamma)$, given by

$$
\lambda_{g}^{\Omega}=\tilde{\Omega}\left(g^{-1}, \cdot\right) \lambda_{g}, \rho_{g}^{\tilde{\Omega}}=\tilde{\Omega}(\cdot, g) \rho_{g},
$$

where $\lambda_{g}$ and $\rho_{g}$ are the usual shift unitaries acting on $\ell^{2}(\Gamma)$. It follows that $\lambda_{g}^{\Omega} \lambda_{h}^{\Omega}=$ $\Omega(g, h) \lambda_{g h}^{\Omega}, \rho_{g}^{\tilde{\Omega}} \rho_{h}^{\tilde{\Omega}}=\tilde{\Omega}(g, h) \rho_{g h}^{\tilde{\Omega}}$ for any $g, h \in \Gamma$.

The twisted group $C^{*}$-algebra $C_{\mathrm{r}}^{*}(\Gamma, \Omega)$ is defined as the closed linear span of $\left\{\rho_{g}^{\tilde{\Omega}} \mid\right.$ $g \in \Gamma\}$ in $\mathbb{B}\left(\ell^{2}(\Gamma)\right)$. The $\mathrm{C}^{*}$-algebra $\mathrm{C}_{\mathrm{r}}^{*}(\Gamma, \Omega)$ is equipped with a canonical coaction of $\mathrm{C}_{\mathrm{r}}^{*}(\Gamma)$, i.e. the morphism $\delta \in \operatorname{Mor}\left(\mathrm{C}_{\mathrm{r}}^{*}(\Gamma, \Omega), \mathrm{C}_{\mathrm{r}}^{*}(\Gamma, \Omega) \otimes \mathrm{C}_{\mathrm{r}}^{*}(\Gamma)\right)$ given by the formula

$$
\delta\left(\rho_{g}^{\Omega}\right):=\sigma\left(\mathrm{W}^{*}\left(1 \otimes \rho_{g}^{\Omega}\right) \mathrm{W}\right)=\rho_{g}^{\Omega} \otimes \rho_{g}, \quad g \in \Gamma,
$$

where the operator $\mathrm{W} \in \mathcal{U}\left(\ell^{2}(\Gamma \times \Gamma)\right)$ is defined by $\mathrm{W} \xi(g, h)=\xi(g h, h)$ (with $g, h \in$ $\Gamma)$ and $\sigma$ is the usual flip. Now if $A$ is a unital $\mathrm{C}^{*}$-algebra and $\beta \in \operatorname{Mor}\left(A, A \otimes \mathrm{C}_{0}(\Gamma)\right)$ is a coaction, then the twisted crossed product $A \rtimes_{\beta, \mathrm{r}, \Omega} \Gamma$ is defined as the closed linear $\operatorname{span}$ of $\operatorname{Span}\left\{\beta(A)\left(1 \otimes \mathrm{C}_{\mathrm{r}}^{*}(\Gamma, \Omega)\right)\right\}$ in $\mathcal{M}\left(A \otimes \mathbb{K}\left(\ell^{2}(\Gamma)\right)\right)$. 
Following the same line of argument as that in the proof of Proposition 2.13, we obtain a $\mathrm{C}^{*}$-algebra isomorphism

$$
\Psi: A \nabla_{\mathrm{W}} \mathrm{C}_{\mathrm{r}}^{*}(\Gamma, \omega) \rightarrow A \rtimes_{\beta, \mathrm{r}, \Omega} \Gamma
$$

such that for all $a \in A$ and $g \in \Gamma$ we have

$$
\Psi\left(j_{A}(a)\right)=\beta(a), \Psi\left(j_{C_{\mathrm{r}}^{*}(\Gamma, \omega)}\left(\rho_{g}^{\Omega}\right)\right)=1 \otimes \rho_{g}^{\Omega} .
$$

Let $\widetilde{A}:=\left(A, \tau_{A},\left\{A_{i}\right\}_{i \geq 0}\right)$ denote an orthogonal filtration of a unital $\mathrm{C}^{*}$-algebra $A$. Let $\Gamma$ be a discrete group with a proper length function $l$. Define $B_{n}^{l, \Omega}=\operatorname{Span}\left\{\rho_{g}^{\tilde{\Omega}}\right.$ : $l(g)=n\}$. Then $\widetilde{B}:=\left(C_{\mathrm{r}}^{*}(\Gamma, \Omega), \tau_{\Gamma},\left\{B_{n}^{l, \Omega}\right\}_{n \in \mathbb{N}}\right)$ is an orthogonal filtration. Suppose $\beta$ satisfies (6.2). Then by the arguments analogous to those of Sect. 5 we can prove that $\operatorname{QISO}\left(\widetilde{A} \rtimes_{\beta} \widetilde{B}\right) \cong \mathfrak{D}_{\mathrm{V}}$, where $\mathfrak{D}_{\mathrm{V}}$ is the Drinfeld double of $\operatorname{QISO}(\widetilde{\widetilde{A}})$ and $\operatorname{QISO}(\widetilde{B})$ determined by $\mathrm{V}$.

Let us finish the article by mentioning that all the results of Sect. 6, and in particular Theorem 6.2, remain true if we consider actions of finitely generated discrete quantum groups (and length functions on such quantum groups) instead of classical discrete groups. Moreover, the results on twisted crossed products also go through for discrete quantum group actions.

Acknowledgements. The second author was supported by the National Postdoctoral Fellowship given by SERB-DST, Government of India Grant No. PDF/2017/001795. The third author was partially supported by an Early Career Research Award given by SERB-DST, Government of India Grant No. ECR/2017/001354. The last author was partially supported by the National Science Centre (NCN) Grant No. 2014/14/E/ST1/00525. This work was started during the internship of the second author at IMPAN in 2016, funded by the Warsaw Center for Mathematical Sciences. A.M. thanks A.S. for his kind hospitality at IMPAN. We thank the referees for their thoughtful comments and suggestions.

Open Access This article is distributed under the terms of the Creative Commons Attribution 4.0 International License (http://creativecommons.org/licenses/by/4.0/), which permits unrestricted use, distribution, and reproduction in any medium, provided you give appropriate credit to the original author(s) and the source, provide a link to the Creative Commons license, and indicate if changes were made.

\section{References}

1. Aubrun, G., Skalski, A., Speicher, R.: Quantum Symmetries, Lecture Notes in Mathematics, vol. 2189. Springer, Berlin (2017). http://dx.doi.org/10.1007/978-3-319-63206-3

2. Baaj, S., Skandalis, G.: Unitaires multiplicatifs et dualité pour les produits croisés de $C^{*}$-algèbres. Ann. Sci. École Norm. Sup. (4), 26(4), 425-488 (1993). http://www.numdam.org/item?id=ASENS_1993_4_ 26_4_425_0

3. Banica, T.: Quantum automorphism groups of homogeneous graphs. J. Funct. Anal. 224(2), 243280 (2005). http://dx.doi.org/10.1016/j.jfa.2004.11.002

4. Banica, T.: Quantum automorphism groups of small metric spaces. Pac. J. Math. 219(1), 27-51 (2005) http://dx.doi.org/10.2140/pjm.2005.219.27

5. Banica, T.: Quantum isometries, noncommutative spheres, and related integrals (2016). eprint, arXiv:1601.02159

6. Banica, T., Skalski, A.G.: Two-parameter families of quantum symmetry groups. J. Funct. Anal. 260(11), 3252-3282 (2011) http://dx.doi.org/10.1016/j.jfa.2010.11.016

7. Banica, T., Skalski, A.G.: Quantum isometry groups of duals of free powers of cyclic groups. Int. Math. Res. Not. IMRN 9, 2094-2122 (2012). http://dx.doi.org/10.1093/imrn/rnr098

8. Banica, T., Skalski, A.G.: Quantum symmetry groups of $C^{*}$-algebras equipped with orthogonal filtrations. Proc. Lond. Math. Soc. (3) 106(5), 980-1004 (2013) http://dx.doi.org/10.1112/plms/pds071

9. Bhowmick, J., Goswami, D.: Quantum group of orientation-preserving Riemannian isometries. J. Funct. Anal. 257(8), 2530-2572 (2009) http://dx.doi.org/10.1016/j.jfa.2009.07.006 
10. Bhowmick, J., Goswami, D., Skalski, A.: Quantum isometry groups of 0-dimensional manifolds. Trans. Am. Math. Soc. 363(2), 901-921 (2011) http://dx.doi.org/10.1090/S0002-9947-2010-05141-4

11. Bhowmick, J., Neshveyev, S., Sangha, A.: Deformation of operator algebras by Borel cocycles. J. Funct. Anal. 265(6), 983-1001 (2013) http://dx.doi.org/10.1016/j.jfa.2013.05.021

12. Bhowmick, J., Skalski, A.: Quantum isometry groups of noncommutative manifolds associated to group $C^{*}$-algebras. J. Geom. Phys. 60(10), 1474-1489 (2010) http://dx.doi.org/10.1016/j.geomphys.2010.05. 007

13. Bichon, J.: Quantum automorphism groups of finite graphs. Proc. Am. Math. Soc. 131(3), 665-673 (2003) http://dx.doi.org/10.1090/S0002-9939-02-06798-9

14. Christensen, E., Ivan, C.: Spectral triples for AF $C^{*}$-algebras and metrics on the Cantor set. J. Oper. Theory. 56(1), 17-46 (2006)

15. Chamseddine, A.H., Connes, A.: Noncommutative geometry as a framework for unification of all fundamental interactions including gravity. Part I. Fortschr. Phys. 58(6), 553-600 (2010) http://dx.doi.org/10. 1002/prop.201000069

16. Connes, A.: Noncommutative geometry. Academic Press, Inc., San Diego, CA (1994)

17. Cuntz, J., Krieger, W.: A class of $C^{*}$-algebras and topological Markov chains. Invent. Math. 56(3), 251268 (1980) http://dx.doi.org/10.1007/BF01390048

18. de Chanvalon, M.T.: Quantum symmetry groups of Hilbert modules equipped with orthogonal filtrations. J. Funct. Anal. 266(5), 3208-3235 (2014) http://dx.doi.org/10.1016/j.jfa.2013.10.020

19. Fischer, R.: Volle verschränkte Produkte für Quantengruppen und äquivariante KK-Theorie, Ph.D. Thesis, Westf. Wilhelms-Universität Münster (2003) http://nbn-resolving.de/urn:nbn:de:hbz:6-85659526538

20. Goswami, D.: Quantum group of isometries in classical and noncommutative geometry. Commun. Math. Phys. 285(1), 141-160 (2009) http://dx.doi.org/10.1007/s00220-008-0461-1

21. Goswami, D., Bhowmick, J.: Quantum Isometry Groups, Infosys Science Foundation Series, Infosys Science Foundation Series in Mathematical Sciences. Springer, New Delhi (2016). http://dx.doi.org/10. 1007/978-81-322-3667-2

22. Goswami, D., Roy, S.: Faithful actions of locally compact quantum groups on classical spaces. Lett. Math. Phys. 107(7), 1375-1390 (2017) http://dx.doi.org/10.1007/s11005-017-0951-1

23. Joardar, S., Mandal, A.: Quantum Symmetry of Graph C*-algebras associated with connected Graphs. eprint, arXiv:1711.04253 (2017)

24. Joardar, S., Mandal, A.: Quantum symmetry of graph $C^{*}$-algebras at critical inverse temperature. eprint, arXiv:1803.08012 (2018)

25. Kasprzak, P.: Rieffel deformation via crossed products. J. Funct. Anal. 257(5), 1288-1332 (2009) http:// dx.doi.org/10.1016/j.jfa.2009.05.013

26. Katsura, T.: The ideal structures of crossed products of Cuntz algebras by quasi-free actions of abelian groups. Can. J. Math. 55(6), 1302-1338 (2003) http://dx.doi.org/10.4153/CJM-2003-050-6

27. Kustermans, J.: Locally compact quantum groups in the universal setting. Int. J. Math. 12(3), 289338 (2001) http://dx.doi.org/10.1142/S0129167X01000757

28. Kustermans, J., Vaes, S.: Locally compact quantum groups. Ann. Sci. École Norm. Sup. (4) 33(6), 837934 (2000) http://dx.doi.org/10.1016/S0012-9593(00)01055-7

29. Kustermans, J., Vaes, S.: Locally compact quantum groups in the von Neumann algebraic setting. Math. Scand. 92(1), 68-92 (2003) http://dx.doi.org/10.7146/math.scand.a-14394

30. Kyed, D., Sołtan, P.M.: Property (T) and exotic quantum group norms. J. Noncommut. Geom. 6(4), 773800 (2012) http://dx.doi.org/10.4171/JNCG/105

31. Mandal, A.: Quantum isometry group of dual of finitely generated discrete groups-II. Ann. Math. Blaise Pascal 23(2), 219-247 (2016)

32. Masuda, T., Nakagami, Y.: A von Neumann algebra framework for the duality of the quantum groups. Publ. Res. Inst. Math. Sci 30(5), 799-850 (1994) http://dx.doi.org/10.2977/prims/1195165585

33. Masuda, T., Nakagami, Y., Woronowicz, S.L.: A $C^{*}$-algebraic framework for quantum groups. Int. J. Math. 14(9), 903-1001 (2003) http://dx.doi.org/10.1142/S0129167X03002071

34. Meyer, R., Roy, S., Woronowicz, S.L.: Homomorphisms of quantum groups. Münster J. Math. 5, 124 (2012) http://nbn-resolving.de/urn:nbn:de:hbz:6-88399662599

35. Meyer, R., Roy, S., Woronowicz, S.L.: Quantum group-twisted tensor products of $\mathrm{C}^{*}$-algebras. Int. J. Math. 25(2), 1450019-1450037 (2012) http://dx.doi.org/10.1142/S0129167X14500190

36. Pedersen, G.K.: $C^{*}$-algebras and their automorphism groups, London Mathematical Society Monographs, vol. 14, Academic Press, London (1979)

37. Raum, S., Weber, M.: A connection between easy quantum groups, varieties of groups and reflection groups. eprint, arXiv:1212.4742v2 (2017)

38. Rieffel, M.A.: Deformation quantization for actions of $\mathbf{R}^{d}$. Mem. Am. Math. Soc. 106(506):x+93 (1993) http://dx.doi.org/10.1090/memo/0506

39. Roy, S.: The Drinfeld double for $C^{*}$-algebraic quantum groups. J. Oper. Theory 74(2), 485-515 (2015) http://dx.doi.org/10.7900/jot.2014sep04.2053 
40. Roy, S., Timmermann, T.: The maximal quantum group-twisted tensor product of $C^{*}$-algebras. J. Noncommut. Geom. 12(1), 279-330 (2018) http://dx.doi.org/10.4171/JNCG/277

41. Schmidt, S., Weber, M.: Quantum symmetries of graph $C^{*}$-algebras. eprint, arXiv:1706.08833v2 (2017)

42. Sołtan, P.M., Woronowicz, S.L.: From multiplicative unitaries to quantum groups. II. J. Funct. Anal. 252(1), 42-67 (2007) http://dx.doi.org/10.1016/j.jfa.2007.07.006

43. Speicher, R., Weber, M.: Quantum groups with partial commutation relations. eprint, arXiv:1603.09192v1 (2017)

44. Van Daele, A.: An algebraic framework for group duality. Adv. Math. 140(2), 323-366 (1998) http://dx. doi.org/10.1006/aima.1998.1775

45. Drinfel'd, V.: Quantum groups. In: Proceedings of the International Congress of Mathematicians, vols. 1, 2, Berkeley, CA, pp. 798-820 (1987)

46. Wang, S.: Quantum symmetry groups of finite spaces. Commun. Math. Phys. 195(1), 195-211 (1998) http://dx.doi.org/10.1007/s002200050385

47. Woronowicz, S.L.: From multiplicative unitaries to quantum groups. Int. J. Math. 7(1), 127-149 (1996) http://dx.doi.org/10.1142/S0129167X96000086

48. Woronowicz, S.L.: Compact quantum groups, Symétries quantiques, Les Houches, 1995, vol. 1998. North-Holland, Amsterdam, pp. 845-884 (1995)

Communicated by Y. Kawahigashi 